\title{
Magnetic and Transport Studies of Strongly Correlated Perovskite Ceramics
}

\author{
by \\ Erwan Hemery
}

\author{
A thesis \\ submitted to the Victoria University of Wellington \\ in fulfilment of the \\ requirements for the degree of \\ Doctor of Philosophy \\ in Physics. \\ Victoria University of Wellington \\ 2007
}




\begin{abstract}
This thesis describes the results from an experimental study of the magnetic and transport properties of two strongly correlated transition metal oxides. The first material under study is the ferromagnetic half-metal double perovskite, $\mathrm{Sr}_{2} \mathrm{FeMoO}_{6}$, in which we have made isoelectronic $\left(\mathrm{Ba}^{2+}\right)$ and electronic $\left(\mathrm{La}^{3+}\right)$ substitutions onto the strontium site. Magnetoresistance measurements on $\mathrm{Sr}_{2-x} \mathrm{Ba}_{x} \mathrm{FeMoO}_{6}$ revealed that the low temperature magnetoresistance is dominated by inter-grain transport while the intra-grain contribution is evident when the temperature is close to the ferromagnetic transition temperature. Transport measurements on

$\mathrm{Sr}_{2-x} \mathrm{La}_{x} \mathrm{FeMoO}_{6}$ clearly showed that the doping dependence of the thermoelectric power is surprisingly similar to the one observed in the superconducting cuprates. In addition, it was found that the electronic doping leads to an increase in the ferromagnetic transition temperature, which supports the band filling model. Substitution on the Fe site was also investigated by partially replacing Fe with the non-magnetic aluminium element $\left(\mathrm{Sr}_{2} \mathrm{Fe}_{1-x} \mathrm{Al}_{x} \mathrm{MoO}_{6}\right)$. It was found from thermoelectric power measurement that the Fe electronic state is below 3+, which is inconsistent with theoretical models but is in good agreement with Mössbauer measurements. In addition, magnetic measurements showed that the reduction in the ferromagnetic ordering temperature could be explained in terms of a 3D percolation model.

The second compound is the oxygen deficient strontium iron oxide $\mathrm{SrFeO}_{3-\delta}$. The temperature dependence of the thermoelectric power was measured in this compound for the first time and shown to be reminiscent of the charge-ordering Verwey transition observed in $\mathrm{Fe}_{3} \mathrm{O}_{4}$. Magnetic
\end{abstract}


measurements show an increase of a weak ferromagnetic signal versus the oxygen deficiency that could originate from a Dzyaloshinsky-Moriya interaction in the distorted $\mathrm{FeO}_{6}$ octahedra. Finally, we observed a large magnetoresistance near room temperature for compounds close to the orthorhombic $\mathrm{SrFeO}_{2.75}$ phase. 


\section{Acknowledgments}

I would like to express my sincere gratitude to my two supervisors Em. Prof. Joe Trodahl and Dr. Grant Williams their support and guidance throughout my PhD. I also would like to thank the staff of the Material and Communication group of Industrial Research Ltd for their technical assistance during my project.

I am particulary grateful to R. Gaál and L. Forró for the provision of the transport facility at the École Polytechnique Fédérale de Lausanne (EPLF) in Lausanne, Switzerland.

I also acknowledge funding support from the New Zealand Marsden Fund, MoRST, the MacDiarmid Institute, New Zealand Postgraduate Study Abroad Awards (NZPSAA) and the Victoria University science faculty research grant.

Finally, I would like to thank Laurie, my sisters, parents and friends for their support throughout all my studies. 


\section{Contents}

1 Introduction $\quad 1$

2 Background 4

$2.1 \mathrm{Sr}_{2} \mathrm{FeMoO}_{6}$ and related double perovskites . . . . . . . . 4

2.2 Strontium iron oxide, $\mathrm{SrFeO}_{3-\delta} \ldots \ldots \ldots$. . . . . . . 10

3 Experimental details $\quad 14$

3.1 Sample preparation . . . . . . . . . . . . . . . . 14

3.1.1 Double perovskites . . . . . . . . . . . . 14

$3.1 .2 \mathrm{SrFeO}_{3-\delta} \ldots \ldots \ldots \ldots \ldots \ldots \ldots$

3.2 X-Ray Diffraction $(\mathrm{XRD}) \ldots \ldots \ldots \ldots$

3.3 Scanning Electron Microscope (SEM) . . . . . . . . . . . . 18

3.4 Thermoelectric Power (TEP) . . . . . . . . . . . . . . . 20

3.5 Temperature dependence of the resistivity $(\rho) \ldots \ldots 22$

3.6 Magnetoresistance $(\mathrm{MR}) \ldots \ldots . \ldots . \ldots 23$

3.6.1 Low field magnetoresistance . . . . . . . . . . 23

3.6.2 High field magnetoresistance up to $12 \mathrm{~T} \ldots \ldots . . .26$

3.7 Superconducting QUantum Interference Device Magnetome-

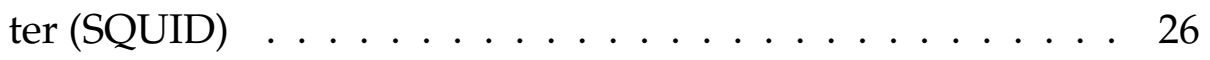

4 Isoelectronic substitution in $\mathrm{Sr}_{2-x} \mathrm{Ba}_{x} \mathrm{FeMoO}_{6}$

4.1 Structural characterisation . . . . . . . . . . . . 30

4.1.1 X-ray diffraction and degree of antisite disorder . . . 30 
4.1.2 Scanning electron microscope images . . . . . . . . 32

4.2 Magnetisation measurements . . . . . . . . . . . . . 34

4.2.1 Temperature and field dependence . . . . . . . . . . 34

4.2.2 Magnetoresistance measurements . . . . . . . . . 36

4.3 Summary . . . . . . . . . . . . . . . . . 45

5 Electron doping by lanthanum in $\mathrm{Sr}_{2-x} \mathrm{La}_{x} \mathrm{FeMoO}_{6} \quad 46$

5.1 X-ray diffraction patterns . . . . . . . . . . . 47

5.2 Transport properties in La-doped double perovskite . . . . . 48

5.2.1 Temperature dependence of the resistivity . . . . . . 48

5.2.2 Thermoelectric power of $\mathrm{Sr}_{2-x} \mathrm{La}_{x} \mathrm{FeMoO}_{6}$. . . . . . 50

5.3 Magnetisation measurements . . . . . . . . . . . . 54

5.3.1 Antisite disorder effect on the saturation magnetic moment . . . . . . . . . . . . . 54

5.3.2 Shift of the Curie temperature upon La-doping . . . . 56

5.4 Magnetoresistance in La-doped samples . . . . . . . . . . 57

5.5 summary . . . . . . . . . . . . . . . . . . 59

6 Magnetic and electronic properties of $\mathrm{Sr}_{2} \mathrm{Fe}_{1-x} \mathrm{Al}_{x} \mathrm{MoO}_{6} \quad 60$

6.1 Single phase materials $\mathrm{Sr}_{2} \mathrm{Fe}_{1-x} \mathrm{Al}_{x} \mathrm{MoO}_{6} \ldots \ldots$. . . . . . 62

6.2 Transport properties of $\mathrm{Sr}_{2} \mathrm{Fe}_{1-x} \mathrm{Al}_{x} \mathrm{MoO}_{6} \ldots \ldots \ldots$

6.2.1 Resistivity measurements . . . . . . . . . . . 63

6.2.2 Thermoelectric power . . . . . . . . . . . . 64

6.3 Magnetic response of $\mathrm{Sr}_{2} \mathrm{Fe}_{1-x} \mathrm{Al}_{x} \mathrm{MoO}_{6} \ldots \ldots$. . . . . . . 65

6.3.1 Temperature and field dependence of the magnetic moment ................. 65

6.3 .2 Magnetoresistance . . . . . . . . . . . 70

6.3.3 Zero Field Nuclear Magnetic Resonance (ZFNMR) 72

6.4 Summary . . . . . . . . . . . . . . . . . 76

7 Magnetic and transport properties of oxygen deficient $\mathrm{SrFeO}_{3-\delta} \quad 77$

$7.1 \quad$ X-ray diffraction of $\mathrm{SrFeO}_{3-\delta} \ldots \ldots \ldots 78$ 
7.2 Magnetic response . . . . . . . . . . . . . . . . . 79

7.2.1 Determination of $\delta$ from the magnetic measurements $\quad 79$

7.2.2 Magnetic measurements . . . . . . . . . . . . . 81

7.3 Temperature dependence of the resistivity . . . . . . . . 84

7.3.1 Zero field resistivity measurements . . . . . . . . 84

7.3.2 Magneto-transport behaviour of $\mathrm{SrFeO}_{3-\delta} \ldots \ldots$. . . 86

7.4 Thermoelectric power measurements . . . . . . . . . . . . 89

7.4.1 Anomalous temperature dependence of the thermoelectric power . . . . . . . . . . . . . . . 89

7.4.2 Room temperature thermoelectric power . . . . . . 95

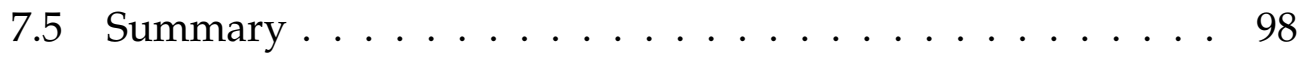

$\begin{array}{lr}\text { Summary and conclusions } & 99\end{array}$

$\begin{array}{ll}\text { References } & 104\end{array}$

$\begin{array}{ll}\text { List of Publications } & 113\end{array}$ 


\section{List of Figures}

$2.1 \mathrm{Sr}_{2} \mathrm{FeMoO}_{6}$ double perovskite lattice structure . . . . . . . 5

2.2 MR and magnetisation data from Kobayashi et al. . . . . . . 9

$2.3 \mathrm{AFeO}_{3}$ perovskite structure . . . . . . . . . . . . 11

$2.4 \mathrm{SrFeO}_{3}, \mathrm{Sr}_{4} \mathrm{Fe}_{4} \mathrm{O}_{11}$ and $\mathrm{Sr}_{8} \mathrm{Fe}_{8} \mathrm{O}_{23}$ crystal structures . . . . . . 13

3.1 X-ray diffraction pattern of $\mathrm{SrMoO}_{4}$ and $\mathrm{SrFeO}_{3-\delta} \ldots \ldots$. . . 15

3.2 Scanning electron microscope image of a $\mathrm{Ba}_{2} \mathrm{FeMoO}_{6}$ sample 19

3.3 Thermoelectric power apparatus . . . . . . . . . . . 21

3.4 Magnetoresistance apparatus . . . . . . . . . . . . 25

3.5 Configuration of the MPMS from Quantum design . . . . . . 27

4.1 XRD patterns of $\mathrm{Sr}_{2-x} \mathrm{Ba}_{x} \mathrm{FeMoO}_{6} \ldots \ldots \ldots$. . . . . . . 31

4.2 Scanning electron microscope images of $\mathrm{Sr}_{2-x} \mathrm{Ba}_{x} \mathrm{FeMoO}_{6}$. . 33

4.3 Magnetic moment versus the applied magnetic field at $10 \mathrm{~K}$ for the $\mathrm{Sr}_{2-x} \mathrm{Ba}_{x} \mathrm{FeMoO}_{6}$ samples . . . . . . . . . . . . 34

4.4 Magnetic moment versus temperature at $0.01 \mathrm{~T}$. . . . . . . 35

4.5 Magnetoresistance at $77 \mathrm{~K}_{\text {of }} \mathrm{Sr}_{2-x} \mathrm{Ba}_{x} \mathrm{FeMoO}_{6} \ldots \ldots$. . . . . 37

4.6 Magnetoresistance at $77 \mathrm{~K}$ and $300 \mathrm{~K}$. . . . . . . . . . . . 40

4.7 Magnetoresistance versus the resistivity at $0.8 \mathrm{~T}$ and $77 \mathrm{~K}$. . 41

4.8 Magnetoresistance at $4.2 \mathrm{~K}$ and $300 \mathrm{~K} \ldots . . . . . . . .44$

4.9 Plot of the MR versus the applied magnetic field . . . . . . . 44

5.1 XRD patterns of $\mathrm{Sr}_{2-x} \mathrm{La}_{x} \mathrm{FeMoO}_{6} \ldots \ldots \ldots$. . . . . . . 48

5.2 Temperature dependence of the resistivity . . . . . . . . . 49 
5.3 Thermoelectric power of $\mathrm{Sr}_{2-x} \mathrm{La}_{x} \mathrm{FeMoO}_{6}$. . . . . . . . . . 51

5.4 Magnetic moment versus applied magnetic field at $10 \mathrm{~K}$. . 55

5.5 Magnetic moment versus temperature of $\mathrm{Sr}_{2-x} \mathrm{La}_{x} \mathrm{FeMoO}_{6} \quad$. 57

5.6 Magnetoresistance at $77 \mathrm{~K}$ for $\mathrm{Sr}_{2-x} \mathrm{La}_{x} \mathrm{FeMoO}_{6} \ldots \ldots$. . . . 58

6.1 XRD patterns of $\mathrm{Sr}_{2} \mathrm{Fe}_{1-x} \mathrm{Al}_{x} \mathrm{MoO}_{6} \ldots \ldots \ldots$

6.2 Resistivity for $\mathrm{Sr}_{2} \mathrm{Fe}_{1-x} \mathrm{Al}_{x} \mathrm{MoO}_{6} \ldots \ldots \ldots$. . . . . . . 63

6.3 Thermoelectric power of $\mathrm{Sr}_{2} \mathrm{Fe}_{1-x} \mathrm{Al}_{x} \mathrm{MoO}_{6} \ldots \ldots$. . . . . . 65

6.4 Temperature and field dependence of $\mathrm{Sr}_{2} \mathrm{Fe}_{1-x} \mathrm{Al}_{x} \mathrm{MoO}_{6} \ldots 66$

6.5 Plot of $T_{c}$ against the $\mathrm{Al}$ and $\mathrm{Mn}$ fraction . . . . . . . . . 68

6.6 Magnetoresistance at $77 \mathrm{~K}$ and $300 \mathrm{~K}$ for $\mathrm{Sr}_{2} \mathrm{Fe}_{1-x} \mathrm{Al}_{x} \mathrm{MoO}_{6} \quad 71$

6.7 Plot of the ZFNMR spectra for $\mathrm{Sr}_{2} \mathrm{Fe}_{1-x} \mathrm{Al}_{x} \mathrm{MoO}_{6} \ldots$. . . . . 73

6.8 Plots from the ZFNMR analysis of $\mathrm{Sr}_{2} \mathrm{Fe}_{1-x} \mathrm{Al}_{x} \mathrm{MoO}_{6} \quad \ldots$. . 75

7.1 XRD patterns of the $\mathrm{SrFeO}_{2.95}$ and $\mathrm{SrFeO}_{2.72}$ compounds . . 79

$7.2 \mathrm{ZFC}$ and FC magnetisation at $1 \mathrm{~T} \ldots \ldots$. . . . . . . 80

$7.3 \mathrm{ZFC}$ and FC magnetisation at $6 \mathrm{~T} \ldots \ldots . \ldots 81$

7.4 Temperature dependence of the ac magnetisation . . . . . 83

7.5 FC measurements at $25 \mathrm{mT} \ldots \ldots$. . . . . . . . . 84

7.6 Temperature dependence of the resistivity . . . . . . . . 85

7.7 Temperature dependence of the resistivity at 0 and $12 \mathrm{~T} \ldots 87$

7.8 TEP versus temperature of $\mathrm{SrFeO}_{3-\delta} \ldots \ldots \ldots$. . . . . . 90

7.9 Fitting curves of the TEP versus temperature of $\mathrm{SrFeO}_{3-\delta} \ldots 93$

7.10 Plot of $\Delta S$ against $3-\delta \ldots \ldots \ldots \ldots \ldots$

7.11 Thermoelectric power at room temperature . . . . . . . . 96 


\section{Chapter 1}

\section{Introduction}

The independent electron approximation model describes the properties of most common solid state physics materials in which the repulsion between the electrons can be represented by using an average effective potential [1]. However, in some narrow band systems and where the concentration of the conduction electrons is low, this approximation is no longer valid as the Coulomb interaction between the electrons becomes important; the materials are then referred to as strongly correlated electron systems. Due to the highly correlated nature of the electrons, the materials exhibit complex transport and magnetic properties (e.g. high temperature superconductivity, half-metallicity, etc) and for this reason they have been for many years one of the major research topics in solid state physics.

The most interesting Transition Metal Oxides (TMO) belong to this class of material in which this interaction occurs within the $d$-electrons and in which there is a strong Coulomb repulsion. A direct effect of this strong correlation is the interplay between the electronic, spin and lattice degrees of freedom. This coupling is subtle and therefore complex to study; for example a small change in the chemical composition such as oxygen deficiency or trivalent substitution on a divalent site can lead to drastic changes in the properties.

The high temperature superconducting copper oxide is a TMO and was 
discovered twenty years ago and has become one of the most studied materials in solid state physics. Before the discovery of Bednorz and Müller in 1986 of "high temperature" superconductivity in the $\mathrm{La}_{2-x} \mathrm{Ba}_{x} \mathrm{CuO}_{4}$ perovskite $[2,3]$, the highest known superconducting transition was $\mathrm{Nb}_{3} \mathrm{Ge}$ with a transition temperature of $T_{c}=24 \mathrm{~K}$. Since then, many investigators have synthesised these copper oxide based materials worldwide including the well-know "1:2:3" $\mathrm{YBa}_{2} \mathrm{Cu}_{3} \mathrm{O}_{7-\delta}$ being the first solid showing superconductivity above liquid nitrogen temperature $\left(T_{c}=95 \mathrm{~K}\right)$. The quest to find the highest transition temperature is still on with the $\mathrm{Hg}$-based cuprates leading at $T_{c}=164 \mathrm{~K}$ under high pressure [4].

Other interesting materials in the transition metal oxide family are the manganites, having a single perovskite structure of the $\mathrm{AMnO}_{3}$ form. In the perovskite manganite, the $d$-levels split into $\mathrm{t}_{2 g}$ and $\mathrm{e}_{g}$ orbitals caused by the surrounding oxygen anions. The electronic ground state with orbital degeneracy will distort to remove the degeneracy; this is known as the Jahn-Teller distortion. In addition, these compounds are well-known for their large change in resistance with applied magnetic field [5, 6] known as Magnetoresistance (MR).

In recent years, magnetoresistance phenomena have been extensively studied and in particular spin-polarised tunneling magnetoresistance since its discovery at room temperature in ferromagnetic metal tunnel junctions $[7,8]$. Spin-polarised tunneling provides fascinating opportunities not only for the understanding of fundamental aspects but also for application purposes. The spin tunneling junction is the basic of spintronics (spin transport electronics), which also uses the electron spin instead of only the charge of the electron as in the standard semiconductor industry. The tunneling magnetoresistance depends critically on the degree of spin polarisation, $P$, which can be written as $P=(\mathrm{n} \downarrow-\mathrm{n} \uparrow) /(\mathrm{n} \downarrow+\mathrm{n} \uparrow)$, where $\mathrm{n} \downarrow(\mathrm{n} \uparrow)$ is the fraction of carriers in the majority(minority) spin state at the Fermi energy. A fully spin polarised material $(P=1)$ at room temperature is desirable to enhance the magnetoresistance in view of new de- 
vices $[9,10]$.

This thesis presents the results from an experimental study of two strongly correlated electron oxides: the ferromagnetic half-metal double perovskite $\mathrm{Sr}_{2} \mathrm{FeMoO}_{6}$ which has a large negative magnetoresistance at room temperature and the strontium iron oxide perovskite material $\left(\mathrm{SrFeO}_{3-\delta}\right)$ which exhibits unconventional electronic and magnetic properties that change with the oxygen deficiency. Chapter 2 contains a review of these two systems including their main properties and pointing out some of the unsolved questions. Chapter 3 gives a description of the sample preparation and experimental methods used for the characterisation of the materials. Chapter 4 summarizes the experimental results obtained upon an isoelectronic substitution $\left(\mathrm{Ba}^{2+}\right)$ on the strontium site in $\mathrm{Sr}_{2} \mathrm{FeMoO}_{6}$ and focuses on the magnetoresistance measurements. Chapter 5 reports measurements on electron-doped samples $\left(\mathrm{La}^{3+}\right.$ substitution on the $\mathrm{Sr}^{2+}$ site) and is centred on the thermoelectric power results. Chapter 6 consists of the magnetic and electronic transport measurements from a series of aluminium substituted compounds, $\mathrm{Sr}_{2} \mathrm{Fe}_{1-x} \mathrm{Al}_{x} \mathrm{MoO}_{6}$. And finally chapter 7 focuses on the electronic and magnetic properties of the oxygen deficient $\mathrm{SrFeO}_{3-\delta}$ perovskite. Temperature dependence of the thermoelectric power and magnetic measurements have been carried out to provide further insight into the unusual properties of $\mathrm{SrFeO}_{3-\delta}$. 


\section{Chapter 2}

\section{Background}

In this chapter, we introduce the two materials which were studied in this thesis namely the double perovskites and the strontium iron oxides. In addition, throughout this chapter, some of the remaining questions about these strongly correlated electron systems are pointed out.

\section{1 $\mathrm{Sr}_{2} \mathrm{FeMoO}_{6}$ and related double perovskites}

The ordered double perovskite $\mathrm{A}_{2} \mathrm{BB}^{\prime} \mathrm{O}_{6}$ is composed of a rare-earth ion (A) and two $\mathrm{BO}_{6}$ and $\mathrm{B}^{\prime} \mathrm{O}_{6}$ octahedra placed alternatively in the $3 \mathrm{D}$ crystal lattice. The most studied double perovskite is $\mathrm{Sr}_{2} \mathrm{FeMoO}_{6}$ (SFMO) with an assumed $\mathrm{Fe}^{3+}$ (spin quantum number $\left.\mathrm{s}=5 / 2\right)$ and $\mathrm{Mo}^{5+}(\mathrm{s}=1 / 2)($ see figure 2.1).

The $\mathrm{Fe}^{3+} 3 \mathrm{~d}^{5}$ up-spin electrons can be considered to be localised while the $\mathrm{Mo}^{5+} 4 \mathrm{~d}^{1}$ down-spin electrons are delocalised and contribute to the itinerant electron transport. It has been argued from band structure calculations that the up-spins are localised in the $\mathrm{Fe}\left(\mathrm{t}_{2 g}\right)$ and $\left(\mathrm{e}_{g}\right)$ orbitals and the conduction band has $\mathrm{Mo}\left(\mathrm{t}_{2 g}\right)$ and $\mathrm{Fe}\left(\mathrm{t}_{2 g}\right)$ character and contains only down-spins [11, 12]. The results predict that there is a gap of $\sim 0.8 \mathrm{eV}$ in the up-spin Density Of States (DOS) across the Fermi energy, $E_{F}$, while a finite DOS is present for the down-spin. 


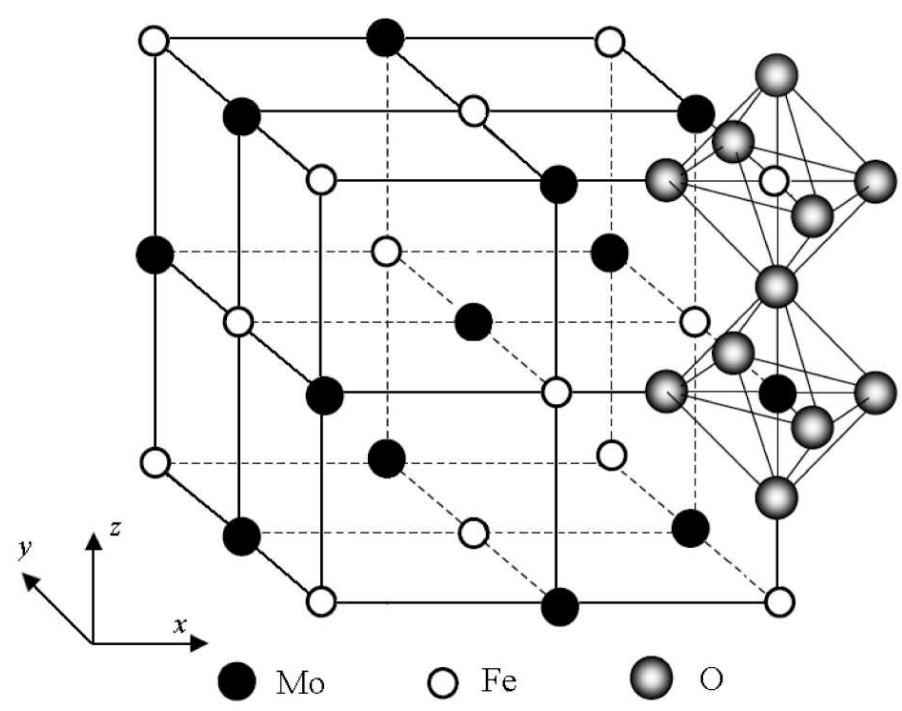

Figure 2.1: Sketch of the $\mathrm{Sr}_{2} \mathrm{FeMoO}_{6}$ double perovskite lattice structure. The strontium element is in the centre of each of the cells but is not shown for clarity.

There is debate concerning the exchange interaction that leads to the ferromagnetic state. It has been argued that double exchange occurs in SFMO, because the Curie temperature $\left(T_{c}\right)$ increases with increasing itinerant charge density and width of the conduction band [13,14] via partial substitution of $\mathrm{Sr}^{2+}$ by $\mathrm{La}^{3+}$ [15]. A similar increase in the Curie temperature was found in $\mathrm{Ca}_{2-x} \mathrm{Nd}_{x} \mathrm{FeMoO}_{6}$ where $\mathrm{Nd}^{3+}$ is partially substituted onto the $\mathrm{Ca}^{2+}$ site [16]. The double exchange interaction was first used to explain the ferromagnetic properties of $\mathrm{Fe}_{3} \mathrm{O}_{4}$ and the manganite perovskite system. In the latter, the alternate mixed valence of $\mathrm{Mn}^{3+}$ and $\mathrm{Mn}^{4+}$ separated by oxygen gives rise to a large conductivity and a ferromagnetic interaction. The localised $\left(\mathrm{t}_{2 g}\right)$ and delocalised $\left(\mathrm{e}_{g}\right)$ electrons are coupled ferromagnetically by a strong on-site Hund's coupling. In the double perovskite, the situation seems different, the $\mathrm{Fe}\left(\mathrm{t}_{2 g}\right) \downarrow$ and the $\mathrm{Mo}\left(\mathrm{t}_{2 g}\right) \downarrow$ bands are empty and the electron can hop between these states. 
The Fe $3 \mathrm{~d} \uparrow$ are full and therefore can accept only an antiparallel itinerant electron from the $\operatorname{Mo}\left(t_{2 g}\right) \downarrow$ and not the up-spin [17, 18]. Sarma et al. $[19,20]$ argued that double exchange does not occur in SFMO because the Fe $3 \mathrm{~d}^{5}$ up-spin orbitals are full which pushes the down-spin electron to be delocalised. Therefore, Hund's coupling cannot occur between the itinerant down-spin Mo $4 \mathrm{~d}^{1}$ electron and the up-spin localised $3 \mathrm{~d}^{5}$ electrons on the Fe site. Even though the analogy with the double exchange was tempting, the ferromagnetism in the double perovskite seems to originate from a different mechanism.

The ferromagnetic superexchange coupling between the B-site in B-OB'-O-B (e.g. Fe-O-Mo-O-Fe) has also been proposed to explain the magnetic properties but this would assume an unoccupied state on the B' site as observed by neutron diffraction measurement [21]. This coupling could explain the low Néel temperature transition $\left(T_{N} \backsim 20-40 \mathrm{~K}\right)$ and insulating behaviour observed in some double perovskites such as $\mathrm{Sr}_{2} \mathrm{FeWO}_{6}$ [22]. However, this model contradicted the temperature dependent transport data that show metallic behaviour in the $\mathrm{Sr}_{2} \mathrm{FeMoO}_{6}$ compound. In addition, the high $T_{c}$ value observed in $\mathrm{Sr}_{2} \mathrm{FeMoO}_{6}$ would not be expected from weak ferromagnetic coupling.

This leads to another model in which the Fe 3d - Mo 4d hybridisation and an exchange splitting of the Mo orbitals result in antiferromagnetic coupling between the localised Fe $3 \mathrm{~d}^{5}$ up-spins and the itinerant Mo $4 \mathrm{~d}^{1}$ down-spin $[19,20]$. It is assumed that there is significant hopping between Mo and Fe that leads to an admixture of down-spin Fe( $\left.t_{2 g}\right)$ and $\mathrm{Mo}\left(\mathrm{t}_{2 g}\right)$ orbitals. Due to the hybridisation with the Fe states the Fe(t $\left.t_{2 g}\right) \downarrow$ and $\mathrm{Mo}\left(\mathrm{t}_{2 g}\right)$ $\downarrow$ orbitals are pushed down while the $\mathrm{Fe}\left(\mathrm{t}_{2 g}\right) \uparrow$ and $\mathrm{Mo}\left(\mathrm{t}_{2 g}\right) \uparrow$ orbitals go up. The net result is that the Fermi level is contained within a band that has down-spin $\mathrm{Fe}\left(\mathrm{t}_{2 g}\right)$ and $\mathrm{Mo}\left(\mathrm{t}_{2 g}\right)$ character and produces a spin polarisation of the conduction electrons [12]. We are therefore in the case of an antiferromagnetic coupling between the itinerant Mo $4 \mathrm{~d}^{1}$ and the localised $3 \mathrm{~d}^{5}$ electrons [23]. Despite the extensive amount of work performed on the 
double perovskites there is still an open debate about the exchange mechanism leading to the exotic magnetic and electronic transport properties.

Magnetisation data at low temperature and high field show a magnetic moment of around 3 - $3.5 \mu_{B} / f . u$. (Bohr magnetons per formula unit), which is smaller than the $4 \mu_{B} / f . u$. predicted from the simple antiferromagnetic coupling model with the $\mathrm{Fe}^{3+}\left(3 \mathrm{~d}^{5} ; \mathrm{t}^{3}{ }_{2 g} \uparrow \mathrm{e}_{g}{ }^{2} \uparrow\right)-\mathrm{Mo}^{5+}\left(4 \mathrm{~d}^{1}\right.$; $\mathbf{t}^{1}{ }_{2 g} \downarrow$ ) arrangement. It is believed that the Anti-Site Disorder (ASD) is the cause of the discrepancy from the perfect saturation magnetic moment of $4 \mu_{B} / f . u$. In the ideal picture, the Fe and Mo are alternatively placed in the structure surrounded by oxygen. However, some disorder between the two cations can occur, which means that two Fe atoms or Mo can sit next to each other [24]. It can be easily understood that ASD affects the transport and magnetic properties as the orbitals overlapping would be altered upon the degree of ASD. For example, the degree of antisite disorder plays a role on the Curie temperature [25] and the magnetoresistance [26]. However, the exact effect of the antisite disorder on the double perovskite properties is still a dilemma that needs further investigation.

Another interesting feature of this compound is the high critical temperature, Curie temperature $\left(T_{c}\right)$ for a ferromagnet, of the order of $400 \mathrm{~K}$ [11]. According to the mean field theory [27], the Curie temperature is directly correlated with inter-atomic exchange coupling between the two cations, which in the case of the $\mathrm{Sr}_{2} \mathrm{FeMoO}_{6}$ double perovskite is of the order of $40 \mathrm{meV}$ [24]. However, the mean field approach is a poor approximation and investigators are still looking for answers by varying $T_{c}$ using atomic substitutions.

Interestingly, there are also some controversial results regarding the electronic states of the $\mathrm{Fe}$ and $\mathrm{Mo}$ in $\mathrm{Sr}_{2} \mathrm{FeMoO}_{6}$. X-ray absorption and photoemission spectroscopy have shown that the ground state is close to $\mathrm{Fe}^{3+}$ [28]. Another alternative ionic description, which is in good agreement with the saturation magnetic moment, $m_{s}=4 \mu_{B} / f . u$., is $\mathrm{Fe}^{2+}\left(3 \mathrm{~d}^{4}\right)$ and $\mathrm{Mo}^{6+}\left(4 \mathrm{~d}^{0}\right)$ assuming a superexchange between the iron sites. How- 
ever, as stated above, if the valence of Fe is $2+$ then Mo would be in a $4 \mathrm{~d}^{0}$ $6+$ state and hence the compound would be expected to be insulating. This is clearly not observed in the $\mathrm{Sr}_{2} \mathrm{FeMoO}_{6}$ transport data. Mössbauer data has been interpreted in terms of a mixed Fe valence with an average value near 2.5+ [29]. Thermoelectric power measurement on electron doped $\mathrm{Sr}_{1.5} \mathrm{La}_{0.5} \mathrm{FeMoO}_{6}$ [30] showed that this technique could be exploited to probe the doping state of the double perovskite and therefore potentially provide further insight into the electronic states. Clearly some additional work should be performed on $\mathrm{Sr}_{2} \mathrm{FeMoO}_{6}$ and related compounds to further probe the Fe and Mo electronic states.

The consequence of the gap for only one spin is that the charge carriers at $E_{F}$ are fully spin polarised. A $100 \%$ spin polarisation of the charge carriers at room temperature is desirable to enhance the tunneling magnetoresistance of polycrystalline compounds for practical application. Even at low field ( $<1$ Tesla $(\mathrm{T})$ ) and room temperature, a large spin-dependent tunneling magnetoresistance is observed in the double perovskites (see figure 2.2(b)) as the charge carriers tunnel more easily between the grains if the neighbouring grains have the same magnetic orientation. The magnetic domains orientate in the direction parallel to the field and this has the effect of reducing the spin scattering in the tunneling junctions and therefore a drop in the resistivity is observed when a magnetic field is applied. The $\mathrm{Sr}_{2} \mathrm{FeMoO}_{6}$ double perovskite exhibits large magnetoresistance $(\backsim-10 \%)$ at room temperature and large field while at liquid helium temperature it can show a magnetoresistance as large as $\backsim-42 \%$ (see figure 2.2). However, the correlation between the magnetoresistance and the other properties is not clearly established. For example, some investigators have found a maximum low field $\mathrm{MR}$ in $\mathrm{Sr}_{2-x} \mathrm{Ba}_{x} \mathrm{FeMoO}_{6}$ of $\sim-20 \%$ at $0.8 \mathrm{~T}$ and $80 \mathrm{~K}$ for $x=1$ [31]. It is not yet clear if the large MR arises from the Ba substitution or if it is due to reduced disorder near the grain boundaries. 


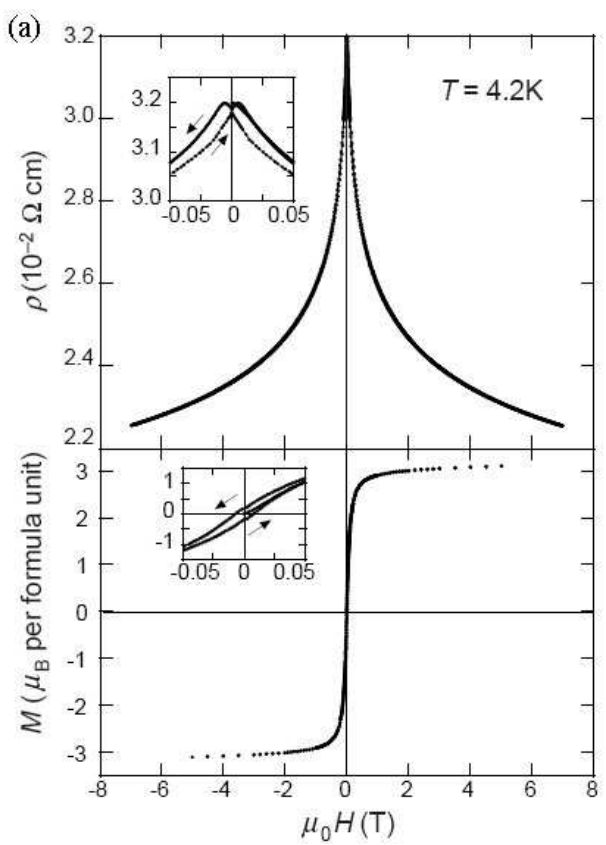

(b)

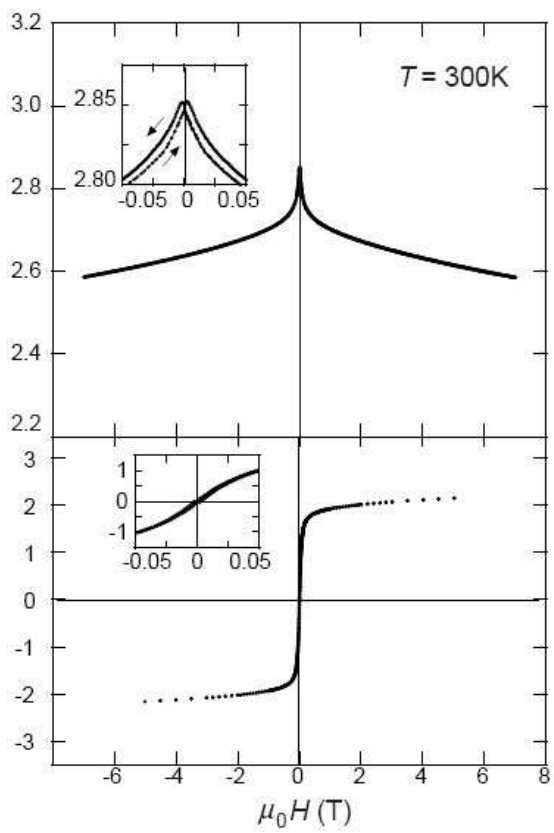

Figure 2.2: Magnetoresistance and magnetisation data at $4.2 \mathrm{~K}$ (a) and 300 $\mathrm{K}$ (b) from Kobayashi et al. in 1998 [11]. The magnetoresistance at $7 \mathrm{~T}$ is $\sim$ $-42 \%$ and $\backsim-10 \%$ at $4.2 \mathrm{~K}$ and $300 \mathrm{~K}$ respectively.

The flexibility of the $\mathrm{BB}^{\prime}$ combination [23] in the $\mathrm{A}_{2} \mathrm{BB}^{\prime} \mathrm{O}_{6}$ double perovskite gives rise to a wide range of possibilities and hence leads to various magnetic and transport properties. There is still an open debate concerning the origin of the exchange coupling between the $\mathrm{BB}^{\prime}$ sites and more data and interpretation are required to fully understand the physics behind these materials and therefore use them for spintronics applications. Due to a strong coupling of the electrons, even a small partial substitution on the A, B and B' sites will have an effect on the orbitals overlap and therefore alter the properties of the materials. Therefore, small chemical changes on the various sites could be used as a tool to probe the transport and magnetic properties in the double perovskite and hence further understand these compounds. 


\subsection{Strontium iron oxide, $\mathrm{SrFeO}_{3-\delta}$}

The transition metal oxides $\mathrm{ABO}_{3}$ contain a alkaline-earth ion, A (e.g. Ba, $\mathrm{Sr}$ ) and a transition metal, B (e.g. Mn, Co, Fe) surrounded by oxygen octahedra. These compounds have been of interest for decades as they exhibit unusual properties. For instance, the $\mathrm{AMnO}_{3}$ compounds have been studied in detail since the discovery of the colossal magnetoresistance. Perovskite manganites exhibit interesting magnetic and transport behaviours resulting from competing charge and orbital ordering. Even a small substitution concentration on one of the sites or a change in the oxygen content could lead to a distinct variation in structural, magnetic and electronic behaviours. For example, $\mathrm{LaMnO}_{3-\delta}$ is an antiferromagnetic insulator, which exhibits a pronounced Jahn-Teller (JT) distortion as the electrons are sufficiently localised [32]. The partial substitution of Fe on the Mn site increases the $e-O: 2 p_{\sigma}-e$ interactions and the localised $e$ orbitals become a narrow $\sigma^{*}$ band [33]. A metallic-type behaviour is observed for complete Fe substitution, namely $\mathrm{SrFeO}_{3}$ [34]. The Fe-based perovskites have recently drawn attention as they could provide further insight into the other perovskite systems. Figure 2.3 shows the crystal structure of the Fe-based perovskite oxide.

The $\mathrm{SrFeO}_{3}$ material has a transition temperature at $T_{N} \backsim 140 \mathrm{~K}[35,36]$ and shows an helical magnetic spin structure with the helicoid vector parallel to the [111] direction and does not exhibit any sign of a JT distortion down to $4.2 \mathrm{~K}$ [34]. On the other hand, oxygen deficient $\mathrm{SrFeO}_{3-\delta}$ is known to display a rich phase diagram for 3- $\delta$ ranging from 3 to 2.5. There is evidence from Mössbauer measurements that an homogenous phase with random oxygen vacancies does not exist $[37,35]$, and that instead there is a mixture of cubic $\mathrm{SrFeO}_{3}$, tetragonal $\mathrm{Sr}_{8} \mathrm{Fe}_{8} \mathrm{O}_{23}(3-\delta=2.875)$ and orthorhombic $\mathrm{Sr}_{4} \mathrm{Fe}_{4} \mathrm{O}_{11}(3-\delta=2.75)$ phases.

The end member, cubic $\mathrm{SrFeO}_{3}$, contains well-ordered $\mathrm{FeO}_{6}$ octahedra and a Fe valence of 4+ (figure 2.4(a)). As stated above, it displays helical 


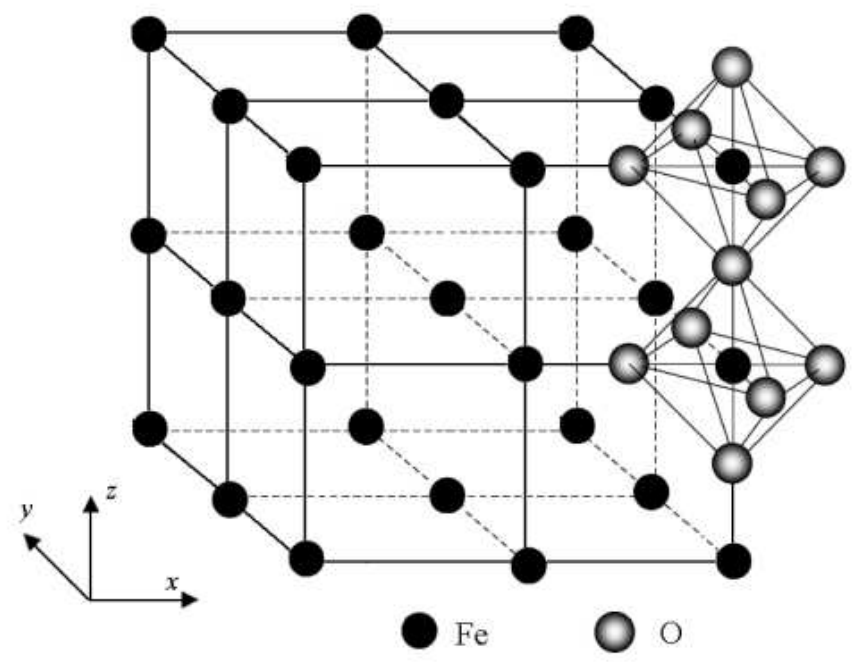

Figure 2.3: Sketch of the $\mathrm{AFeO}_{3}$ perovskite structure. The A element is not shown in the centre of each of the cells for clarity.

antiferromagnetic order at $\sim 140 \mathrm{~K}$ [34] and a high conductivity [38]. The $\mathrm{Sr}_{4} \mathrm{Fe}_{4} \mathrm{O}_{11}$ phase displays antiferromagnetism below $240 \mathrm{~K}$ and a semiconductor-like resistivity [39]. It contains $\mathrm{FeO}_{5}$ units that form dimers and distorted $\mathrm{FeO}_{6}$ octahedra, where the Fe valences are believed to be 3+ and $4+$ respectively (see figure 2.4(c)). The intermediate $\mathrm{Sr}_{8} \mathrm{Fe}_{8} \mathrm{O}_{23}$ phase orders antiferromagnetically near $70 \mathrm{~K}$ and displays an order of magnitude resistivity increase below this temperature [40]. It contains $\mathrm{Fe}(1)$ ions that are five-fold coordinated by oxygen and form dimers [41]. They are adjacent to $\mathrm{Fe}(2)$ sites with distorted $\mathrm{FeO}_{6}$ octahedra that are adjacent to $\mathrm{Fe}(3)$ sites with $\mathrm{FeO}_{6}$ octahedra (figure 2.4(b)). It has been argued that the $\mathrm{Fe}(1)$ and $\mathrm{Fe}(3)$ ions have a 4+ valence and the $\mathrm{Fe}(2)$ sites have a 3.5+ valence and that the increased resistivity near $70 \mathrm{~K}$ arises from ChargeOrdering (CO) of the $\mathrm{Fe}(2)$ ions to $\mathrm{Fe}^{3+}$ and $\mathrm{Fe}^{4+}[40,37]$.

Other compounds in this family such as $\mathrm{CaFeO}_{3}$ exhibit Charge Disproportion (CD) between $\mathrm{Fe}^{3+}$ and $\mathrm{Fe}^{5+}$ at low temperature. This $\mathrm{CD}$ has 
also been observed in other materials such as $\mathrm{La}_{1 / 3} \mathrm{Sr}_{2 / 3} \mathrm{FeO}_{3}$ following a sequence of $\mathrm{Fe}^{3+}(\uparrow), \mathrm{Fe}^{3+}(\uparrow), \mathrm{Fe}^{5+}(\uparrow), \mathrm{Fe}^{3+}(\downarrow), \mathrm{Fe}^{3+}(\downarrow), \mathrm{Fe}^{5+}(\downarrow)$ along the (111) direction. $\mathrm{La}_{1 / 3} \mathrm{Sr}_{2 / 3} \mathrm{FeO}_{3}$ undergoes a charge-ordering phase transition at $198 \mathrm{~K}$. Substitutions on the A site with smaller ionic radii ions (e.g. Pr, Nd) causes the $p-d$ hybridisation interaction to decrease and $T_{C O}$ drops [42]. The CO transition completely vanishes with Sm and Gd substitution due to the greater lattice distortion and Variable Range Hopping (VRH) is observed [42].

Initially, the $\mathrm{SrFeO}_{3-\delta}$ compound was of interest for our research as it is one of the double perovskite minority phases (see chapter 3) but it turned out to be a case study on its own. For instance, the relation between charge-ordering and the magnetic behaviour is still not clearly understood in $\mathrm{SrFeO}_{3-\delta}$. The magneto-transport data exhibits unexpected behaviour such as negative and positive magnetoresistance around the charge-ordering temperature with increasing oxygen deficiency in

$\mathrm{SrFeO}_{3-\delta}$ [37]. As mentioned in a previous report [40], this $\mathrm{CO}$ is reminiscent of the Verwey transition observed in $\mathrm{Fe}_{3} \mathrm{O}_{4}$ but additional experiments are necessary to ascertain if a similar interpretation is valid in $\mathrm{SrFeO}_{3-\delta}$. Thermoelectric power measurements have never been published for this compound but could clearly provide further information about the charge-ordering state as seen in the $\mathrm{Fe}_{3} \mathrm{O}_{4}$ system $[43,44]$. In addition, there are still some unsolved questions concerning the magnetic properties of $\mathrm{SrFeO}_{3-\delta}$. Further magnetisation data should be taken over a broader range of oxygen content, ideally down to $\mathrm{SrFeO}_{2.5}$, in order to understand the various magnetic contributions from the different iron sites. 
(a) $\mathrm{SrFeO}_{3}$

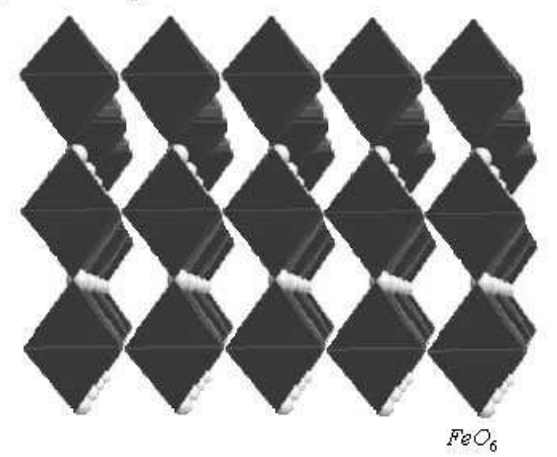

(b) $\mathrm{Sr}_{8} \mathrm{Fe}_{8} \mathrm{O}_{23}$

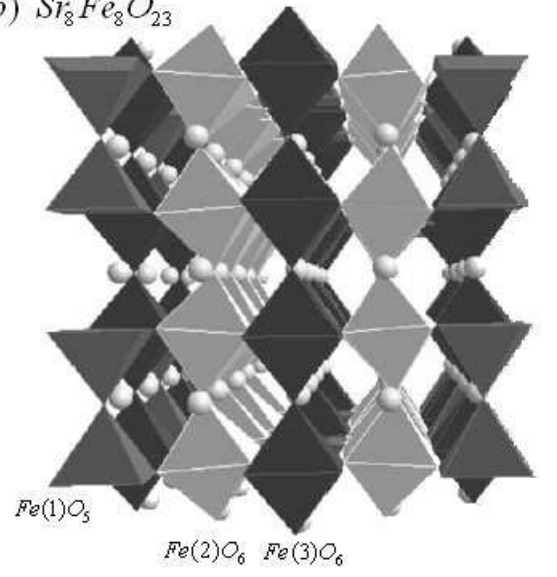

(c) $\mathrm{Sr}_{4} \mathrm{Fe}_{4} \mathrm{O}_{11}$

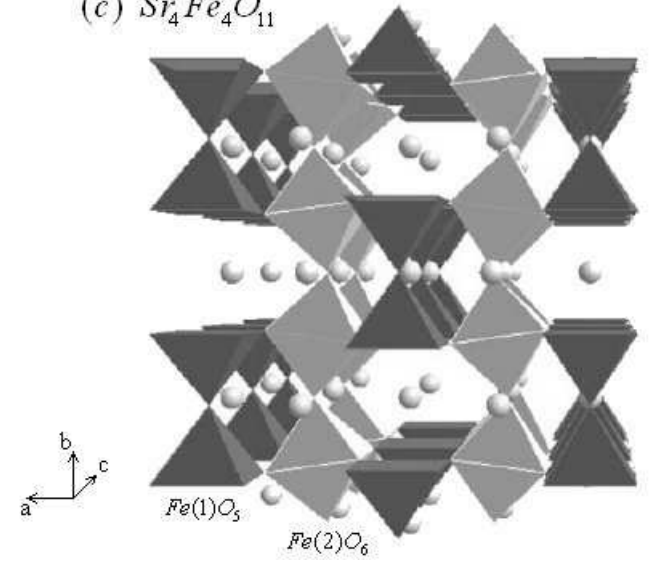

Figure 2.4: (a) $\mathrm{SrFeO}_{3}$, (b) $\mathrm{Sr}_{4} \mathrm{Fe}_{4} \mathrm{O}_{11}$ and (c) $\mathrm{Sr}_{8} \mathrm{Fe}_{8} \mathrm{O}_{23}$ crystal structures. $\mathrm{SrFeO}_{3}$ is made of ideal $\mathrm{FeO}_{6}$ octahedra shown in black. $\mathrm{Sr}_{4} \mathrm{Fe}_{4} \mathrm{O}_{11}$ are made of $\mathrm{Fe}(1) \mathrm{O}_{5}$ dimers (grey), distorted $\mathrm{Fe}(2) \mathrm{O}_{6}$ (light grey) and $\mathrm{Fe}(3) \mathrm{O}_{6}$ octahedra (black). $\mathrm{Sr}_{8} \mathrm{Fe}_{8} \mathrm{O}_{23}$ is composed of $\mathrm{Fe}(1) \mathrm{O}_{5}$ (grey) and distorted $\mathrm{Fe}(2) \mathrm{O}_{6}$ (light grey) [37]. 


\section{Chapter 3}

\section{Experimental details}

This chapter outlines the double perovskite $\mathrm{A}_{2} \mathrm{BMoO}_{6}$ and strontium iron oxide $\mathrm{SrFeO}_{3-\delta}$ sample preparation details as well as the various instruments used for their structural, transport and magnetic characterisations.

\subsection{Sample preparation}

\subsubsection{Double perovskites}

Double perovskites, $\mathrm{A}_{2} \mathrm{BMoO}_{6}$, were prepared by a solid-state reaction method from stoichiometric mixes of $\mathrm{A}\left(\mathrm{NO}_{3}\right)_{2}(\mathrm{~A}=\mathrm{Sr}$, $\mathrm{Ba}$ and $\mathrm{La}), \mathrm{B}_{2} \mathrm{O}_{3}(\mathrm{~B}$ $=\mathrm{Fe}$ and $\mathrm{Al}$ ) and $\mathrm{MoO}_{3}$. The powders were mixed, ground and de-nitrated at $700{ }^{\circ} \mathrm{C}$ in air for 1 hour. If one of the powders contained water of hydration (e.g. $\mathrm{La}\left(\mathrm{NO}_{3}\right)_{2} 6 \mathrm{H}_{2} \mathrm{O}$ ) then a slower de-nitration ramp was used from 200 to $700{ }^{\circ} \mathrm{C}$ in order to remove all the water of hydration from the powder. The powder was then pressed, at $40000 \mathrm{kPa}$, into pellets using a die. The typical dimensions of the pellet were $10 \mathrm{~mm}$ in diameter and 2 mm thick.

The pellets were placed in a crucible in air at $1200{ }^{\circ} \mathrm{C}$ for 4 hours. In order to avoid any cracks due to thermal shocks the pellets were removed from the furnace at low temperature $\left(\backsim 100{ }^{\circ} \mathrm{C}\right)$. After this step the two 
preliminary phases were present, namely $\mathrm{AMoO}_{4}$ and $\mathrm{AFeO}_{3-\delta}$ and are shown by $(+)$ and $(x)$ respectively in figure 3.1 for a strontium-based compound.

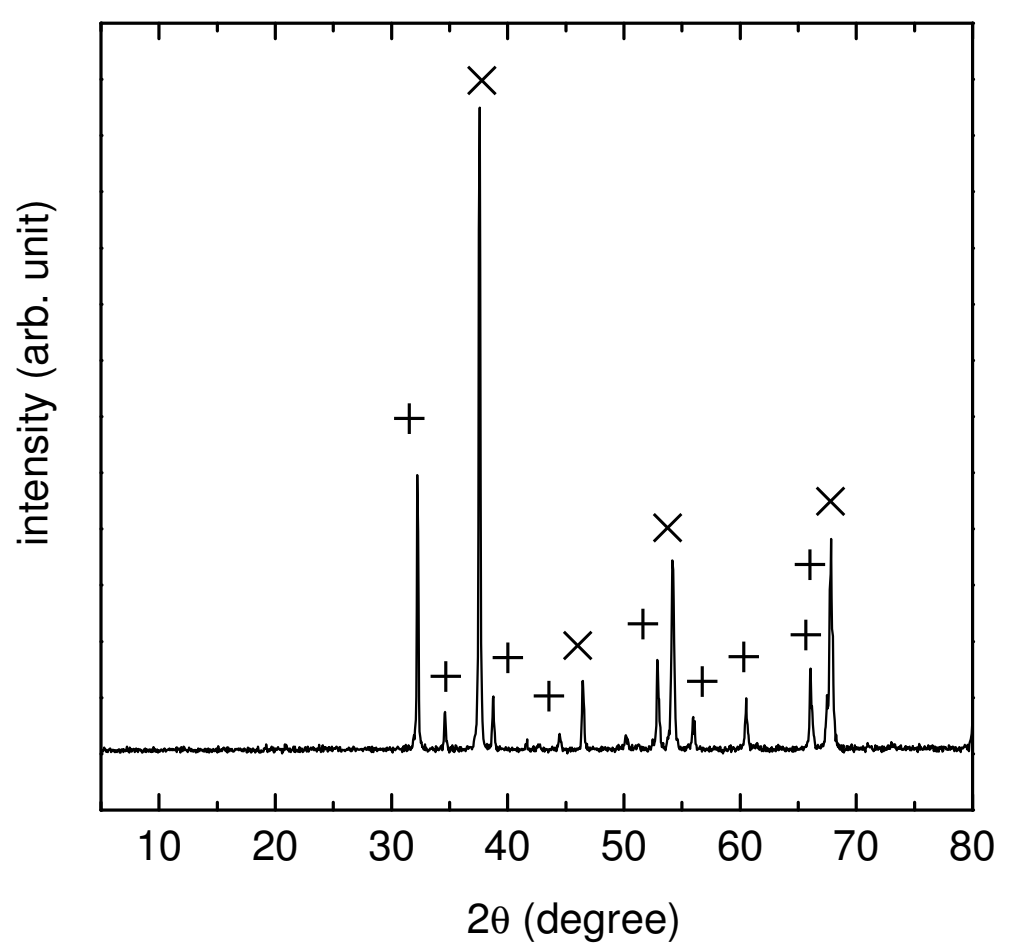

Figure 3.1: X-ray diffraction pattern using $C o K_{\alpha}$ radiation after the intermediate step in air. It shows the two preliminary phases of $\mathrm{SrMoO}_{4}(+)$ and $\mathrm{SrFeO}_{3-\delta}(\mathrm{x})$.

The samples were then ground, pressed into pellets and then sintered at a temperature of between 1000 to $1200{ }^{\circ} \mathrm{C}$ in an atmosphere of $5 \% \mathrm{H}_{2}$ $95 \% \mathrm{~N}_{2}\left(\mathrm{H}_{2} / \mathrm{N}_{2}\right)$ for $3>t>6$ hours. The flow of $\mathrm{H}_{2} / \mathrm{N}_{2}$ was turned on 30 minutes before going up in temperature to ensure that the tube contained only $\mathrm{H}_{2} / \mathrm{N}_{2}$ gas and not air. Even though a range of temperatures seems to work for the sintering of the double perovskite, results from various experiments (not presented here) showed that $1100{ }^{\circ} \mathrm{C}$ is the more appro- 
priate temperature to obtain single phase material. The latter process was repeated one more time, after regrinding and re-pelleting, in order to remove any minority phase XRD peaks and obtain homogeneous samples. Depending on the composition of the sample, checked by $x$-ray diffraction, the $\mathrm{H}_{2} / \mathrm{N}_{2}$ sintering was repeated several times. Note that the double perovskite can be formed by directly using the $\mathrm{H}_{2} / \mathrm{N}_{2}$ treatment. However it was found that pre-firing in air is necessary to obtain denser samples that are more stable in ambient atmosphere over time [45].

\subsection{2 $\mathrm{SrFeO}_{3-\delta}$}

Polycrystalline samples of $\mathrm{SrFeO}_{3-\delta}$ were synthesised by solid-state reaction from stoichiometric mixtures of $\mathrm{Sr}\left(\mathrm{NO}_{3}\right)_{2}$ and $\mathrm{Fe}_{2} \mathrm{O}_{3}$. The powder was de-nitrated at $700{ }^{\circ} \mathrm{C}$, pressed into pellets at around $40000 \mathrm{kPa}$ and then placed in air at $1200{ }^{\circ} \mathrm{C}$ for 24 hours. Slow cooling was performed before removing the samples out of the furnace. The pellets were then ground for better homogeneity and then re-sintered in air for 12 hours at a similar temperature.

In order to load the samples with oxygen, the pellets were placed at high pressure, $6000 \mathrm{kPa}$, in pure oxygen for 24 hours. To diffuse the oxygen throughout the samples the high pressure treatment needed to be performed at high temperature $\left(700{ }^{\circ} \mathrm{C}\right)$. The samples were then slow cooled to room temperature. To vary the oxygen content $(\delta)$, the samples were then placed in $7.5,2$ or $0.2 \%$ oxygen in $\mathrm{N}_{2}$. As well as the atmosphere composition, the temperature could also be changed, typically from 400 to $600{ }^{\circ} \mathrm{C}$, to obtain different $\delta$ values. The higher $\mathrm{N}_{2}$ content and temperature, the more oxygen that could be removed from the samples. Therefore in order to obtain various oxygen deficient samples we varied these two parameters and obtained samples from $\mathrm{SrFeO}_{2.95}$ to $\mathrm{SrFeO}_{2.72}$.

Determination of the oxygen content is usually performed by Thermal Gravimetry Analysis (TGA) but this instrument was not available. There- 
fore in order to determine the exact change in the oxygen content $(\Delta \delta)$, the mass of the samples were measured before $\left(m_{i}\right)$ and after the $\mathrm{N}_{2}$ treatment $\left(m_{f}\right)$. From these changes the $\Delta \delta$ can be extracted using the following equation,

$$
\begin{gathered}
m_{f} m_{\mathrm{SrFeO}_{3-\Delta \delta}}=m_{i} m_{\mathrm{SrFeO}_{3}} \\
\Delta \delta=\frac{\left(\frac{m_{i}}{m_{f}}\right) m_{\mathrm{SrFeO}_{3}}-m_{\mathrm{Sr}}-m_{\mathrm{Fe}}-3 m_{O}}{m_{O}}
\end{gathered}
$$

where $m_{i}$ and $m_{f}$ are the initial and final masses respectively, $m_{S r}, m_{F e}$, $m_{\mathrm{O}}, m_{\mathrm{SrFeO}_{3}}$ are the atomic masses of $\mathrm{Sr}, \mathrm{Fe}, \mathrm{O}$ and $\mathrm{SrFeO}_{3}$ respectively. Note that this provides a change in the oxygen content, $\Delta \delta$, and not the actual oxygen content, $\delta$, which would be required to compare our results with the data in the literature. We show in chapter 7 that the magnetic susceptibility provides a good estimate of the $\delta$ value in the as-prepared materials.

\subsection{X-Ray Diffraction (XRD)}

The phase composition and purity of each sample was determined from $x$-ray diffraction. Monochromatic x-rays are used to determine the interplanar spacing $(d)$ of unknown specimens. The interaction of the x-ray with a sample creates secondary diffracted beams which are related to $d$ following Bragg's law,

$$
n \lambda=2 d \sin \Theta
$$

where $n$ is an integer number, $\lambda$ is the x-ray wavelength, $d$ the interplanar spacing and $\Theta$ is the diffraction angle. The XRD instrument used was a PW 1729 X-ray generator located at Industrial Research Ltd, Wellington, New Zealand with a cobalt tube $\left(C o K_{\alpha}\right.$ radiation) having a wavelength of 1.789 $\AA$. The operating voltage was $40 \mathrm{kV}$ and the current was $20 \mathrm{~mA}$. All the $\mathrm{x}$-ray diffraction patterns were obtained at room temperature. The specimen were abraded uniformly using fine sandpaper and then mounted 
on an aluminium holder, which did not contribute to the background signal. In our polycrystalline samples there is no preferential orientation and therefore all the XRD peaks should be present.

Several parameters such as the angles scanned, integration time and stepping angle were available in the software controlling the instrument. For the characterisation of our samples, the measurement was typically performed from 5 to 80 degrees in 30 minutes, which gives enough accuracy to check the phase(s) in the sample. For the determination of the amount of antisite disorder in the double perovskite (see chapter 4), a scan of 4 hours from 18 to 41 degrees was used and integrated over a longer period of time in order to have an adequate signal to noise ratio.

After the measurement, the Eva software (SOCABIM) and the International Centre for Diffraction Data (ICDD) database was used for the identification of the crystal structure. Two corrections to the obtained patterns were applied: the background subtraction and the $K_{\alpha}$ stripping. Background subtraction was performed to remove the contribution to the pattern from all scattering sources other than coherent diffraction from the crystal lattice. Secondly, $K_{\alpha}$ stripping was necessary as the output of an xray tube is not purely monochromatic and our diffracted beam monochromator has insufficient resolution, each peak in the diffraction pattern is actually an envelope of a closely-spaced doublet - the higher and lower wavelength alpha 1 and 2 lines respectively.

\subsection{Scanning Electron Microscope (SEM)}

The scanning electron microscope was a Jeol JSM-6500F located at Victoria University of Wellington, Wellington, New Zealand. The SEM technique consists of an energetically focused beam of electrons. This highly focused beam is obtained using a series of magnetic lenses. As SEM uses electrons instead of light as used in a standard microscope, the sample has to conduct electricity relatively well. The beam hits the sample and this creates 
the release of secondary electrons that are then counted by a detector and amplified to obtain a reasonable signal. A set of scanning coils moves the beam across the specimen in order to obtain an image of the area under study. The image can be gathered at various magnification generally from 1 to 20000 times. The figure below is an SEM image from a double perovskite sample at a magnification of 10000 .

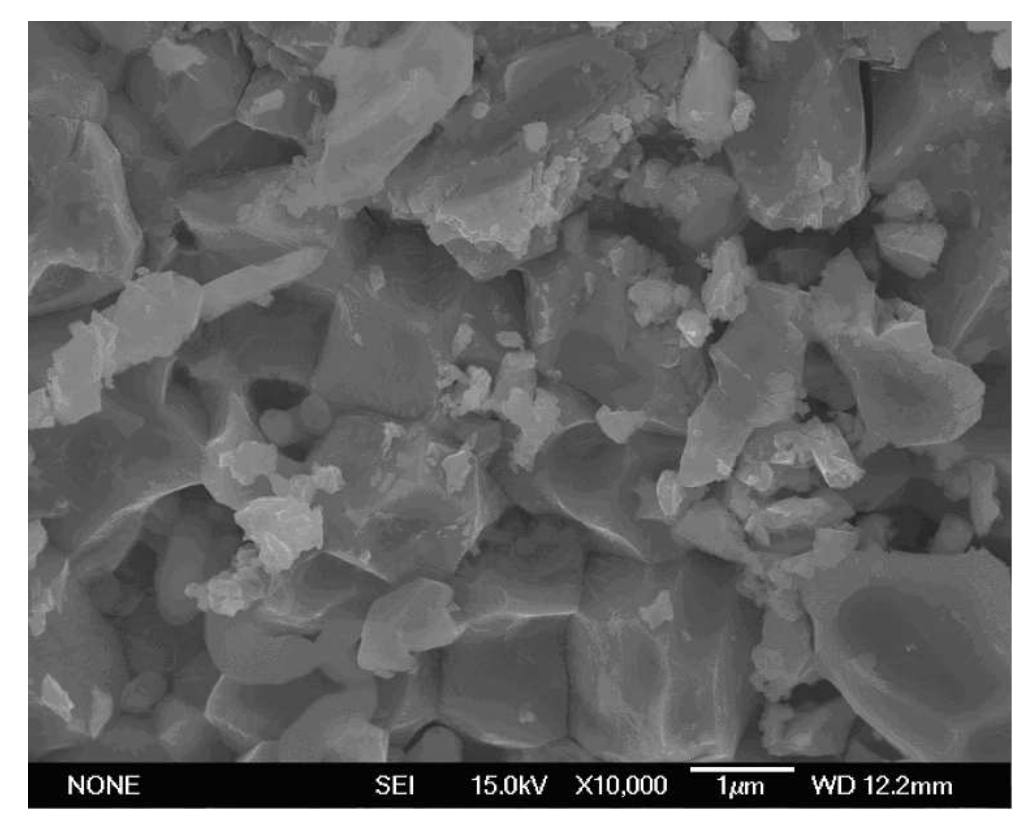

Figure 3.2: Scanning electron microscope image of a $\mathrm{Ba}_{2} \mathrm{FeMoO}_{6}$ sample taken at a 10000 times magnification.

An Energy-Dispersive X-ray Spectrometer (EDS) EX-64175 JMU was attached to the SEM and enables the concentration of each atomic species present in the sample to be estimated. The focused beam of electrons excited the atoms, which release characteristic x-rays. The total energy intensity gives the relative concentration of each element in the zone under study. The software, JED-2200 Analysis Station program, allowed further analysis after the measurement. 


\subsection{Thermoelectric Power (TEP)}

The thermoelectric power is measured by applying a temperature gradient $(\Delta T)$ between two points in a sample, which results in a thermoelectric potential difference $(\Delta V)$ between the high and low temperature region. It should be noted that unlike the resistivity, the thermoelectric power of the polycrystalline ceramics is not affected by high inter-grain boundary resistances. It is affected by inter-grain boundaries only if they have a large thermal resistance because the thermoelectric response is driven by the temperature gradient rather than by the electric field.

Before each measurement the samples were abraded with fine sandpaper in order to have a smooth and flat surface. The sample was placed between two copper plates and pressed to get a good contact resistance. The measurements on each sample were performed several times to obtain the experimental uncertainty. It is also important to note that the samples were removed from the rig and re-abraded between each measurement to insure that there were no problems with interface thermal resistances when compared with the bulk thermal resistance.

The thermoelectric voltage between the $\mathrm{Cu}$ plates was amplified using a commercial DC low noise nanovolt amplifier (EM-electronics A10) and measured by a HP 3478A multimeter. The calibration of the thermoelectric power of the $\mathrm{Cu}$ leads and plates was accomplished using lead $(\mathrm{Pb})$ from ambient to $90 \mathrm{~K}$ and a $\mathrm{YBa}_{2} \mathrm{Cu}_{3} \mathrm{O}_{7-\delta}$ sample (YBCO) below that temperature. This was necessary as the impurities in $S_{P b}$ start to play a dominant role below $90 \mathrm{~K}$ [46]. Note that the calibration of the system was done before I started to use the instrument and was directly incorporated in the program written in Pascal.

A heater was placed on one end of the rig in order to produce the temperature gradient across the sample $(\Delta T)$. Two chromel-gold-iron $(0.07 \%$ $\mathrm{Au})$ differential thermocouple junctions were used to measure $\Delta T$. The thermocouple needs to have a good thermal contact with the $\mathrm{Cu}$ blocks 


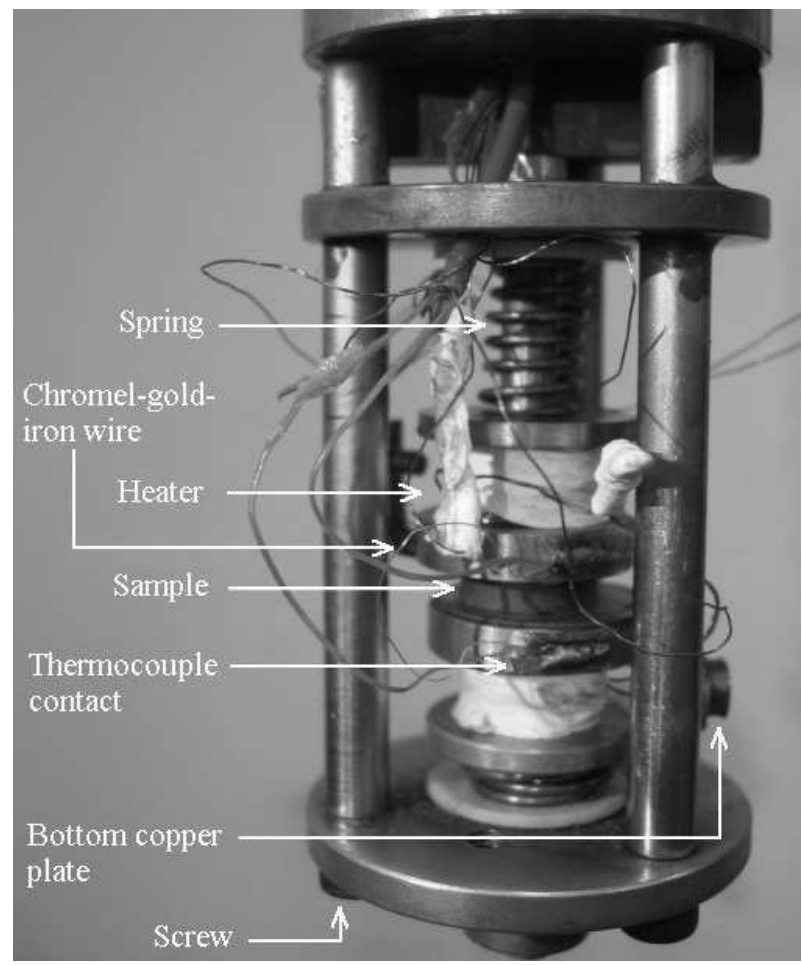

Figure 3.3: Thermoelectric power apparatus showing a sample pressed between the two copper plates. Chromel-gold-iron was used as the thermocouple with a heater placed on the top to apply the temperature gradient. The sample was pressed by using two springs and three screws at the bottom of the rig.

and to be placed as close as possible to the $\mathrm{Cu}$ plates. The experimental assumption is that the thermal resistance of the sample under study is much larger than the equivalent thermal resistance between the thermocouple contact and the $\mathrm{Cu}$ plates. Figure 3.3 shows the thermoelectric power rig with the various components.

The thermoelectric power measurements can be carried out from liquid helium to room temperature using a motor that raised the rig inside a dewar. The temperature was monitored by a silicon diode (CY7-SD7 tem- 
perature sensor, OMEGA). A stabilisation time of around 8 to 10 minutes is required between each motor step, i.e. at each temperature, to ensure that the temperature was stable.

At each temperature the following sequence was performed to measure the thermoelectric power. The program recorded the temperature difference at the two ends and the voltage across the sample. This was typically repeated ten times to obtain a set of $\mathrm{V}$ versus $\Delta T$ points. Then a current passed through the coil to heat up one end of the sample and after a minute another set of $\mathrm{V}$ versus $\Delta T$ points were taken. A linear regression was performed between these two sets of points in order to derive the gradient which is the thermoelectric power $(S)$ usually expressed in $\mu \mathrm{V} / \mathrm{K}$.

\subsection{Temperature dependence of the resistivity $(\rho)$}

The resistance $(R)$ in ohms $(\Omega)$ versus temperature was measured using the four terminal method. This method consists of two leads, on the outside, used to supply the current to the unknown specimen and two others, in the inside, to measure the voltage drop across the sample. The current was supplied by a Keithley 224 programmable current source while the measured voltage was recorded with a HP 3478A multimeter. The current flows through the outside leads and the sample but negligible current flows in the voltmeter's leads due its large internal resistance. Therefore, this technique eliminates contact-resistance errors and it is necessary when the resistance is small $(R<10-100 \Omega)$.

The electrical contacts were clamped onto the sample or silver paint was used. The measurements were performed from low temperature (usually at around $20 \mathrm{~K}$ ) to room temperature by lifting the probe from the bottom of a dewar using a programmable stepper motor. The temperature was monitored by a silicon diode (CY7-SD7 temperature sensor, OMEGA) placed as close as possible to the sample $(\backsim 2 \mathrm{~mm})$ and having a good ther- 
mal contact. A HP 3478A multimeter was used to record the voltage of the diode and then converted to a temperature value using the calibration table provided by OMEGA.

A set of three currents was used in order to check the ohmic behaviour of the measurements. In addition, a positive and negative value for each current was applied. This reverse current technique is used to remove any thermoelectric voltage or voltage offsets; this technique is especially advisable when measuring low voltages. The two output voltages, from the positive and negative currents, were then subtracted from each other and divided by two. This latter value was then divided by the current to obtain the resistance. The data was recorded in an American Standard Code for Information Interchange (ASCII) file for later analysis.

This measurement could be performed on an arbitrary shaped pellet for the resistance or on a well defined bar-shape having straight contact pads to obtain the resistivity. In the case of the bar-shape sample, the resistance values were converted to resistivity using the following equation,

$$
\rho=\frac{R A}{L}
$$

where $\rho$ is the resistivity in $\Omega . \mathrm{cm}, R$ is the resistance in $\Omega, A$ is the cross section area in $\mathrm{cm}^{2}$ of the bar-shape and $L$ is the distance between the two voltage contacts in $\mathrm{cm}$.

\subsection{Magnetoresistance (MR)}

\subsubsection{Low field magnetoresistance}

The magnetoresistance is the change of the resistivity on application of an external magnetic field, $B$. We define the magnetoresistance by,

$$
M R=\frac{\rho(H)-\rho(0)}{\rho(0)}
$$


where $\rho(H)$ and $\rho(0)$ are the resistivity under an applied magnetic field and at zero field respectively. Note that you can use the resistance instead of the resistivity. The MR data in this thesis are expressed in percentage. Magnetoresistance can occur from many sources (e.g. orbital, anisotropy, spin-flip scattering) but in our case we are interested in the Tunneling Magnetoresistance (TMR). In the TMR situation it is understood that if all the magnetic domains are aligned along the external magnetic field, it is easier for the electrons to tunnel through the insulating barrier as the neighbouring domains will have available spin states as long as the material is metallic. In that case the resistance is low. However, if the magnetic domains are randomly orientated then the electrons will not have an equivalent spin state in the neighbouring domains and this will result in a high resistance. The MR is an important parameter in the double perovskite and therefore the development of a magnetoresistance apparatus was necessary for the characterisation of our samples.

An instrumental set-up was developed to measure the magnetoresistance of ceramic samples. The hardware, interface and software were put together in order to perform MR measurements at room $(\backsim 300 \mathrm{~K})$ and liquid nitrogen $(\backsim 77 \mathrm{~K}$ ) temperature. A four-probe clamp was placed in a uniform longitudinal magnetic field, $B$, produced by a magnet having a maximum field of $1 \mathrm{~T}$ with a step size of $10^{-4} \mathrm{~T}$. Figure 3.4 shows the experimental set-up at room temperature with a ceramic pellet mounted on the probe placed in the centre of the magnetic field. For the measurement at $77 \mathrm{~K}$, the probe was dipped into liquid nitrogen using a small glass dewar placed between the poles.

A current was passed through the sample and the voltage was measured. This was done under various applied magnetic fields to obtain the magnetoresistance. The sequence of measurements as well as the electronic hardware were similar to those used in the resistivity measurements. 


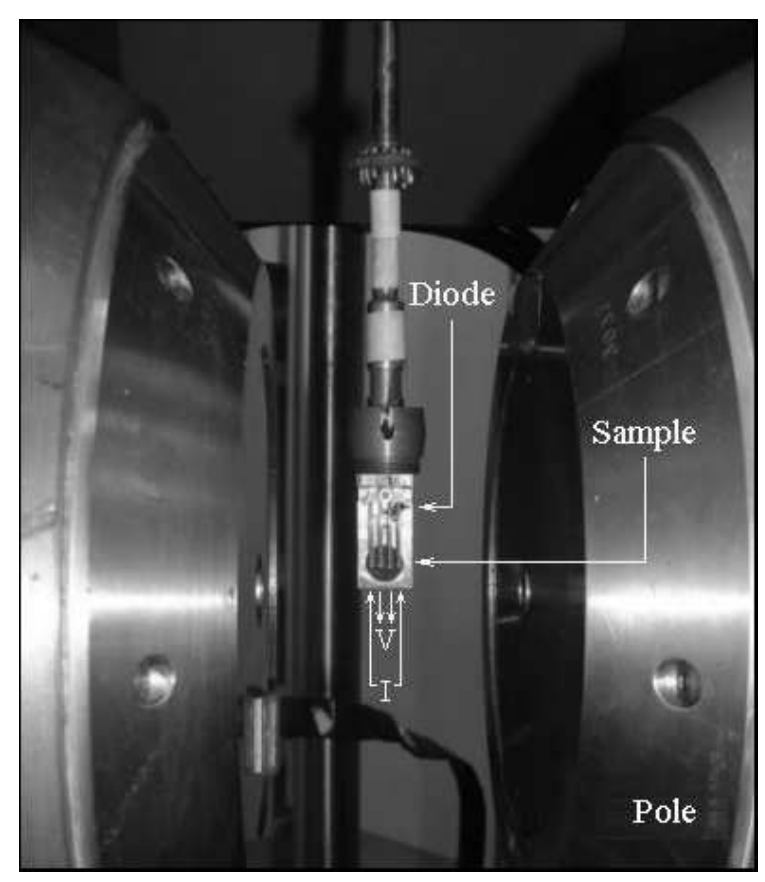

Figure 3.4: Magnetoresistance apparatus at room temperature.

The electronic instruments were connected via a General Purpose Interface Bus (GPIB) interface to a computer and a program in LabView (National Instrument) was written in order to control and record the data. The external field produced by the magnet was changed manually as no interface was available to control it. The measurement at each magnetic field was repeated a number of times to get an average and the experimental uncertainty. The user interface displayed the results and these were recorded at the same time in an ASCII file for later analysis. 


\subsection{Superconducting QUantum Interference Device Magnetometer}

(SQUID)

\subsubsection{High field magnetoresistance up to $12 \mathrm{~T}$}

Magnetoresistance measurements up to $12 \mathrm{~T}$ and down to liquid helium were performed at the École Polytechnique Fédérale de Lausanne (EPLF) in Switzerland. The longitudinal applied magnetic field from a superconducting magnet (Cryogenics) was variable from 0 to $12 \mathrm{~T}$ with $10^{-1} \mathrm{~T}$ steps. The sample chamber was immersed in helium liquid and controlled by a heater in order to vary the temperature from 4.2 to $300 \mathrm{~K}$. The temperature controller was a Lakeshore 340. A pump was used to avoid any condensation in the dewar and to cool down below helium temperature if necessary. The sample holder could fit up to six samples and was connected using the usual four terminal method. The current source was supplied by a Keithley 224 and the voltage was recorded by a Keithley 182 nanovoltmeter. A program in Visual Basic was used to control and record the instruments. An ASCII file was obtained at the end of the measurement for later analysis.

\subsection{Superconducting QUantum Interference De- vice Magnetometer (SQUID)}

A Superconducting QUantum Interference Device (SQUID) MPMS from Quantum Design is the most sensitive instrument to measure magnetic properties and can measure magnetic moments down to $10^{-8} \mathrm{emu}$. The SQUID was located at Industrial Research Ltd and was used to perform all the magnetic measurements presented in this thesis such as the temperature dependence and field sweeps. The measurement can be carried out at temperatures from 800 to $5 \mathrm{~K}$ and in magnetic fields of up to $7 \mathrm{~T}$.

As the sample moves through a set of detecting coils (see figure 3.5(a)), the magnetic moment from the sample induces an electric current in these coils. There are four detecting coils: the top one wound clockwise, two in the centre anti-clockwise and the bottom one clockwise. This configura- 


\subsection{Superconducting QUantum Interference Device Magnetometer} (SQUID)

(a)

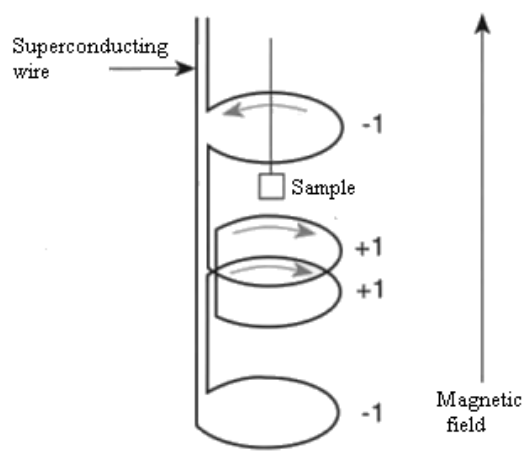

(b)

SQUID response

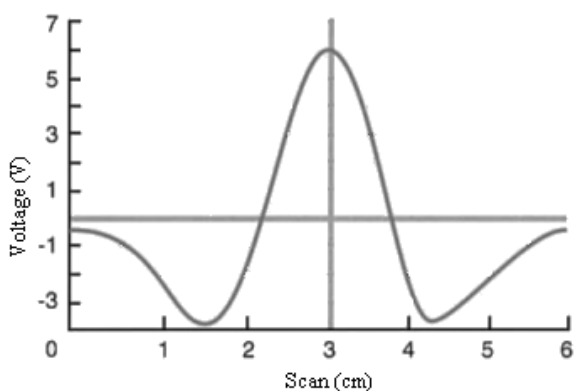

Figure 3.5: (a) Configuration of the second-order gradiometer superconducting detection coil. (b) The output of the SQUID as a magnetic dipole is moved through the second-order gradiometer pickup coil [47].

tion, known as a second-order gradiometer, is used to reduce any possible noise and offsets in the detection circuit produced by the external superconducting magnet. The current picked by the detecting coil is then converted to a voltage via the rf SQUID detector. This voltage is measured at various positions along the specified scan length. This is performed many times to increase the signal-to-noise ratio. This response is known as the dipole response (figure 3.5(b)) and a fit of this curve is done in order to extract the magnetic moment, if the system has been previously calibrated. Calibration using a palladium sample of a known mass and susceptibility was performed regularly. ac magnetisation measurements can also be performed using the MPMS SQUID magnetometer.

The SQUID gives the total magnetic moment in emu, $m^{e m u}$, and we measured the mass (mass) of the sample to obtain the magnetic moment per formula unit, $m$,

$$
m=\frac{m^{e m u} M_{r}}{\text { mass }}
$$

where $M_{r}$ is the relative atomic weight in grams of one formula unit. The magnetic data are also presented as magnetisation $(M)$ and susceptibility 


\subsection{Superconducting QUantum Interference Device Magnetometer} (SQUID)

$(\chi)$, and are obtained by using the following equations,

$$
\begin{gathered}
M=\frac{m^{e m u} \rho}{m a s s} \\
\chi=\frac{M}{H}
\end{gathered}
$$

where $\rho$ is the density and $H$ the applied magnetic field. Note that all of these parameters are expressed in System International (SI) units throughout the thesis.

An oven insert can be placed instead of the standard environment in order to performed magnetisation measurement up to $800 \mathrm{~K}$. A home made sample holder was prepared using copper wires $(0.6 \mathrm{~mm}$ diameter) to carry out these measurements as the standard holder is a plastic straw and would melt above $400 \mathrm{~K}$. This holder was made of four wires twisted together using a hand drill. By using this technique we were able to obtain a nearly perfect thread of wires having a uniform cross section to avoid any background signal. A cage was formed in the middle of the thread to hold the sample in place. Some parallel work was carried out (not presented in this thesis) using this home made probe on the ruthenate cuprate $\mathrm{Ru}_{1-y} \mathrm{M}_{y} \mathrm{Sr}_{2} \mathrm{Eu}_{2-x} \mathrm{Ce}_{x} \mathrm{Cu}_{2} \mathrm{O}_{10+\delta}(\mathrm{M}=\mathrm{Nb}, \mathrm{Sn})$ compounds and the results and analysis were published in Physical Review B [48]. 


\section{Chapter 4}

\section{Isoelectronic substitution in $\mathbf{S r}_{2-x} \mathbf{B a}_{x} \mathbf{F e M o O}_{6}$}

The half-metallic double perovskite $\mathrm{Sr}_{2} \mathrm{FeMoO}_{6}$ has recently attracted interest due to the large Magnetoresistance (MR) at room temperature [11]. For application purposes it is desirable to maximize the Low Field Magnetoresistance (LFMR). The large negative MR in polycrystalline compounds is believed to originate from the tunneling of spin-polarised electrons across insulating grain boundaries, so that the intra-grain magnetisation and the inter-grain tunneling characteristics are clearly of importance in the process. However it has recently been suggested that the intra-grain magnetisation is different from the bulk near the grain boundaries, and that this influences the magnetoresistance [49].

The variation of the $\mathrm{A}$ (alkaline-earth ion) in $\mathrm{A}_{2} \mathrm{FeMoO}_{6}$ has a direct influence on the magnetic properties of the double perovskite [50,21]. This substitution affects the orbital overlap and the exchange coupling between the Fe and Mo. A direct consequence of this is a change in the Curie temperature $\left(T_{c}\right)$ from $400-420 \mathrm{~K}$, for $\mathrm{Sr}^{2+}$, to $310-367 \mathrm{~K}_{\text {for }} \mathrm{Ba}^{2+}$ [31, 23]. Habib et al. have recently reported a systematic decrease in $T_{c}$ and an increase in the saturation magnetic moment $\left(m_{s}\right)$ of $\mathrm{Sr}_{2-x} \mathrm{Ba}_{x} \mathrm{FeMoO}_{6}$ as the barium concentration increases, but they interestingly found a maximum LFMR 
of $\sim-20 \%$ at $0.8 \mathrm{~T}$ and $80 \mathrm{~K}$ for $x=1$ [31]. It is not yet clear if the large $\mathrm{MR}$ arises from the A site substitution or if it is due to reduced disorder near the grain boundaries. Furthermore, previous work has shown that samples with enhanced MR also have higher resistivities, in agreement with the grain-boundary-tunneling scenario. Thus, in order to clarify the correlation between the LFMR, magnetic moment and resistivity we synthesised $\mathrm{Sr}_{2-x} \mathrm{Ba}_{x} \mathrm{FeMoO}_{6}$ compounds and report the results from structural, transport and magnetic measurements in this chapter. Two papers reporting this work have been published in Physica B [51] and Journal of Magnetism and Magnetic Materials [52].

\subsection{Structural characterisation}

\subsubsection{X-ray diffraction and degree of antisite disorder}

The crystallographic characterisation of the samples was performed by x-ray diffraction measurements using $\mathrm{CoK}_{\alpha}$ radiation at room temperature. Figure 4.1 shows the XRD patterns for the $\mathrm{Sr}_{2-x} \mathrm{Ba}_{x} \mathrm{FeMoO}_{6}(x=0,1$ and 2) samples. The two references patterns for $\mathrm{Sr}_{2} \mathrm{FeMoO}_{6}(\mathrm{SFMO})$ and $\mathrm{Ba}_{2} \mathrm{FeMoO}_{6}$ (BFMO) are also shown in black and grey respectively in figure 4.1. We measured two $\mathrm{Ba}_{2} \mathrm{FeMoO}_{6}$ samples to see if we could obtain different transport and magnetic properties for samples having the same alkaline-earth ion. The XRD data match the reference patterns for single phase double perovskite (see bottom panel of figure 4.1) and a consistent change from tetragonal (I4/mmm space group) with $a=5.59 \AA$ and $c=7.90$ $\AA$ to cubic (Fm3m space group) with $a=8.06 \AA$ symmetry is observed with the increase of the ionic radii from $\mathrm{Sr}^{2+}=1.26 \AA$ to $\mathrm{Ba}^{2+}=1.42 \AA$ [53]. A shift of the main reflection peaks to lower angle was observed as the ionic radii increased, which is in good agreement with other studies [31].

An important parameter that can be extracted from the XRD pattern is the degree of Anti-Site Disorder (ASD). This latter value provides informa- 


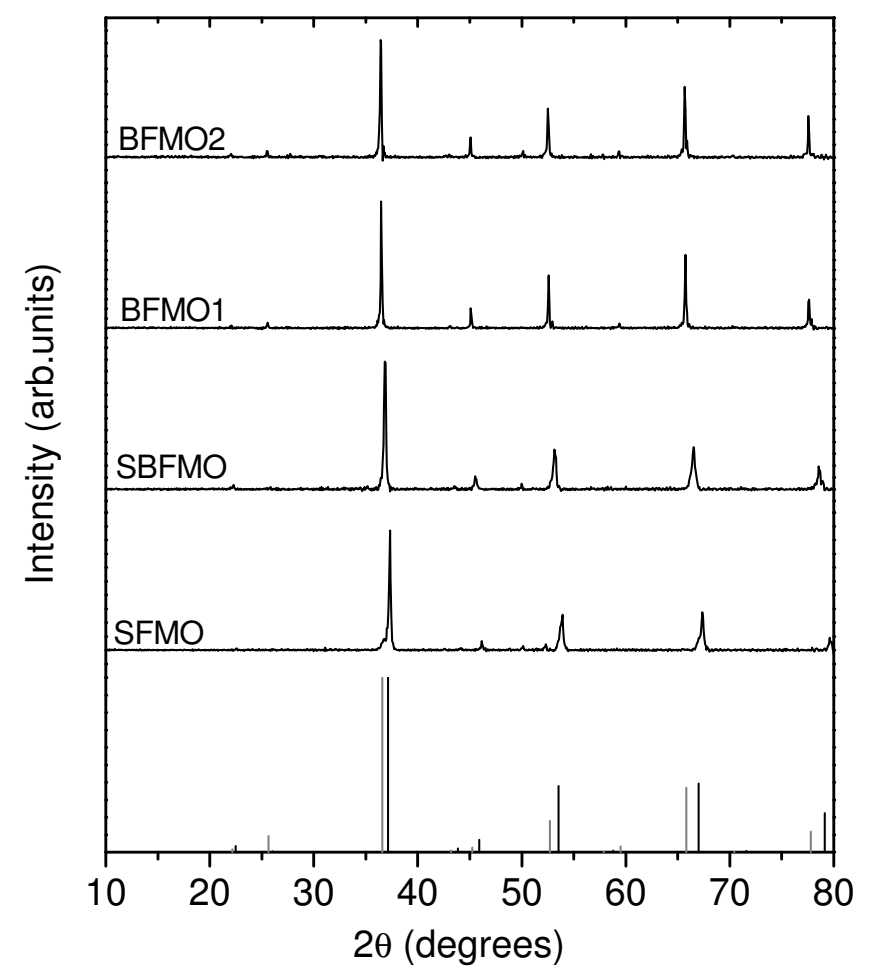

Figure 4.1: XRD patterns of $\mathrm{Sr}_{2-x} \mathrm{Ba}_{x} \mathrm{FeMoO}_{6}$ for $x=0$ (SFMO), $x=1$ (SBFMO) and $x=2$ (BFMO1 and BFMO2) using $C o K_{\alpha}$ radiation. Bottom panel represents the SFMO (black) and BFMO (grey) from the reference patterns.

tion about the disorder from the perfect alternating Fe-Mo ordering [24]. It is crucial to know the degree of Fe-Mo antisite disorder because it is thought to affect the saturation magnetic moment and Curie temperature as well as the magnetoresistance $[15,54,24,55]$. For this reason we measured the ASD fraction from the ratio, $R$ in $\%$, of the (101) peak intensity at $22^{\circ}$ divided by the (200) peak intensity at $37^{\circ}$. Note that the (101) peak referenced to the double perovskite lattice is a superlattice spot in the un- 
derlying conventional perovskite lattice, and it must disappear for a full disordering of Fe and Mo. $\mathrm{Sr}_{2} \mathrm{FeMoO}_{6}$ is cubic above the Curie temperature and the corresponding Miller index for the superlattice peak is (111). As mentioned in the experimental details, a longer scan over 4 hours was performed in the $18^{\circ}$ to $41^{\circ}$ region in order to obtain a better signal to noise ratio.

The relationship between $R$ and the ASD was calibrated by modelling the XRD pattern of $\mathrm{Sr}_{2} \mathrm{FeMoO}_{6}$ using Powder Cell while the ASD was varied from 0 (complete order) to $50 \%$ (completely random Fe-Mo site occupancy). By changing the occupancy of the Fe and Mo atoms in the crystal structure one can observe the drop of the (101) reflection peak versus the increase of the disorder. Note that the (200) reflection does not change with ASD and for this reason it is used as a reference and compared to the (101) peak. Therefore, by using the intensity ratio of these two reflections the ASD can be estimated. The following relationship was derived from these calculations,

$$
R=0.001365(A S D)^{2}-0.136592(A S D)+3.417842
$$

The modelling assumes a random partition of the disorder and this equation can also be used for similar double perovskite structures (e.g. $\mathrm{Ba}_{2} \mathrm{FeMoO}_{6}$ using a scaling factor of 1.72). Using equation 4.1 and the experimental XRD patterns it was found that the ASD did not exceed $12 \%$ in any of the samples (SFMO, SBFMO, BFMO1 and BFMO2). The $\mathrm{Ba}_{2} \mathrm{FeMoO}_{6}$ samples have the lowest amount of ASD with values below $5 \%$.

\subsubsection{Scanning electron microscope images}

Scanning Electron Microscope (SEM) and Energy Dispersive Spectroscopy (EDS) measurements were performed to obtain information about the morphology of the double perovskites. The shape and size of the grains can provide additional information and assist in the understanding of the trans- 
port properties. Many pictures at different magnifications were taken and figure 4.2 represents a typical set from four samples. As seen from this figure, the grain morphologies of the samples are significantly different. The two $\mathrm{Ba}_{2} \mathrm{FeMoO}_{6}$ samples have apparently well-connected grains and a lower concentration of small grains when compared with $\mathrm{SrBaFeMoO}_{6}$ (c) and $\mathrm{Sr}_{2} \mathrm{FeMoO}_{6}(\mathrm{~d})$. EDS measurements were also carried out on these samples and confirmed that the samples were stoichiometric to within 1 $\%$. These observations will be discussed later along with the magnetoresistance analysis.
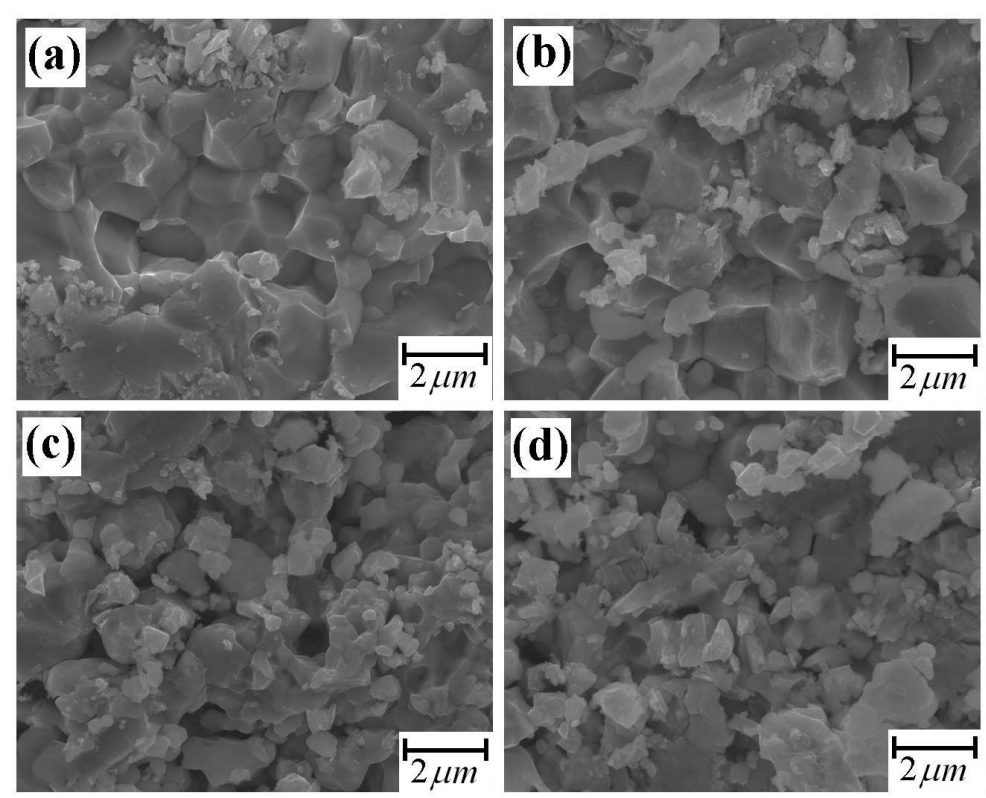

Figure 4.2: Scanning electron microscope images for the (a) $\mathrm{Ba}_{2} \mathrm{FeMoO}_{6}$ (BFMO1), (b) $\mathrm{Ba}_{2} \mathrm{FeMoO}_{6}$ (BFMO2), (c) $\mathrm{SrBaFeMoO}_{6}$ (SBFMO) and (d) $\mathrm{Sr}_{2} \mathrm{FeMoO}_{6}(\mathrm{SFMO})$ samples. 


\subsection{Magnetisation measurements}

\subsubsection{Temperature and field dependence}

Magnetisation measurements were made up to $6 \mathrm{~T}$ and $400 \mathrm{~K}$ using a Superconducting QUantum Interference Devices (SQUID) magnetometer. The data in figures 4.3 and 4.4 are represented by solid lines instead of the

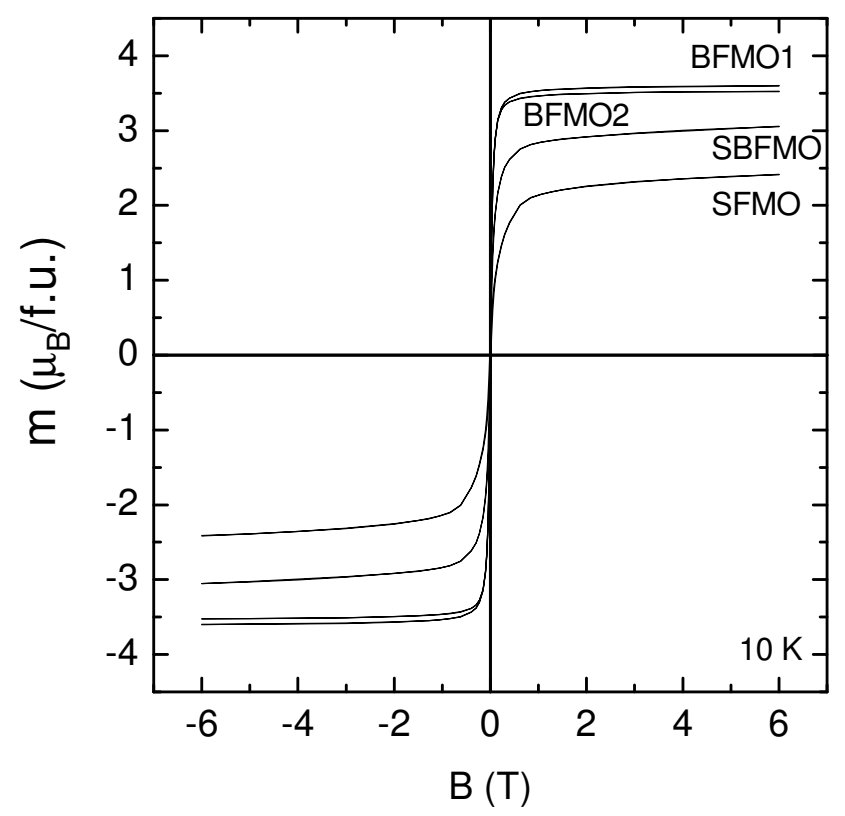

Figure 4.3: Magnetic moment in units of Bohr magnetons, $\mu_{B}$, per formula unit (f.u.) versus the applied magnetic field at $10 \mathrm{~K}$ for the $\mathrm{Sr}_{2-x} \mathrm{Ba}_{x} \mathrm{FeMoO}_{6}$ samples.

data points for clarity. Figure 4.3 shows the magnetic field dependence of the magnetic moment for the various samples. The BFMO samples have a larger saturation magnetic moment $\left(m_{s}\right)$ at around $3.6 \mu_{B} / f . u$. which is close to the expected maximal $4 \mu_{B} / f$.u. in comparison to the two other 
samples (SFMO and SBFMO). As discussed in the introduction, the reduction in the saturation magnetic moment is believed to originate from the increase of the degree of antisite disorder, which is in good agreement with the small degree of ASD found from the BFMO's XRD patterns. We will show in chapter 5 the correlation between the degree of antisite disorder and the saturation magnetic moment.

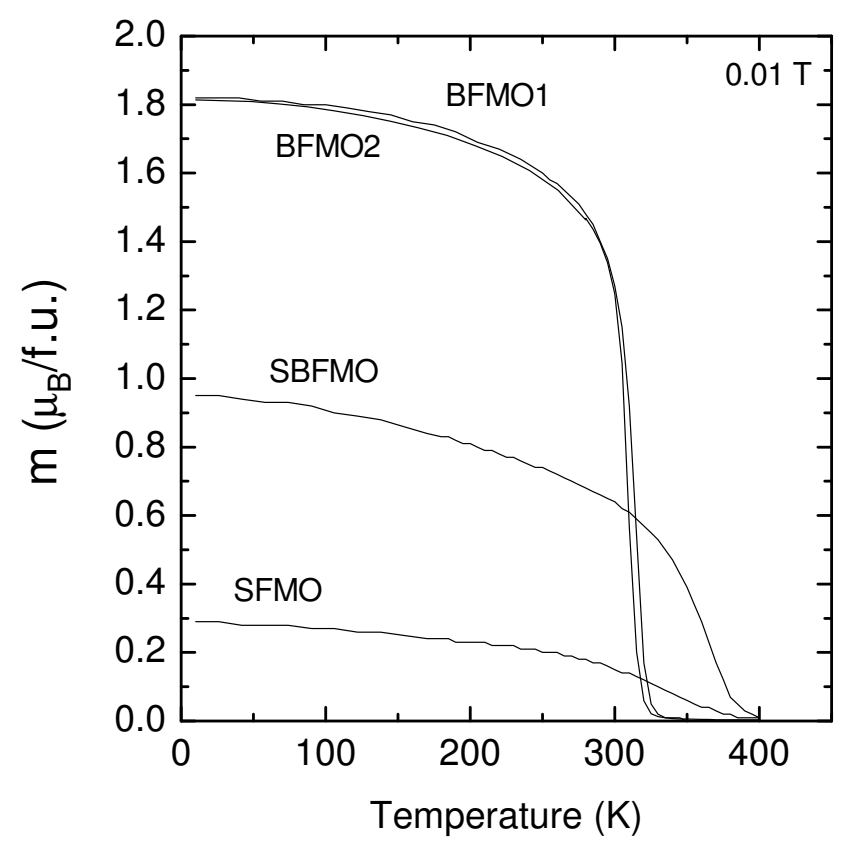

Figure 4.4: Magnetic moment versus temperature at $0.01 \mathrm{~T}$ for $\mathrm{Sr}_{2-x} \mathrm{Ba}_{x} \mathrm{FeMoO}_{6}$ with $x=0$ (SFMO), $x=1$ (SBFMO) and $x=2$ (BFMO1 and BFMO2).

Figure 4.4 presents the magnetic moment versus temperature at 0.01 T. The measurement was carried out at low field as it is known that for small values of the applied magnetic field $m(T)$ shows a sharper drop at 
the Curie temperature $\left(T_{c}\right)$. A temperature increment of $5 \mathrm{~K}$ was adequate for the determination of $T_{c}$. The Curie temperature can be determined by various ways: (i) linear extrapolation of $m(T)$ to zero magnetisation, (ii) the inflection point also called derivative method and (iii) Arrott plots. Arrott plots [56] follow a linear behaviour for $M^{2}$ versus $H / M$ at the Curie temperature (see for example [25]). For low ASD, it seems that (ii) and (iii) give similar $T_{c}$ values [25]. For this reason, the Curie temperature was estimated from the temperature where the derivative of the magnetisation is a maximum negative value as it is the quickest way. From this, we obtained $T_{c}=315 \mathrm{~K}, 365 \mathrm{~K}$, and $\backsim 400 \mathrm{~K}$ for $x=2,1$ and 0 respectively. An identical $T_{c}$ and $m_{s}$ was measured for the two $\mathrm{Ba}_{2} \mathrm{FeMoO}_{6}$ samples. The Curie temperature for the BFMO samples is similar to that reported in other studies [31,57]. From these measurements it is clear that one can select the Curie temperature over a wide temperature range ( $\sim 100 \mathrm{~K}$ ) by inserting various concentrations of $\mathrm{Ba}$ on the strontium site in $\mathrm{Sr}_{2} \mathrm{FeMoO}_{6}$. This type of manipulation could be useful for application purposes.

\subsubsection{Magnetoresistance measurements}

\section{Low field magnetoresistance model}

The magnetoresistance, which is the change of the resistivity under an applied magnetic field, was defined earlier as $M R=\Delta \rho / \rho=[\rho(H)-$ $\rho(0)] / \rho(0)$. It was measured at liquid nitrogen and room temperature for applied magnetic fields up to $0.8 \mathrm{~T}$ using the four terminal method. The maximum MR was found for the BFMO1 sample, which reaches a value of $-25 \%$ for $0.8 \mathrm{~T}$ at $77 \mathrm{~K}$ (see figure $4.5(\mathrm{a})$ ). This can be compared with the $-3.5 \%$ found in the SFMO sample under the same conditions (see figure $4.5(\mathrm{~b}))$. 


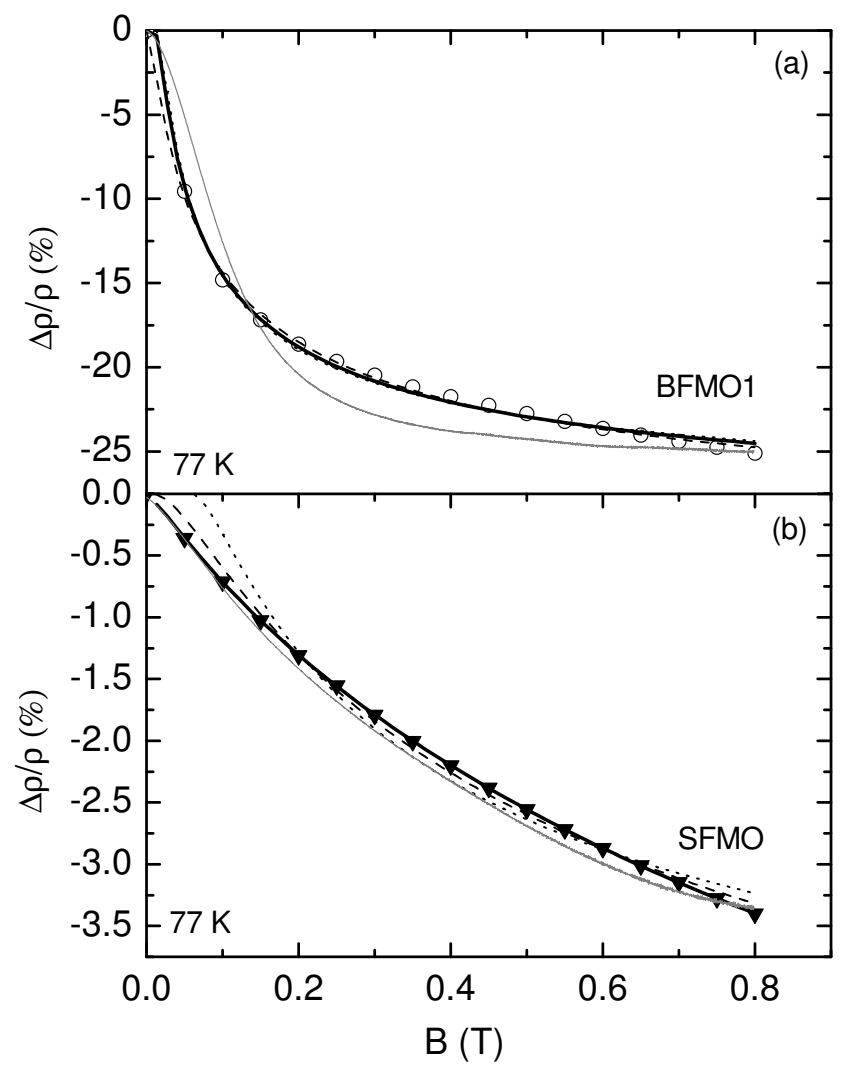

Figure 4.5: Magnetoresistance at $77 \mathrm{~K}$ for (a) BFMO1 (open circles) (b) SFMO (filled down triangles). The curves show fits to the data using equations 4.3 and 4.4 of Serrate et al. (dotted curves), Fisher et al. (dashed curves). The grey curves are fits to the data using equation 4.2 and using the measured bulk reduced magnetic moment. The solid thick lines represent the fit of equations 4.3 and 4.5 . 
The magnetic field dependence of the MR can be understood by noting that the MR in polycrystalline half-metallic double perovskite is believed to be dominated by spin-dependant tunneling across grain boundaries [11, 26]. It can easily be shown that the MR in the case of elastic tunneling across a single barrier and averaged over random grain orientations can be written as $[58,49]$,

$$
\frac{\Delta \rho}{\rho}=\frac{-P^{2} m(H)^{2}}{1+P^{2} m(H)^{2}}
$$

where $P$ is the degree of spin polarisation and it can be written as $P=(n \downarrow$ $-n \uparrow) /(n \downarrow+n \uparrow)$, where $n \downarrow(n \uparrow)$ is the fraction of carriers in the majority (minority) spin state and $m(H)$ is the bulk magnetic moment normalised to its saturation value.

It can be seen in figure 4.5 that using the bulk measured magnetic moment for $m(H)$ (grey curves) provides a poor representation of the data. It is important to note that this model assumes that the regions near the grain boundaries are well-ordered, with a magnetisation characteristic of the bulk, which is unlikely to be true in polycrystalline samples. For this reason Serrate et al. [49] recently argued that a better fit to the MR can be obtain by replacing the bulk magnetic moment, $m(H)$, with the magnetisation from a disordered region near the grain boundaries, $m_{g b}(H)$ guided by the behavior of a spin-glass [49]. Thus, the MR was modelled as [49],

$$
\begin{gathered}
\frac{\Delta \rho}{\rho}=\frac{-P^{2} m_{g b}(H)^{2}}{1+P^{2} m_{g b}(H)^{2}}-\alpha H \\
m_{g b}(H)=\left(1-a / H^{1 / 2}\right)
\end{gathered}
$$

where $H$ is the applied field and $\alpha$ is a parameter that is assumed to be field-independent. The term $\alpha H$ was introduced to account for the intragrain magnetoresistance [49]. It is typically large near the magnetic ordering temperature where the magnetoresistance has been observed to be linear in temperature in other strongly correlated compounds [59]. 
Replacing equation 4.2 with the grain boundary model of Serrate et al. provides a reasonable fit to the BFMO1 data (dotted curve figure 4.5(a)) to obtain the $P$ and $\alpha$ terms but it fails to fit the low-field MR data from the SFMO sample (dotted curve figure 4.5(b)). A better fit can be obtained using the empirical exponential model of Fisher et al. (dashed curves) [60]. This exponential model can be obtained from equation 4.3 using $m_{g b}(H)=$ $\exp \left(-a / H^{1 / 2}\right)$. Note that it reduces to the $m_{g b}(H)$ spin-glass model of Serrate et al. at high fields.

Therefore in order to fit the low and high field magnetoresistance data with a single equation we derived a further improved fit to the low field region and this was obtained using a phenomenological tanh-function correction at low fields,

$$
m_{g b}(H)=\gamma \tanh H / H_{0}+\left[(1-\gamma)-a / H^{1 / 2}\right]
$$

where $\gamma, H_{0}$, and $a$ are fitting parameters. At high fields equation 4.5 will approach $m_{g b}(H)=\left(1-a / H^{1 / 2}\right)$ as found by Serrate et al. At low fields the first term in equation 4.5 accounts for the observed departure from the model of Serrate et al. It can be seen in figure 4.5 that $m_{g b}(H)$ of equation 4.5 provides a good fit to the data (solid curves), and we have used equations 4.3 and 4.5 to fit all of our MR data at $77 \mathrm{~K}$ and $300 \mathrm{~K}$ (figures 4.6 (a) and 4.6(b)). This $m_{g b}(H)$ equation is empirical and was derived in order to obtain a better fit to the low field region.

The resulting values of $P$ at $77 \mathrm{~K}$ are $0.67,0.6,0.6$ and 0.25 for BFMO1, BFMO2, SBFMO and SFMO respectively. The degree of spin polarisation correlates with the maximal magnetoresistance value, which is in agreement with equation 4.2. Note that these values are close to those obtained by fitting the MR data using $m_{g b}(H)=\exp \left(-a / H^{1 / 2}\right)$ and hence the tanh function correctly reproduces the low-field MR without a significant effect on the estimated spin polarisation. As expected, $P$ drops at $300 \mathrm{~K}$ and it was found that $P$ are $0.3,0.19,0.27$ and $\sim 0$ for BFMO1, BFMO2, SBFMO and SFMO respectively. As the magnetoresistance in the double 


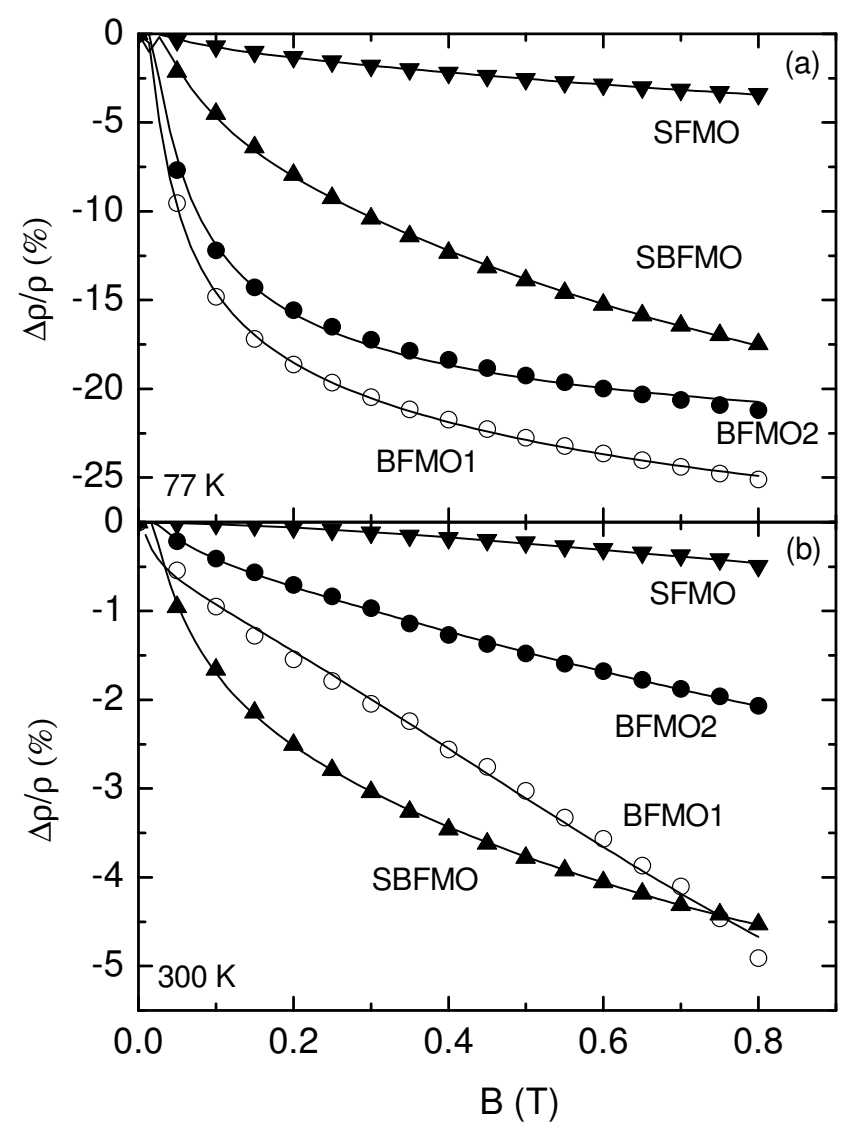

Figure 4.6: Magnetoresistance at (a) $77 \mathrm{~K}$ and (b) $300 \mathrm{~K}$ for SFMO (filled down triangles), SBFMO (filled up triangles), BFMO2 (filled circles) and BFMO1 (open circles). The solid curves are fits to the data using equations 4.3 and 4.5 .

perovskite is expected to be spin-dependent tunneling it would be expected that the small value of $P$ observed for the SFMO sample at $77 \mathrm{~K}$ may be due to a lower $P$ in the inter-grain region close to the grain boundaries. For example, the lower $P$ deduced from equations 4.3 and 4.5 could originate from a wider inter-grain insulating region as well as spin-flip 
scattering from defect states in the inter-grain region. These effects will reduce the total contribution of spin-dependent tunneling to the MR.

Regarding the intra-grain parameter, $\alpha$, it is clear that at low temperatures this parameter is non existent $(\alpha \backsim 0)$ as observed by other investigators [49] and increases with temperature (e.g. BFMO1 $\alpha \backsim 0.0018$ at $300 \mathrm{~K}$ ). As expected it is also greater for sample having the transition temperature close to the measurement temperature i.e. $\mathrm{Ba}_{2} \mathrm{FeMoO}_{6}$ having a $T_{c}=315$ $\mathrm{K}$.

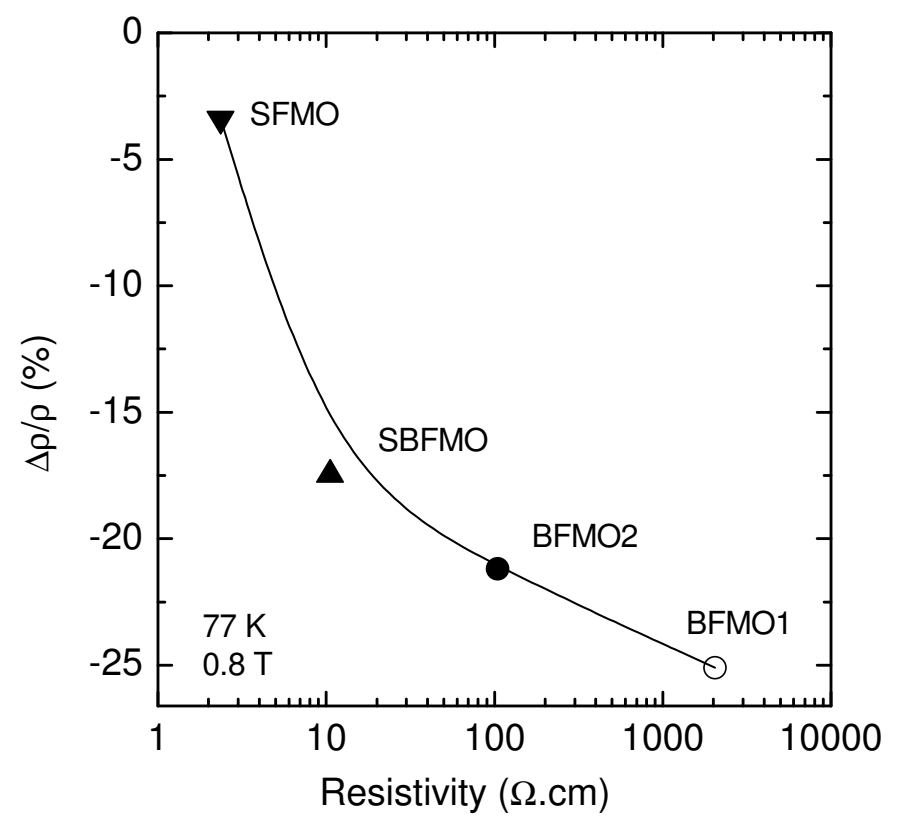

Figure 4.7: Magnetoresistance versus the resistivity at $0.8 \mathrm{~T}$ and $77 \mathrm{~K}$ for SFMO (filled down triangle), SBFMO (filled up triangle), BFMO2 (filled circle) and BFMO1 (open circle). The curve is a guide to the eye. 
The resistivity at $77 \mathrm{~K}$ and $0.8 \mathrm{~T}$ was measured in order to find out if there is any correlation between the value of the MR and the resistivity. As can be seen from figure 4.7, the samples with the largest resistivity at $77 \mathrm{~K}$ also have the highest MR. They also have apparently better connected grains and a lower concentration of small grains when compared with the other two samples (see figure 4.2). This has been observed by our group previously in two $\mathrm{Ba}_{2} \mathrm{FeMoO}_{6}$ samples where the sample with well-connected grains had a large resistivity [45]. The inter-grain regions are too small to be seen in the SEM images but it is likely that the high MR occurs for samples where $P$ in the disordered inter-grain region is not significantly reduced from that in the bulk and the insulating inter-grain barrier widths are optimised. Thus, maximizing the MR in polycrystalline samples requires the optimisation of the inter-granular insulating region as well as minimizing the disorder near the grain boundaries.

\section{High field magnetoresistance measurements}

Some high field measurements up to $12 \mathrm{~T}$ down to liquid helium were performed at the École Polytechnique Fédérale de Lausanne (EPLF) in Switzerland. The magnetoresistance of the pure compound $\left(\mathrm{Sr}_{2} \mathrm{FeMoO}_{6}\right)$ was measured at $4.2 \mathrm{~K}(\mathrm{a})$ and $300 \mathrm{~K}(\mathrm{~b})$ and is plotted in figure 4.8. The data were fitted to the model of Serrate et al. (dashed curves) and our empirical formula (solid curves). From both models, a similar degree of spin polarisation was obtained with $P=0.65$ and $P=0.18$ for $4.2 \mathrm{~K}$ and $300 \mathrm{~K}$ respectively. It is also interesting to mention that the MR data at $300 \mathrm{~K}$ in figure 4.8(b) shows a nearly linear behaviour versus the applied magnetic field up to $12 \mathrm{~T}$. As mentioned previously, this behaviour was observed in other compounds when the measurement temperature gets closer to $T_{c}[49,57]$. 


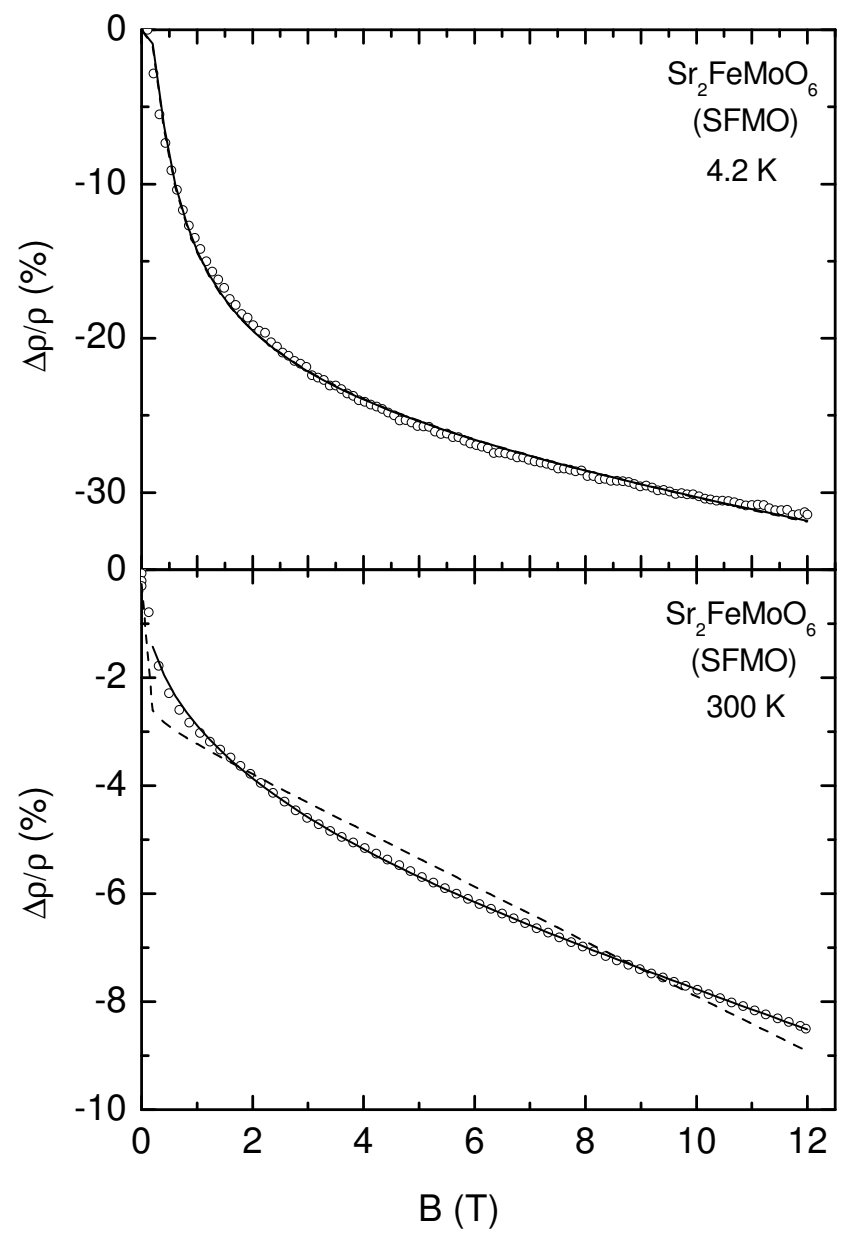

Figure 4.8: Magnetoresistance at (a) $4.2 \mathrm{~K}$ and (b) $300 \mathrm{~K}$ versus the applied field, $B$, for the pure compound $\mathrm{Sr}_{2} \mathrm{FeMoO}_{6}$. Also shown are fits to the data for the models of Serrate et al. (dashed curve) and our empirical formula (solid curve). 
Now moving to $\mathrm{Ba}_{2} \mathrm{FeMoO}_{6}$, it was shown that there is a magnetoresistance of $-25 \%$ at $77 \mathrm{~K}$ at $0.8 \mathrm{~T}$ for the BFMO1 sample (figure $4.6(\mathrm{a})$ ) which is large for double perovskites. Therefore, it would be of interest to measure the MR of this sample under a larger field and lower temperature. Figure 4.9 presents the results of the measurement at $4.2 \mathrm{~K}$ and up to $12 \mathrm{~T}$ and with the fit of the empirical formula developed in the previous section.

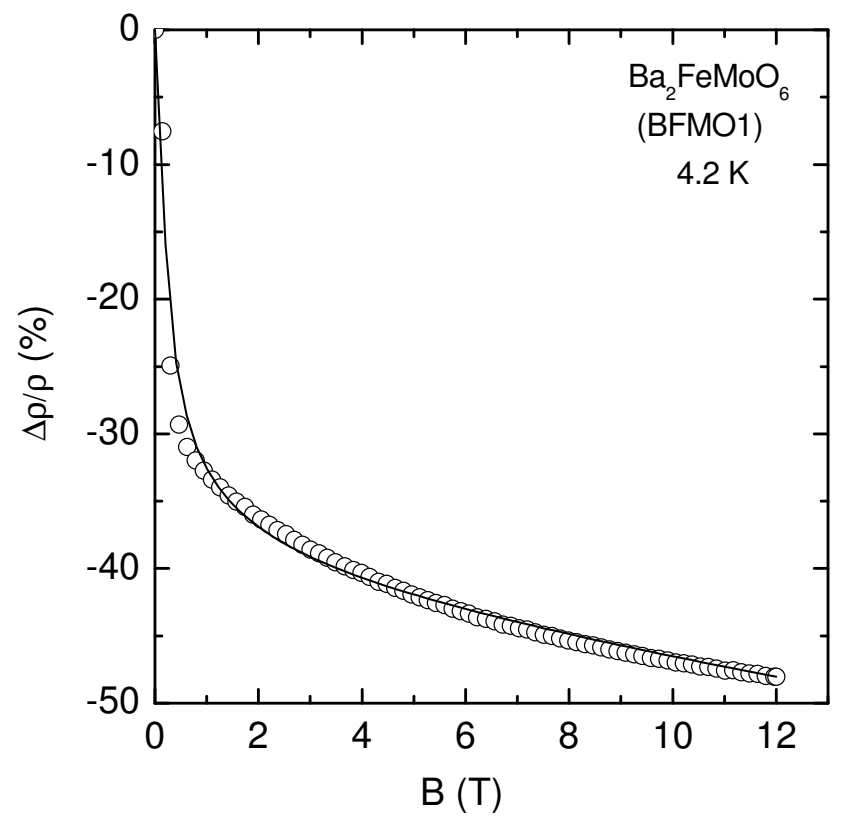

Figure 4.9: Magnetoresistance of $\mathrm{Ba}_{2} \mathrm{FeMoO}_{6}$ versus the applied magnetic field, $B$ in Tesla, at $4.2 \mathrm{~K}$. The solid line is the best fit of equations 4.3 and 4.5 .

From the fit, a $P=0.85$ was obtained which is close to the maximal value of $P$ (i.e. $P=1$ ) that is expected in the spin-dependent tunneling magnetoresistance model for polycrystalline samples. We previously found that the degree of spin polarisation correlated with the magnetoresistance 
(figure 4.6) that is again proved here where the magnetoresistance reached a value as large as $-48 \%$, which is close to the maximal $-50 \%$. All this seems to indicate that this sample is close to the "ideal" compound for application purposes where the inter-granular region providing the spintunneling transport possesses the adequate properties to maximize the magnetoresistance.

\subsection{Summary}

Structural, magnetic and transport properties of $\mathrm{Sr}_{2-x} \mathrm{Ba}_{x} \mathrm{FeMoO}_{6}$ polycrystalline samples were studied. A large low field MR was found for $x=$ 2 and it was shown that there is a correlation between the resistivity and the low field MR. Furthermore, the low field MR can be fitted to the intergrain model of Serrate et al. where the tunneling MR is limited by a disordered region near the insulating grain boundaries. Thus, it is the electronic properties near the grain boundary that lead to the large low field MR. An empirical formula was developed to obtain a better fit in the low-field region in comparison with the available models. High field magnetoresistance measurements were performed and validate our model up to $12 \mathrm{~T}$. In addition, a magnetoresistance as large as $-48 \%$ was measured at $12 \mathrm{~T}$ and $4.2 \mathrm{~K}$ that is close the maximum $-50 \%$ expected in the spin-dependent tunneling magnetoresistance model for polycrystalline samples. 


\section{Chapter 5}

\section{Electron doping by lanthanum in $\mathbf{S r}_{2-x} \mathbf{L a}_{x} \mathbf{F e M o O}_{6}$}

The effect of electronic doping on the magnetic and electronic properties is important in furthering the understanding of $\mathrm{Sr}_{2} \mathrm{FeMoO}_{6}$ double perovskites. Lanthanum substitution on the strontium site is a way to electron-dope the system.

Previous studies have shown that electronic doping leads to an increase in the Curie temperature $[15,16]$ and photoemission spectroscopy data have been interpreted in terms of an increasing density of states at the Fermi level [61]. Recently, Alonso et al. argued that without the structural changes, non-stoichiometry, or antisite disorder the Curie temperature should decrease with electron doping [62]. Serrate et al. attempted to diminish these structural effects by inserting various amount of $\mathrm{Ba}$ and La onto the Sr site but still found an increase in $T_{c}$ with electron doping, which supported the band filling scenario [63]. This latter has also been observed in a different double perovskite, $\mathrm{Nd}_{x} \mathrm{Ca}_{2-x} \mathrm{FeMoO}_{6}$, where the Curie temperature was raised due to the $\mathrm{Nd}^{3+}$ insertion onto the divalent $\mathrm{Ca}^{2+}$ site $[16,64]$. Other techniques such as nuclear magnetic resonance [65] and/or Mössbauer spectroscopy [66] have been used to show that electron doping indeed produces a carrier injection in the $4 d(\mathrm{Mo})$ 
states.

Surprisingly, no thermoelectric power measurements, which have been revealed to be very useful in the study of other strongly correlated electron systems, have been performed on a series of electron doped samples. For this reason, we have performed magnetic, transport and structural measurements on $\mathrm{Sr}_{2-x} \mathrm{La}_{x} \mathrm{FeMoO}_{6}(x=0$ to 0.5$)$ and report the results in this chapter. Two papers reporting these results were published in Physical Review B [67] and Journal of Magnetism and Magnetic Materials [52].

\subsection{X-ray diffraction patterns}

The room temperature XRD data from the various $\mathrm{Sr}_{2-x} \mathrm{La}_{x} \mathrm{FeMoO}_{6}$ samples are plotted in figure 5.1. They were normalised to the same (200) peak intensity. The XRD data show single-phase material indexed to a tetragonal structure (I4/mmm space group) with $a=5.59 \AA$ and $c=7.90 \AA$. There is no evidence for a detectible change in the lattice parameters with Lasubstitution. Evidently, the small reduction in the ionic radii (1.26 $\AA$ for $\mathrm{Sr}^{2+}$ and $1.16 \AA$ for $\mathrm{La}^{3+}$ ) [53] with a maximal substitution of $25 \%$ La for $\mathrm{Sr}$ is not sufficient to change the lattice parameters by an amount that is detectible using our XRD set-up.

As discussed in section 4.1, the degree of ASD is a crucial parameter in the analysis of the magnetisation and transport data. For this reason, the ASD of all the La-doped samples were determined. The (101) peak can be seen more clearly in the insets to figure 5.1 where it has been scaled by a factor of 150 and vertically offset. It can be seen that this latter peak decreases versus the La-concentration, which suggests that the amount of antisite disorder increases. For a more accurate evaluation of the ASD we used equation 4.1 and found values of 21, 23, 26, 26, 29 and $36 \%$ for $x=0$, $0.1,0.2,0.3,0.4$ and 0.5 respectively. These values will be discussed later with the magnetisation data. 


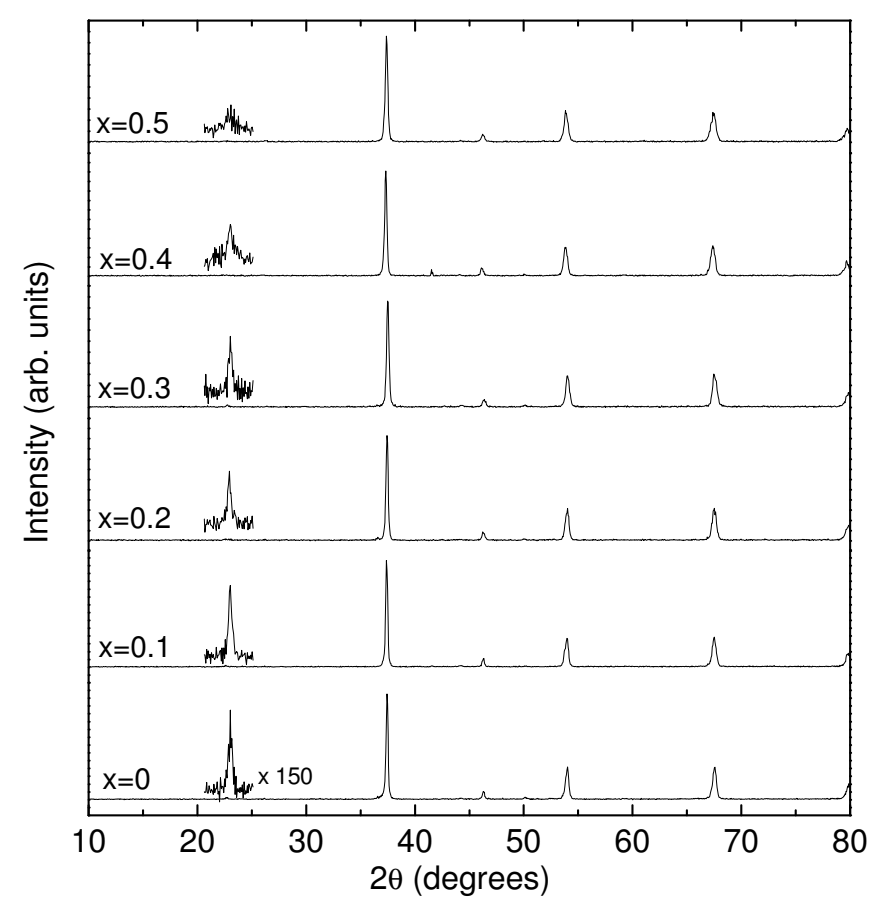

Figure 5.1: XRD patterns of $\mathrm{Sr}_{2-x} \mathrm{La}_{x} \mathrm{FeMoO}_{6}$ using $C o K_{\alpha}$ radiation. The insets show the expanded (101) peak that is also vertically offset.

\subsection{Transport properties in La-doped double per- ovskite}

\subsubsection{Temperature dependence of the resistivity}

The zero-field resistivity down to liquid helium temperature is plotted in figure 5.2. All the samples show a small increase in the resistivity with decreasing temperature. There is an initial decrease in the magnitude of the resistivity and then a smaller decrease with increasing La concentration. The magnitude of the resistivity for the pure compound is comparable to 


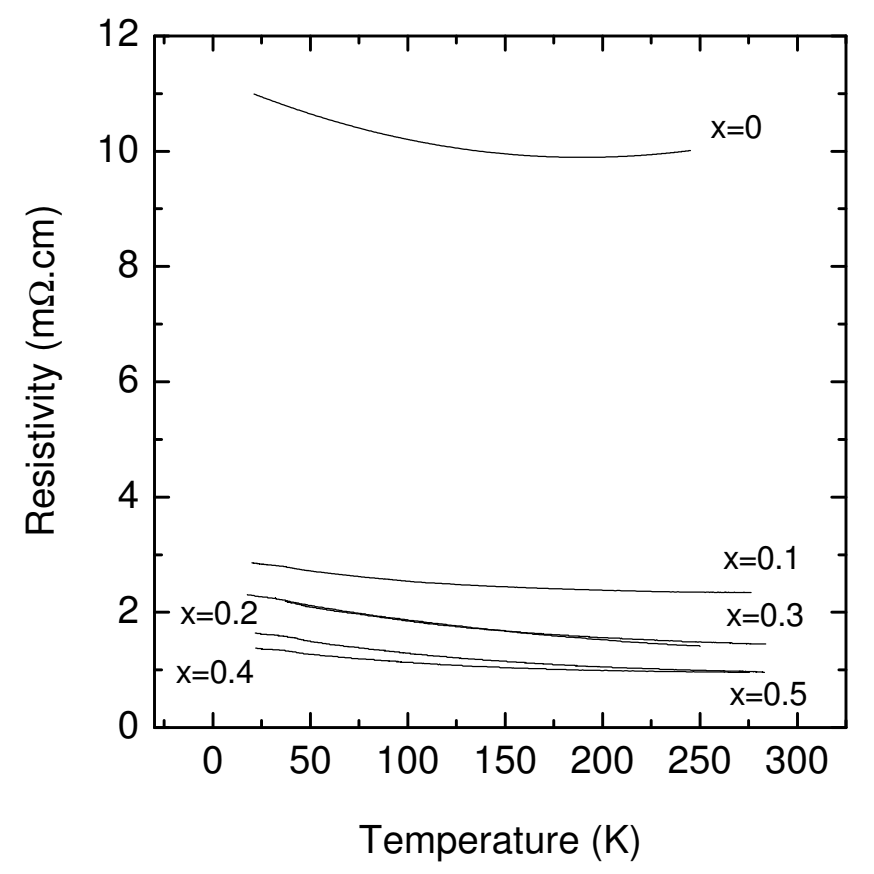

Figure 5.2: Resistivity versus temperature of the $\mathrm{Sr}_{2-x} \mathrm{La}_{x} \mathrm{FeMoO}_{6}$ samples.

that reported in other studies but it is one order of magnitude greater than in single crystals [68]. This again suggests that the resistivity is dominated by the resistance across the grain boundaries (chapter 4 ). The higher resistivity found in our pure sample may be due to a grain morphology that is different from that in the La substituted samples. There is a general trend in the resistivity where the resistivity is lowest in the more La substituted samples. As shown before, the changes in the resistivity are mainly driven by the inter-granular rather than intrinsic properties. Therefore it is possible that the small changes in the resistivity in La-doped sample $(x=0.1$ to $x=0.5$ ) originates from the electron doping while the order of magnitude drop between $x=0$ and $x=0.1$ is likely to be due to small changes 
in the grain boundary morphology that lead to reduced tunneling barriers across the grain boundaries and hence better connectivity. Now looking at the ASD, the lowest resistivities are observed in the samples with the highest ASD. This result is unexpected as one can think that the disorder will increase the spin-scattering and hence the resistivity. However, the situation is rather complex as the increase in ASD will lead to nanoscale clusters of $\mathrm{SrFeO}_{3-\delta}$ and $\mathrm{SrMoO}_{3}$. We will see for instance in chapter 7 that depending on the oxygen content, $\mathrm{SrFeO}_{3-\delta}$ could be either metallic or insulating while $\mathrm{SrMoO}_{3}$ is insulating. It has also been shown that the oxygen content in $\mathrm{Sr}_{2} \mathrm{FeMoO}_{6+\delta}$ is playing a major role in the resistivity [69]. There are clearly too many parameters to draw a definite conclusion on these resistivity measurements.

\subsubsection{Thermoelectric power of $\mathrm{Sr}_{2-x} \mathrm{La}_{x} \mathrm{FeMoO}_{6}$}

The thermoelectric power of all $x$-concentrations was measured as a function of temperature using the standard temperature differential technique. It should be recalled that unlike the resistivity, the thermoelectric power of these polycrystalline ceramics is not affected by high inter-grain boundary resistance. It is affected by inter-grain boundaries only if they develop a large thermal resistance because the thermoelectric response is driven by the temperature gradient rather than by the electric field. A systematic increase in the thermoelectric power with increasing La-concentration was found as can be seen in figure 5.3. The data can be regarded as representing the thermoelectric power of the majority, intra-grain material. The low temperature peak and negative high temperature gradient for high Laconcentrations are similar to that reported by Fisher et al. [30] from thermoelectric power measurements on $\mathrm{Sr}_{1.5} \mathrm{La}_{0.5} \mathrm{FeMoO}_{6}$.

The temperature dependence of the thermoelectric power is remarkably similar to that found in the superconducting cuprates [70]. Furthermore, both classes of compounds show a systematic increase in the room 


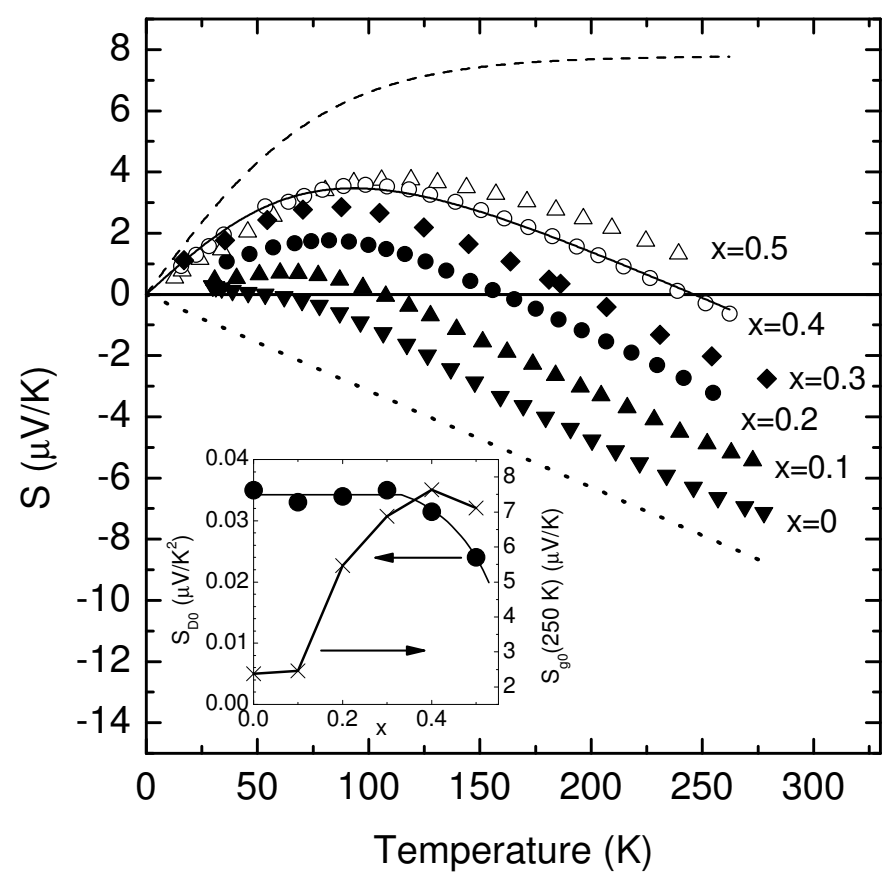

Figure 5.3: Thermoelectric power versus temperature for $\mathrm{Sr}_{2-x} \mathrm{La}_{x} \mathrm{FeMoO}_{6}$ samples with $x=0$ (filled down triangles), $x=0.1$ (filled up triangles), $x=$ 0.2 (filled circles), $x=0.3$ (filled diamonds), $x=0.4$ (open circles) and $x=0.5$ (open up triangles). The solid curve is a simulation of the $x=0.4$ data with a diffusion term (dotted curve) and a phonon-drag term (dashed curve). Inset: plot of the diffusion thermoelectric power prefactor (filled circles, left axis) and the phonon drag thermoelectric power prefactor (crosses, right axis). The solid curve is a guide to the eye.

temperature thermoelectric power with increasing electron doping. In the case of the superconducting cuprates, this correlation has proved to be a useful tool to estimate the doping state [71]. The close correspondence between the SFMO and superconducting cuprate thermopower suggests a 
common mechanism in both systems even though the Fermi surfaces are different and the electronic state is three dimensional for SFMO and two dimensional for the superconducting cuprates.

In order to understand the thermoelectric power data one must note that the thermoelectric power has both a "diffusion" component $S_{d}$ associated with only the electron gas and its dispersion, and a "phonon drag" component $S_{g}$ arising from momentum delivered to the electrons when they scatter from phonons, and is thus related to the phonon dispersion and the electron-phonon interaction. In the simplest scenario these simply add, so that the thermopower can be written as,

$$
S(T)=S_{d}(T)+S_{g}(T)
$$

In the relaxation time approximation the diffusion thermoelectric power in a cubic material can be written as [72],

$$
S_{d}(T)=-\frac{1}{e T \sigma} \int(\epsilon-\mu)\left(\frac{\partial f}{\partial \epsilon}\right) \sigma(\epsilon)
$$

where

$$
\sigma(\epsilon)=\left(\frac{e^{2}}{\Omega_{0}}\right) \sum_{\mathbf{k}} \nu(\mathbf{k})^{2} \tau(\mathbf{k}) \delta(\epsilon(\mathbf{k})-\epsilon)
$$

where $\epsilon$ is the energy, $\mu$ is the chemical potential, $f$ is the Fermi function, $\Omega_{0}$ is the normalised volume, $\nu$ is the quasiparticle velocity and $\tau(\mathbf{k})$ is the scattering time. The dependence predicted by equation 5.2 is simply proportional to temperature if the Density Of States (DOS) is effectively constant within $k T$ of the Fermi energy, but clearly very rapid changes in the DOS near the Fermi energy can lead to more complex temperature dependencies to $S_{d}(T)$.

Although $S(T)$ could be modelled by assuming electron doping in the proximity to a sharp peak in the DOS [73], that proposal would require structure within less than $\sim 10 \mathrm{meV}$ of the Fermi energy. This structure would need to remain that close even as the doping is changed by the 
introduction of as much as $0.5 \mathrm{La}$ per formula unit, which appears to be highly unrealistic and not supported by band structure calculations [74]. In fact band structure calculations show an increasing density of states for up to $\backsim 300 \mathrm{meV}$ below and above the Fermi energy in the pure compound. Thus, assuming that the entire temperature dependence of $S(T)$ is due only to the proximity to a sharp peak in the DOS is untenable if it is to model the persistence of the systematic pattern over the large doping range in the present studies.

A more natural interpretation of $S(T)$ is available if phonon drag is included in the model, which has proved useful in interpreting the thermopower from the superconducting cuprates [70]. The drag component is expected to approximately mirror the phonon specific heat, rising at low temperature as phonons become thermally excited and saturating above the Debye temperature, $\Theta_{D}$. In conventional metals the effect is attenuated at high temperatures where phonon-phonon scattering begins to dominate over phonon-electron scattering, which then reduces the phonon-electron momentum exchange and leads to a $1 / T$ dependence. On the assumption that phonon-electron scattering remains strong up to ambient temperature [70], the phonon drag contribution is approximately proportional to the Debye function, rising from zero at zero temperature and saturating above $\Theta_{D}$. Within this model the prediction is for a high temperature thermoelectric power that consists of a constant phonon drag component and a diffusion component that is linear with temperature [70]. As the temperature is reduced the drag component falls and the total thermopower approaches zero at zero temperature, as required by the third law of thermodynamics. Within this model the high temperature slope gives the diffusion term and the extrapolation of that slope to zero temperature is the saturated drag component [70].

Given the remarkable similarity between the thermopower in SFMO and the superconducting cuprates, the thermoelectric power data was analysed in terms of the same phonon drag model. In the present work a 
phenomenological expression was chosen for the drag component and a temperature-linear diffusion component as $S(T)=S_{g 0} \tanh \left(T / T_{0}\right)-S_{D 0} T$. The resulting $S_{g}(T)$ (dashed curve), $S_{d}(T)$ (dotted curve) and $S(T)$ (solid curve) are plotted in figure 5.3 for $x=0.4$.

The values of $S_{g 0}$ (crosses) and $S_{D 0}$ (filled circles) are plotted in the inset to figure 5.3. It can be seen that $S_{D 0}$ is independent of $x$ for $x$ as high as 0.3 and decreases for higher La-concentrations. This appears to suggest that doping effects on $S_{d}(T)$ are small for as much as 0.3 doped electrons per Mo if all the doped electrons appear in the down-spin conduction band with $\mathrm{Fe}\left(\mathrm{t}_{2 g}\right)$ and $\mathrm{Mo}\left(\mathrm{t}_{2 g}\right)$ character. We also find that $S_{g 0}$ increases for $x$ $>0.2$ and appears to saturate for $x>0.4$. These results are understood by noting that the diffusion component depends primarily on the energy scale over which the DOS varies, evaluated at the Fermi energy. The small decrease in $S_{D 0}$ observed for $x=0.5$ is consistent with electron doping and an increasing Fermi energy based on band structure calculations and assuming a rigid band model [74]. In contrast, the drag component is a very sensitive function of the balance between normal and umklapp scattering events, and that balance is strongly influenced by changes to the Fermi surface $[70,75]$.

\subsection{Magnetisation measurements}

\subsubsection{Antisite disorder effect on the saturation magnetic mo- ment}

Magnetisation measurements under an applied magnetic field of up to $6 \mathrm{~T}$ at $10 \mathrm{~K}$ were made on all the La-doped samples. It can be seen in figure 5.4 that there is a systematic decrease in the high field magnetisation with increasing La-concentration. It has been suggested that this decrease is due to ASD as well as doping effects [15]. This model assumes static moments on the Fe and Mo sites as well as a Fe-Mo superexchange mechanism that 


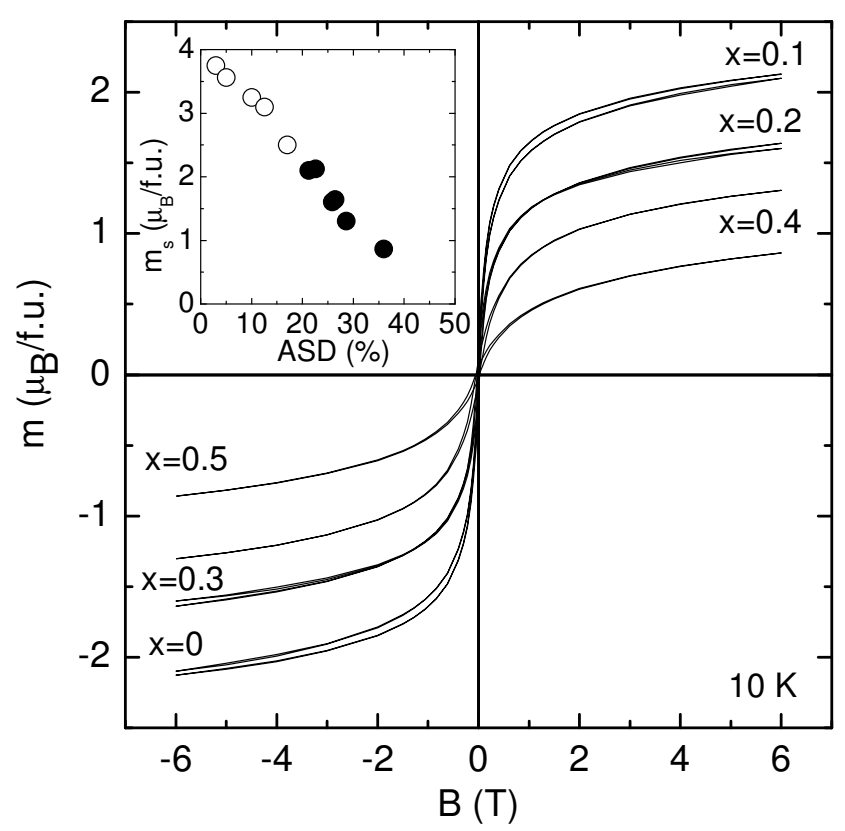

Figure 5.4: Magnetic moment curves at $10 \mathrm{~K}$ for $\mathrm{Sr}_{2-x} \mathrm{La}_{x} \mathrm{FeMoO}_{6}$. Inset: plot of the magnetic moment at $6 \mathrm{~T}$ versus the ASD for this study (filled circles) and from Balcells et al. [54] for $x=0$ (open circles).

leads to the magnetically ordered state. It also assumes that some of the electrons introduced by La-doping appear on the Fe site. The situation is possibly more complicated as we have mentioned earlier. For example it has been suggested that Fe should remain in the 3+ state [74] and there is no well-defined Mo moment of $1 \mu_{B}$ per formula unit since for the pure compound the electron in the originally down-spin $\mathrm{Mo}\left(t_{2 g}\right)$ orbital is itinerant [19].

The decrease in the high field magnetisation with increasing La-doping can be primarily attributed to ASD, which is apparent in the inset of figure 5.4 (filled circles). In order to derive a relationship between the ASD and 
$m_{s}$ over the whole range of disorder i.e. 0 to $50 \%$ the data by Balcells et al. [54] (open circles) were used for low degree of antisite disorder (ASD < $20 \%$ ). Our data is just a continuation of that found in the pure $\mathrm{Sr}_{2} \mathrm{FeMoO}_{6}$ compound and we found that $m_{s}=4.0-9(A S D)$. Consequently, by extrapolation we expect the magnetic order to disappear for ASD $=44 \%$, which is slightly less than the assumed $50 \%$ [15].

\subsubsection{Shift of the Curie temperature upon La-doping}

Similar to a previous study [15], the Curie temperature $\left(T_{c}\right)$, estimated from the maximum negative gradient of the magnetisation data, increases with increasing La-concentration. The magnetic moment at $6 \mathrm{~T}$ up to 700 $\mathrm{K}$ is plotted in figure 5.5 and we found that the Curie temperature is 390 $\pm 5 \mathrm{~K}, 390 \pm 5 \mathrm{~K}, 395 \pm 10 \mathrm{~K}, 395 \pm 10 \mathrm{~K}, 405 \pm 15 \mathrm{~K}$ and $415 \pm 15 \mathrm{~K}$ for $x$ $=0,0.1,0.2,0.3,0.4$ and 0.5 respectively.

We have also fitted the low temperature magnetic moment data to [76],

$$
m(T)=m_{s}\left(1-a_{0} T^{n}\right)
$$

where $a_{0}$ is the spin-wave dispersion, $m$ the spontaneous magnetic moment and $m_{s}$ is the saturation magnetic moment. This equation describes the drop of the spontaneous magnetic moment within a domain as the temperature increases in a ferromagnet. We found that $n=1.5 \pm 0.1$ for all La-concentrations, which is expected for a 3D Heisenberg ferromagnet. This is also known as the Bloch $T^{3 / 2}$ law that describes the low temperature thermal demagnetisation. Surprisingly, $a_{0}$ is only weakly dependent on La-concentration as can be seen in the inset to figure 5.5. Since $a_{0}$ is affected by the spin-wave dispersion, this result suggests that there is no significant change in the spin-wave dispersion for small wave-vectors even though there is a large change in $m_{s}$. 


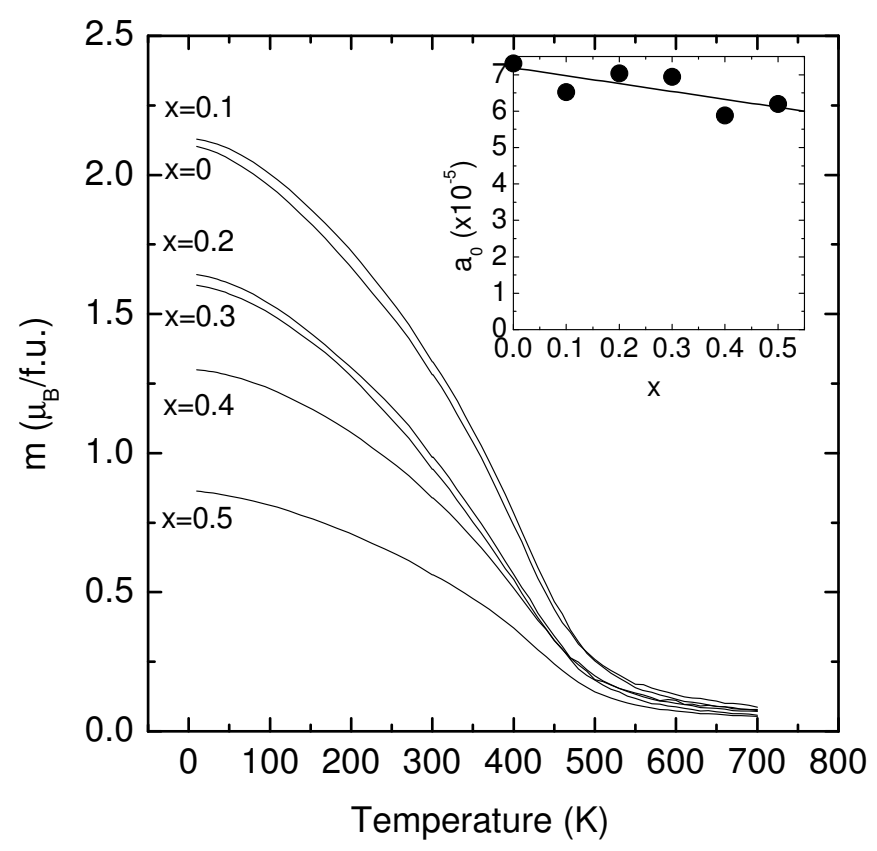

Figure 5.5: Magnetic moment versus temperature at $6 \mathrm{~T}$ for $\mathrm{Sr}_{2-x} \mathrm{La}_{x} \mathrm{FeMoO}_{6}$. Inset: plot of prefactor for the low temperature fit to the data as described in the text. The solid line is a linear best fit to the data.

\subsection{Magnetoresistance in La-doped samples}

The magnetoresistance at $77 \mathrm{~K}$ versus the applied magnetic field was measured up to $0.8 \mathrm{~T}$ for three La-concentrations as can be seen from figure 5.6. The decreasing MR at $0.8 \mathrm{~T}$ with $x$ is likely to be due to the increase in the amount of antisite disorder (ASD) and/or change in the grain morphologies rather than an electron doping effect. It was shown in chapter 4 that there is a clear correlation between the resistivity and the magnetoresistance. However, in the case of the La-doped samples, the resistivity does not change greatly from $x=0.1$ to $x=0.5$ and therefore could not explain 
the observed reduction of the MR. On the other hand, the ASD changes with the La-concentration with an ASD $=21,26$ and $36 \%$ for $x=0,0.2$ and 0.5 respectively. A correlation between the MR and the ASD has already been observed in samples where the grain boundaries insulating barrier were weak and hence having small resistivities [26]. Furthermore, it was

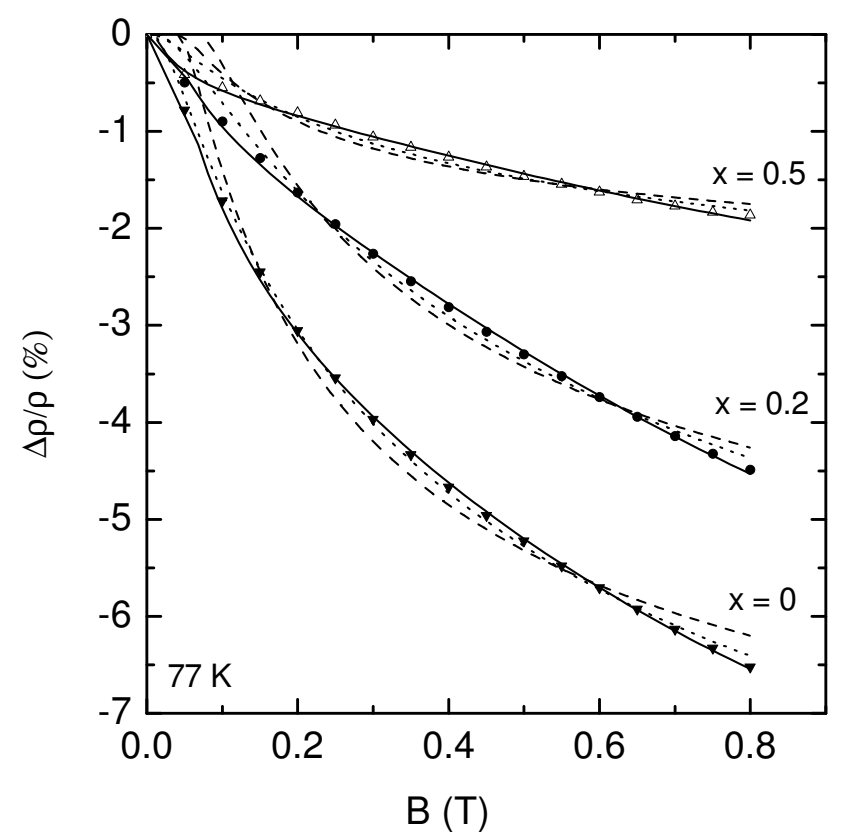

Figure 5.6: Magnetoresistance at $77 \mathrm{~K}$ versus applied magnetic field, B, for $\mathrm{Sr}_{2-x} \mathrm{La}_{x} \mathrm{FeMoO}_{6}$ with $x=0,0.2$ and 0.5. Also shown are fits to the data for the models of Serrate et al. (dashed curved), Fisher et al. (dotted curve) and our model (solid curve). For the details of the model please refer to chapter 4 .

also found in chapter 4 that the grain morphologies changed with the Baconcentration and this could also occur for the La-doped samples. This will certainly affect the magnetoresistance. Therefore it seems possible 
that the grain morphologies together with the ASD could explain the drop of the magnetoresistance. However, there is certainly too many parameters to draw clear conclusions from this result. Further research should be performed, such as making La-samples having a similar ASD, in order to understand all the contributions to the magnetoresistance.

We have used the three magnetoresistance models explained in section 4.1 to obtain the degree of spin polarisation, $P$ (see figure 5.6). Clearly the two models of Serrate et al. and Fisher et al. do not provide the best fit of the data at low field and therefore our model was used to determine the degree of spin polarisation with $P=0.33,0.28$ and 0.17 for $x=0,0.2$ and 0.5 respectively. It is again evident that the degree of spin polarisation correlates with the maximal magnetoresistance values as observed in the $\mathrm{Sr}_{2-x} \mathrm{Ba}_{x} \mathrm{FeMoO}_{6}$ samples.

\subsection{Summary}

The $\mathrm{Sr}_{2-x} \mathrm{La}_{x} \mathrm{FeMoO}_{6}$ thermoelectric power displays a temperature dependence and shift with electron doping that is remarkably similar to that observed in the superconducting cuprates. We have modelled the Lainduced changes in terms of electronic doping that affects the diffusion and the phonon-drag components. The decrease in the magnetisation at $6 \mathrm{~T}$ with increasing La-doping has been shown to be predominantly due to ASD. The high field magnetisation is extrapolated to be zero at $\backsim 44$ $\%$ ASD, which is slightly less than the $50 \%$ or completely random FeMo site occupancy. Finally, it was found that the magnetoresistance in the La-doped samples correlate well with the the degree of antisite disorder. This could imply that the disorder is more likely to occur at the grain boundaries as the transport properties are dominated by spin-dependent tunneling. 


\section{Chapter 6}

\section{Magnetic and electronic properties of $\mathbf{S r}_{2} \mathbf{F e}_{1-x} \mathbf{A l}_{x} \mathbf{M o O}_{6}$}

There have been a number of reports of partial or complete substitutions onto the $\mathrm{Sr}, \mathrm{Fe}$ or Mo sites in $\mathrm{Sr}_{2} \mathrm{FeMoO}_{6}$ with the aim to further understand the physics of these strongly correlated compounds $[15,61,67,77$, $78,79]$. Fe site substitutions are particularly interesting because the exchange splitting of the $\mathrm{Fe}\left(t_{2 g}\right)$ and $\left(e_{g}\right)$ orbitals is central in determining the magnetic and electronic states.

Previous substitutions for Fe have mainly focussed on $\mathrm{Cr}, \mathrm{Mn}$ and $\mathrm{Mg}$ but there is as yet no clear pattern concerning how they affect $T_{c}$ or the saturation magnetic moment. For example, reports on $\mathrm{Cr}$ substitution for $\mathrm{Fe}$ in $\mathrm{Sr}_{2} \mathrm{Fe}_{1-x} \mathrm{Cr}_{x} \mathrm{MoO}_{6}[80,81,82,83]$ show a large drop in the saturation magnetic moment per formula unit. The end member, $\mathrm{Sr}_{2} \mathrm{CrMoO}_{6}$, is also believed to be half-metallic with a $T_{c}$ of $450 \mathrm{~K}$ [84] and a smaller moment that is expected in view of the smaller $\mathrm{Cr}^{3+}$ moment when compared with the $\mathrm{Fe}^{3+}$ moment. However, the drop found in $\mathrm{Sr}_{2} \mathrm{Fe}_{1-x} \mathrm{Cr}_{x} \mathrm{MoO}_{6}$ is substantially more rapid than can be explained by the difference between the ionic moments. It has been suggested that the increase of the amount of antisite disorder could trigger a change in the exchange interaction and therefore explain this faster decrease in the saturation moment $[81,82,85]$. 
Interestingly, Mn substitution for Fe also leads to a large drop in the saturation moment. In this case $T_{c}$ falls as well, and the magnetic order is destroyed completely for $x>0.7$ in $\mathrm{Sr}_{2} \mathrm{Fe}_{0.3} \mathrm{Mn}_{0.7} \mathrm{MoO}_{6}$ [86]. The end member, $\mathrm{Sr}_{2} \mathrm{MnMoO}_{6}$, is an antiferromagnetic insulator with a Néel temperature of $\sim 12 \mathrm{~K}$ [87], which might suggest that Mn is being substituted as $\mathrm{Mn}^{2+}$. However, $\mathrm{Mn}^{3+}$ does occur in other half-metallic double perovskites (e.g. $\mathrm{Sr}_{2} \mathrm{MnReO}_{6}$ with $T_{c} \backsim 120 \mathrm{~K}$ ) [88], so it is unclear whether Mn substitutes as $\mathrm{Mn}^{2+}$ or $\mathrm{Mn}^{3+}$ in $\mathrm{Sr}_{2} \mathrm{Fe}_{1-x} \mathrm{Mn}_{x} \mathrm{MoO}_{6}$.

$\mathrm{Mg}^{2+}$ substitution for $\mathrm{Fe}^{3+}$ has the effect of reducing both the average exchange between magnetic Fe ions and doping the material with holes, which would be expected to further affect exchange within carriermediated models. In line with this expectation a study of $\mathrm{Mg}$-substituted $\mathrm{Sr}_{2} \mathrm{Fe}_{1-x} \mathrm{Mg}_{x} \mathrm{MoO}_{6}$ showed a large decrease in both the saturation moment and $T_{c}[89]$.

A better understanding of the effect of Fe-site substitutions on the magnetic and electronic properties can be obtained by substitutions that are expected to be isoelectronic. The convention assumed in band structure calculations is that the iron ions are in the $\mathrm{Fe}^{3+}$ state, though there is experimental evidence that the Fe valence might be close to 2.5+ [29]. Aluminium substitution is a good candidate but has not been studied extensively in the double perovskite [90]. In addition, $\mathrm{Al}^{3+}$ is non magnetic and therefore could provide interesting behaviour as it would be expected to dilute the magnetic system. With this in mind we have prepared $\mathrm{Sr}_{2} \mathrm{Fe}_{1-x} \mathrm{Al}_{x} \mathrm{MoO}_{6}(0 \leq x \leq 0.3)$ samples substituting $\mathrm{Al}^{3+}$ on the Fe sites and performed structural, transport and magnetic measurements. We report our results in this chapter and a paper has been published in Physica $\mathrm{B}[85]$. 


\subsection{Single phase materials $\mathrm{Sr}_{2} \mathrm{Fe}_{1-x} \mathrm{Al}_{x} \mathrm{MoO}_{6}$}

XRD measurements show that all the $\mathrm{Sr}_{2} \mathrm{Fe}_{1-x} \mathrm{Al}_{x} \mathrm{MoO}_{6}$ samples are single phase and there is no evidence for minority phases within the limit of detectibility by XRD $(\sim 1 \%)$.

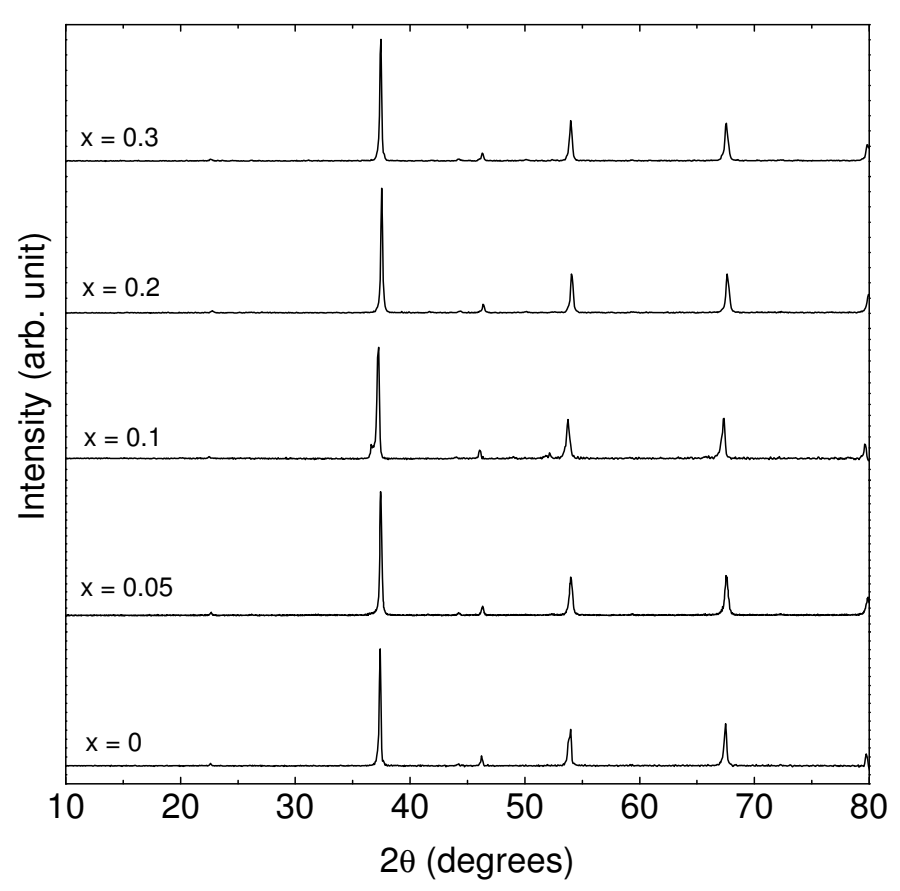

Figure 6.1: XRD patterns of $\mathrm{Sr}_{2} \mathrm{Fe}_{1-x} \mathrm{Al}_{x} \mathrm{MoO}_{6}$ for $x=0,0.05,0.1,0.2$ and 0.3 .

The partial substitution of $\mathrm{Al}$ on the Fe site makes it difficult to obtain accurate values for the amount of Fe-Mo and Al-Mo antisite disorder as done previously (chapter 4 and 5). We cannot determine if it is the Fe or the $\mathrm{Al}$, which is going to be disordered with the Mo. In order to have a rough indication of the ASD in these Al-samples, we assumed that only the Fe 
was preferentially disordered with the Mo. It was found that the ASD is 17 $\%$ for the pure sample and no more than $25 \%$ for the $x=0.3$ sample. We show later that the saturation magnetic moment does not drop substantially with Al-concentration and therefore this is in good agreement with a small change in the ASD.

\subsection{Transport properties of $\mathbf{S r}_{2} \mathbf{F e}_{1-x} \mathbf{A l}_{x} \mathbf{M o O}_{6}$}

\subsubsection{Resistivity measurements}

The resistivity down to liquid helium temperature was measured for four of the compounds (see figure 6.2). The $x=0.1$ sample broke and therefore

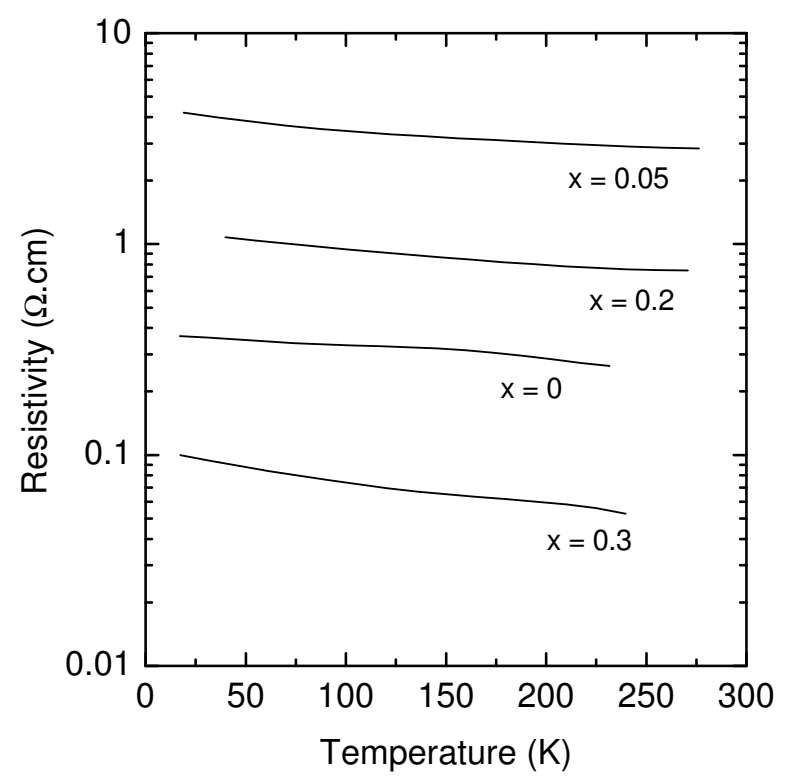

Figure 6.2: Temperature dependence of the resistivity for $\mathrm{Sr}_{2} \mathrm{Fe}_{1-x} \mathrm{Al}_{x} \mathrm{MoO}_{6}$ for $x=0,0.05,0.2$ and 0.3 . 
no resistivity measurement could be performed. The missing data did not interfere with the interpretation of the results. All the samples exhibit a small increase in the resistivity with decreasing temperature and the resistivity ranges between $\backsim 0.05$ and $5 \Omega$.cm, without any systematic variation with $\mathrm{Al}$ concentration. Thus, even for up to $0.3 \mathrm{Al}, \mathrm{Sr}_{2} \mathrm{Fe}_{1-x} \mathrm{Al}_{x} \mathrm{MoO}_{6}$ can be interpreted as being metallic, with the conductivity limited by insulating grain boundaries.

\subsubsection{Thermoelectric power}

Information about the intra-grain region can be obtained from the thermoelectric power (TEP), which is not affected by high inter-grain electrical resistances. TEP measurements were made on all samples. For clarity the data for only $x=0$ and $x=0.3$ was plotted in figure 6.3. We have decomposed these results into their diffusion and phonon drag components as $S(T)=S_{d}(T)+S_{g}(T)$. The fitting of the data was done in a similar way to that in chapter 5 using the equation,

$$
S(T)=S_{g 0} \tanh T / T_{0}-S_{d 0} T
$$

The fits were done on all the $x$-concentration samples and only $x=0$ and $x=0.3$ samples are shown in figure 6.3 by the solid and dashed curves respectively. It can be seen in the inset to figure 6.3 that $\mathrm{Al}$ substitution leads to a systematic decrease in $S_{d 0}$ obtained by fitting all the TEP data, suggesting an increasing $E_{F}$ and carrier concentration with the introduction of $\mathrm{Al}$. This would not be expected if $\mathrm{Al}^{3+}$ substitutes for $\mathrm{Fe}^{3+}$, supporting the conclusions based on Mössbauer measurements that have been interpreted in terms of $\mathrm{Fe}^{2.5+}$ [29]. The partial substitution of $\mathrm{Fe}^{2.5+}$ by $\mathrm{Al}^{3+}$ can then lead to a decrease of the magnitude of the diffusion thermoelectric power related to an increased carrier density in the spin-down conduction band. 


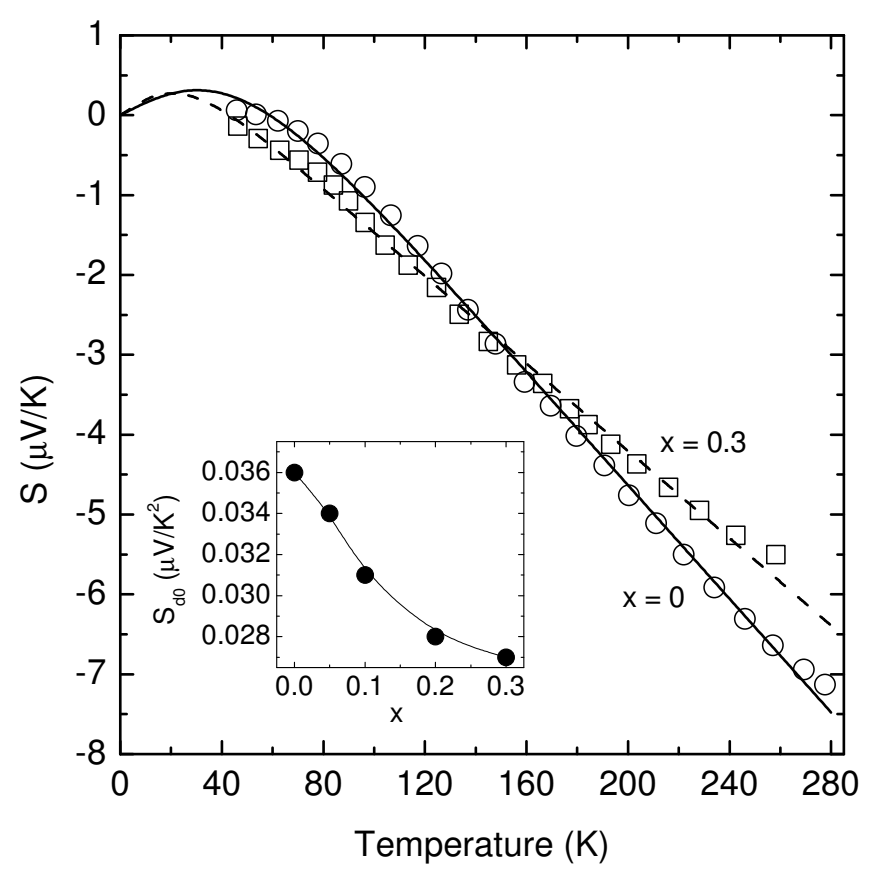

Figure 6.3: Thermoelectric power versus temperature for $\mathrm{Sr}_{2} \mathrm{Fe}_{1-x} \mathrm{Al}_{x} \mathrm{MoO}_{6}$ samples with $x=0$ (open circles) and $x=0.3$ (open squares). The two curves are the fits of equation 6.1. Inset: plot of the diffusion thermopower prefactor $S_{d 0}$ versus $\mathrm{Al}$ concentration for all the samples. The line is a guide for the eye.

\subsection{Magnetic response of $\mathrm{Sr}_{2} \mathrm{Fe}_{1-x} \mathrm{Al}_{x} \mathbf{M o O}_{6}$}

\subsubsection{Temperature and field dependence of the magnetic moment}

In figure 6.4(a) the magnetic moment, in Bohr magnetons per formula unit, against the applied magnetic field at $10 \mathrm{~K}$ is plotted. 

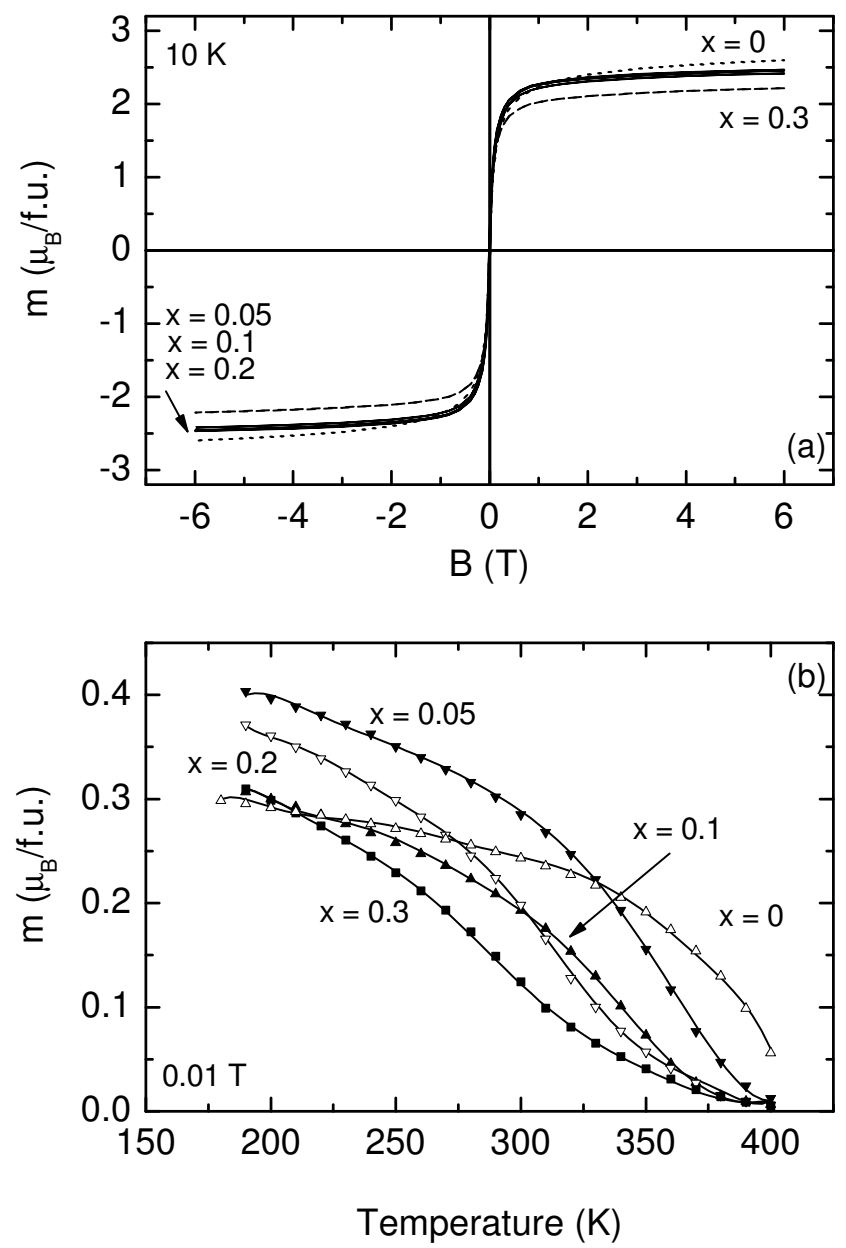

Figure 6.4: (a) Magnetic moment per f.u. versus applied magnetic field at $10 \mathrm{~K}$ for all the $\mathrm{Sr}_{2} \mathrm{Fe}_{1-x} \mathrm{Al}_{x} \mathrm{MoO}_{6}$ samples. The $x=0.05,0.1$ and 0.2 data nearly fall on the same curve and they are plotted as thick solid lines. The $x=0$ data are plotted as a dotted curve and the $x=0.3$ data are plotted as a dashed curve. (b) Magnetic moment per f.u. versus temperature at 0.01 $\mathrm{T}$, where the $\mathrm{Al}$ concentrations are indicated on the figure. 
It can be seen that there are no large changes in the saturation moment with increasing $\mathrm{Al}$ concentration; we return to this observation later. In contrast, the Curie temperatures, estimated from the maximum negative gradients in figure 6.4(b) and shown in figure 6.5 and table I, show a clear systematic variation. The decrease of $114 \mathrm{~K}$ for $x=0.3$ is substantially larger than the $50 \mathrm{~K}$ reported earlier [90].

\begin{tabular}{cccccc}
\hline \hline $\mathrm{x}$ & 0 & 0.05 & 0.1 & 0.2 & 0.3 \\
\hline$\rho(\Omega . \mathrm{cm})$ at $100 \mathrm{~K}$ & 0.3 & 3.3 & - & 0.9 & 0.07 \\
$T_{c}(\mathrm{~K})$ & 400 & 363 & 341 & 314 & 286 \\
$m_{s}\left(\mu_{B} / f . u.\right)$ & 2.60 & 2.46 & 2.41 & 2.47 & 2.21 \\
$\Delta \rho / \rho(\%)$ at $0.8 \mathrm{~T}$ and $77 \mathrm{~K}$ & -4.93 & -5.19 & -3.60 & -7.07 & -6.17 \\
$P$ at $77 \mathrm{~K}$ & 0.276 & 0.294 & 0.242 & 0.339 & 0.315 \\
\hline \hline
\end{tabular}

Table 6.1: Extracted parameters from the magnetic and transport measurements in $\mathrm{Sr}_{2} \mathrm{Fe}_{1-x} \mathrm{Al}_{x} \mathrm{MoO}_{6}$ with $x=0,0.05,0.1,0.2$ and 0.3 .

Figure 6.5 shows $T_{c}$ versus the Al concentration (filled circle) compared with several models: a mean-field random-site dilution model (solid line) and two percolation models [91]. At higher concentrations one expects there to be a percolation threshold, which for a 3D simple cubic lattice with strictly nearest-neighbour interactions occurs near $x=0.69$ [92]. The same percolation threshold exists for the 3D Heisenberg and Ising models, but the dependence on $x$ below the percolation threshold is different in the $S=\frac{1}{2}$ 3D Heisenberg (dotted curve) and Ising (dashed curve) random site dilution models $[91,93]$. In our case the data is close to that expected for 3D mean-field or Ising random site dilution. As we could not make single phase material for concentrations greater than $x=0.3$ we looked in the literature for data above this $x$-concentration. However this could not be found for the Al-substitution and for this reason the Mn-substitution data was used. $T_{c}$ versus the Mn concentration of Moritomo et al. [86] is plotted in figure 6.5 (open circle). It is clear that the Mn substitution is different from the aluminium one as the manganese is magnetic and therefore may 
participate in the exchange interaction between the orbitals and not act as a non-magnetic dilution. Despite this, it seems that $T_{c}$ of $\mathrm{Sr}_{2} \mathrm{Fe}_{1-x} \mathrm{Mn}_{x} \mathrm{MoO}_{6}$ follows the 3D Ising model and possess a sudden suppression of $T_{c}$ for $x$ $=0.7$ which is exactly what would be expected in the two $3 \mathrm{D}$ models.

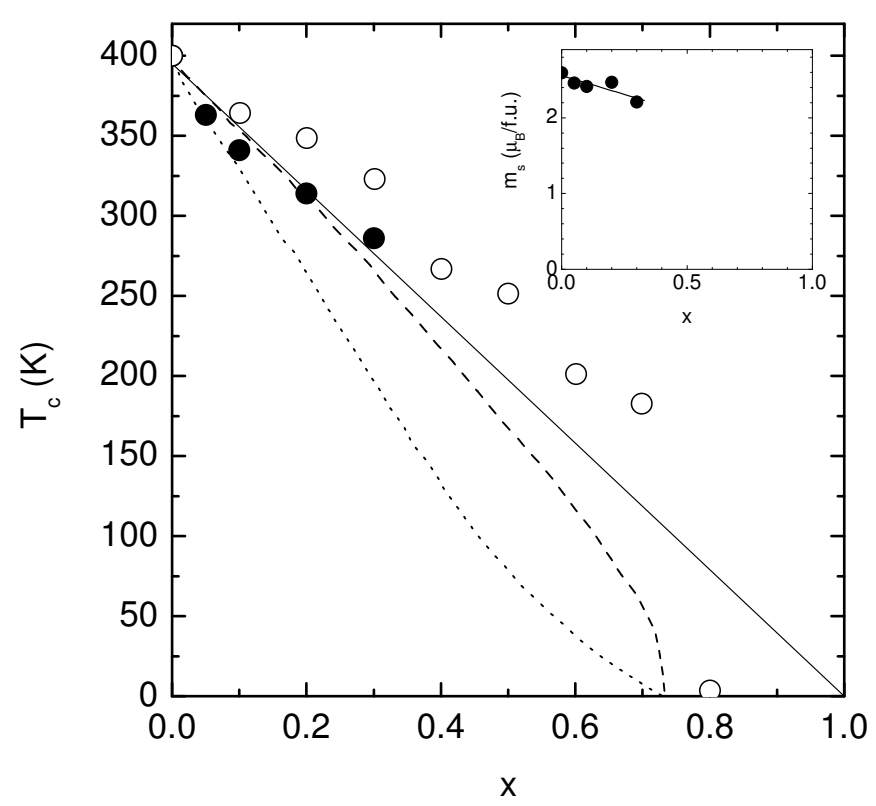

Figure 6.5: Plot of $T_{c}$ against the $\mathrm{Al}$ fraction (filled circle) and the $\mathrm{Mn}$ substituted data of Moritomo et al. [86] (open circle). Also shown are curves for random site dilution in a mean field (solid curve), 3D Ising (dashed curve), and 3D Heisenberg model (dotted curve) [91]. Inset: plot of the saturation magnetic moment at $6 \mathrm{~T}$ against the $\mathrm{Al}$ fraction. The solid curve is a linear fit to the data.

It was previously found, from the thermoelectric power measurement, that the increasing Al-substitution suggests an electron doping. It should be noted that it could lead to an additional increase in $T_{c}$ that might be 
expected in the kinetically driven model of Sarma et al., which is supported by magnetisation [94] and Zero Field Nuclear Magnetic Resonance (ZFNMR) [65] measurements. Thus, in a simple model,

$$
T_{c}(x)=g(x)\left[T_{c}(0)+\Delta T(x)\right]
$$

where $g(x)$ is the 3D random site dilution function and $\Delta T(x)$ is the additional increase in $T_{c}$ induced by electron doping. Therefore, it is also possible that the data could be described by the 3D Heisenberg random site dilution model and electron doping that leads to an increase in $T_{c}(0)$. However, theoretical calculations would be required to make a rigorous differentiation among the two percolation models.

Returning to the inset of figure 6.5 it can be seen that the saturation moment decreases with increasing $\mathrm{Al}$ concentration. In the simplest model it might be expected that,

$$
m_{s}=(1-x)\left[m_{F e}-m_{M o}\right]
$$

where $m$ is the magnetic moment in $\mu_{B} / f . u ., x$ is the concentration of the substituent, $m_{F e}$ and $m_{M o}$ is the moment on the Fe and Mo site respectively. This equation is limited to small $x$ and the assumption that the Mo moment is orientated opposite to that of the Fe moment. It is difficult to estimate the size of the Mo moment and various studies report absolute values between 0 and $1 \mu_{B} / f . u$. [94, 95, 96]. Even with an assumed zero moment for Mo the above equation predicts a $30 \%$ drop in the moment per f.u. in our most concentrated sample, but it was found that $m$ decreases by only $0.39 \mu_{B} / f . u$. instead of the predicted $0.78 \mu_{B} / f . u$. As shown previously, the degree of antisite disorder is playing a role in the decrease of the saturation magnetic moment and in the case of the Al-samples it is difficult to provide an exact value for this quantity. However, the drop of $m_{s}$ is smaller than expected and thus it seems that the ASD is not playing a major role otherwise the decrease would be more important. We demonstrate below that zero field nuclear magnetic resonance measurements im- 
ply that most of this decrease can be attributed to Fe; the Mo moment is nearly independent of $x$ at least for $x$ up to 0.2 .

\subsubsection{Magnetoresistance}

Turning next to the conducting properties of the samples, it must be recognised that the situation is complicated in the presence of grain-boundary tunneling. The difficulty in controlling the grain boundary properties has already been noted in chapter 4 , and it is signalled here by the fact that the resistivity at $100 \mathrm{~K}$ varies non-systematically with $\mathrm{Al}$ concentration (see table I). Nonetheless it is exactly this grain boundary tunneling that leads ultimately to the high magnetoresistance. The source of the high MR is that inter-grain tunneling is enhanced when the spin of the carriers in the two grains are aligned. For temperatures far below $T_{c}$, the MR in polycrystalline samples is dominated by spin-dependent tunneling across the grain boundaries and the absolute value can approach $-50 \%$ at high fields for close to full electronic spin polarisation (see chapter 4 ).

It is apparent in figure 6.6(a) that the magnitude of the $77 \mathrm{~K}$ magnetoresistance for our samples does not change systematically with increasing Al substitution, nor does it bear a simple relationship with the resistivity. As mentioned earlier the MR can be affected by disorder near the grain boundaries, insulating regions between the grains that are too thick for spin-dependent tunneling, and spin-flip scattering within the boundaries, and that all of these affect the resistivity as well. The granular nature of the polycrystalline samples means that the conduction process resembles that of a 3D resistor network in which the resistors depend in a complex way on the relative polarisation in the two neighbouring grains. In the present case at $77 \mathrm{~K}$, a significant fraction of the grain boundaries are not optimizsed for spin-dependent tunneling, so that the MR is low and there is no correlation with the resistivity or the $\mathrm{Al}$ concentration. Using the magnetoresistance model developed in chapter 4 we determined the de- 


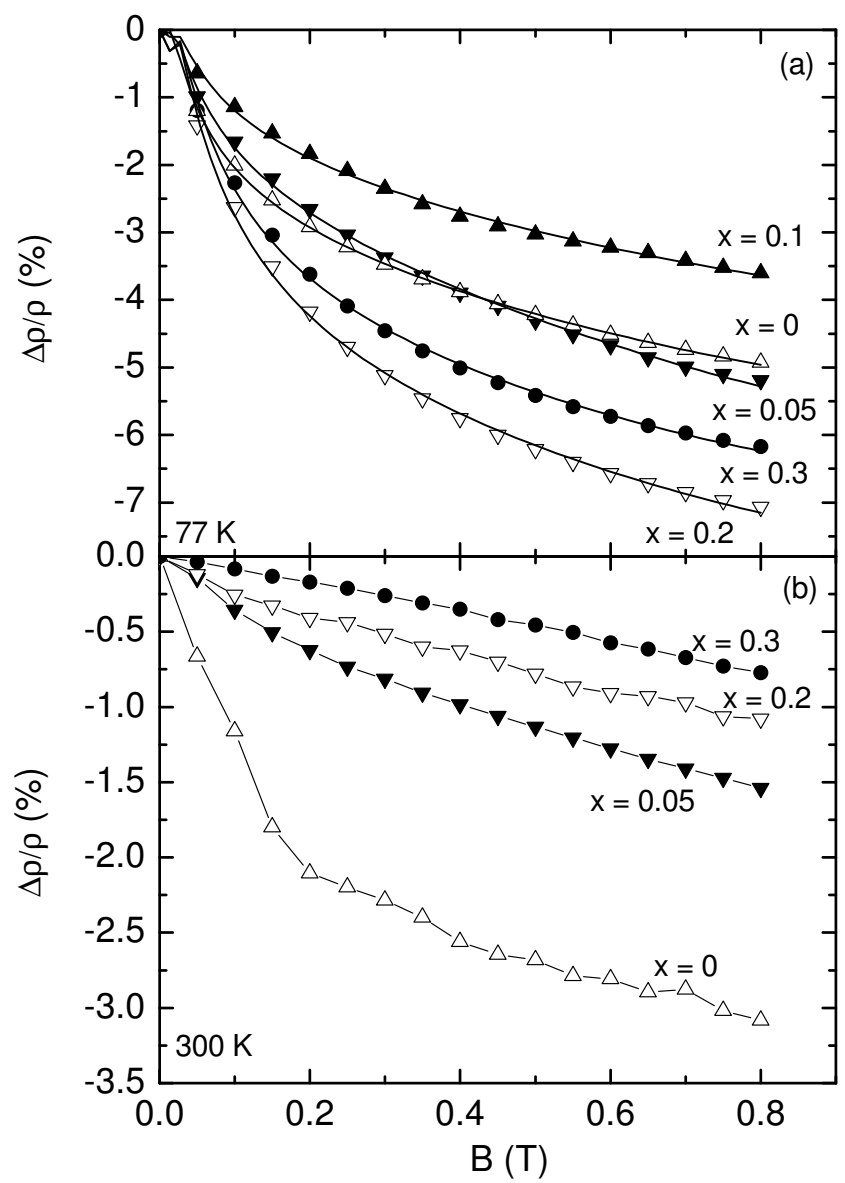

Figure 6.6: (a) Plot of the magnetoresistance at $77 \mathrm{~K}$ against the applied field, $B$, for the various $x$-concentration in $\mathrm{Sr}_{2} \mathrm{Fe}_{1-x} \mathrm{Al}_{x} \mathrm{MoO}_{6}$ with $x=0$ (open up triangles), $x=0.05$ (filled down triangles), $x=0.1$ (filled up triangles), $x=0.2$ (open down triangles) and $x=0.3$ (filled circles). The solid line is a fit to the data using equations 4.3 and 4.5. (b) Plot of magnetoresistance at $300 \mathrm{~K}$ against the applied field, $B$, for various $\mathrm{Al}$ concentrations. The $x=0.1$ was not measured as the bar-shape sample broke. 
gree of spin polarisation and present it in table I.

It can be seen in figure 6.6(b) that, contrary to the situation at low temperature, the $\mathrm{MR}$ at $300 \mathrm{~K}$ does correlate with the $\mathrm{Al}$ concentration. This is likely to be due to the systematic decrease in $T_{c}$ with increasing $\mathrm{Al}$ concentration. For example, $T_{c}$ has reduced to $285 \mathrm{~K}$ for $x=0.3$ and the MR is nearly linear in temperature, which has been observed in similar and other compounds close to $T_{c}[49,59,57]$.

\subsubsection{Zero Field Nuclear Magnetic Resonance (ZFNMR)}

Zero field nuclear magnetic resonance measurements were performed by Dr Grant Williams at the Institut Für Weltwirtschaft (IFW) in Dresden, Germany. These measurements were carried out to complement the Alsubstituted magnetisation data in order to probe the change in the Mo magnetic moment. This also provides a measure of the spin-wave dispersion and the $n$ parameter in the Bloch's law. ZFNMR were made using a Hahn pulse sequence and a $\pi / 2$ pulse width of $1 \mu$ s for frequencies from $50 \mathrm{MHz}$ to $70 \mathrm{MHz}$, which was the tuning range of the NMR coil. The spectra were obtained from the spin-echo peak height and using the pointby-point method. The usual $\omega^{2}$ correction was applied [97].

The results are shown in figure 6.7 for $x=0$ (figure 6.7(a)) and $x=$ 0.2 (figure 6.7(b)). The full ZFNMR spectra for the pure as well as Lasubstituted samples have already been reported by other researchers [65], where the main peak was attributed to hyperfine coupling from a spin on the Mo site to the Mo nucleus $[95,98]$. Thus the resonance frequency, $F$, can be written as $F=\left(\gamma_{n} / 2 \pi\right) A\left(m_{M o} \mu_{B}\right)$ where $\gamma_{n}$ is the Mo nuclear gyromagnetic ratio, and $A$ is the hyperfine coupling constant. Note that there are 2 Mo isotopes, ${ }^{95} \mathrm{Mo}$ and ${ }^{97} \mathrm{Mo}$, that have slightly different gyromagnetic ratios and this leads to a broadening of the ZFNMR spectra. Unfortunately, it is not possible to estimate the magnitude of $A$ and hence it is not possible to estimate the Mo moment from the ZFNMR data. 


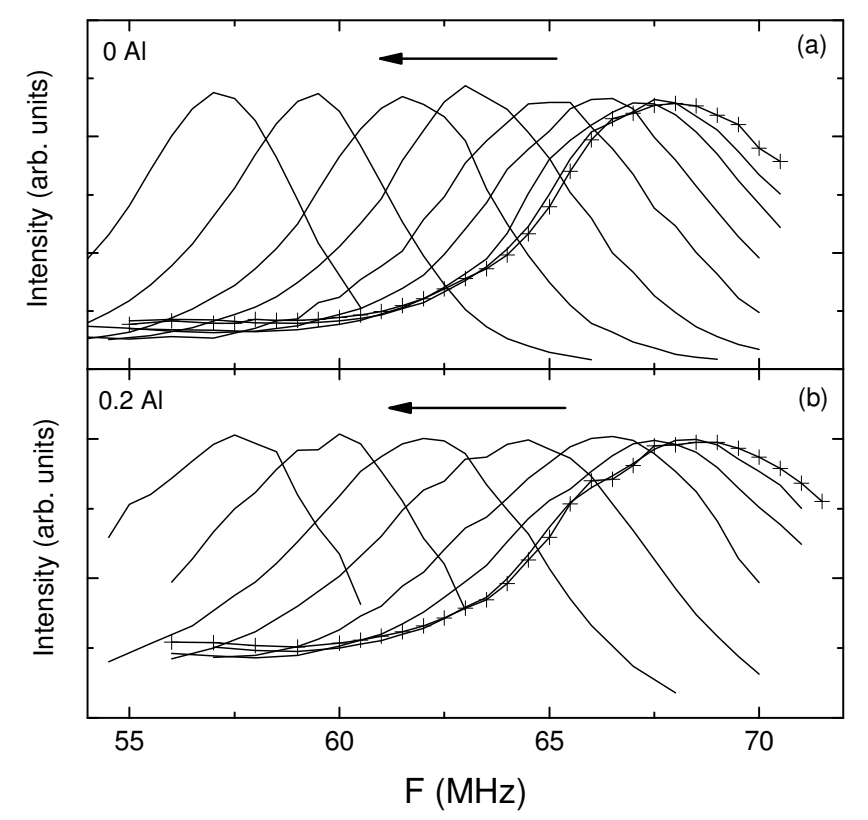

Figure 6.7: Plot of the ZFNMR spectra for $\mathrm{Sr}_{2} \mathrm{Fe}_{1-x} \mathrm{Al}_{x} \mathrm{MoO}_{6}$ with $x=0$ for temperatures of $10 \mathrm{~K}, 25 \mathrm{~K}, 50 \mathrm{~K}, 75 \mathrm{~K}, 100 \mathrm{~K}, 125 \mathrm{~K}, 150 \mathrm{~K}, 175 \mathrm{~K}$ and 200 $\mathrm{K}$ (a) and $x=0.2$ for temperatures of $10 \mathrm{~K}, 25 \mathrm{~K}, 50 \mathrm{~K}, 75 \mathrm{~K}, 100 \mathrm{~K}, 125 \mathrm{~K}$, $150 \mathrm{~K}$ and $175 \mathrm{~K}(\mathrm{~b})$. The arrow indicates increasing temperature. The 10 $\mathrm{K}$ data is indicated by the plus symbols.

It can be seen in figure 6.7 that there is a systematic decrease in $F$ with increasing temperature. The resultant peak frequencies are plotted in figure 6.8. In a 3D Heisenberg model [27], F should decrease as,

$$
F=F_{0}\left(1-a_{0} T^{n}\right)
$$

where the magnitude of $a_{0}$ is determined by the spin-wave dispersion and $n$ is 1.5. This is known as Bloch's law and assumes low temperatures and a spin-wave dispersion of the form, $\epsilon(q)=D q^{2}$. A decrease in the exchange 
energy, $J$, can lead to a decrease in $D$ and $T_{c}$ that will result in an increase in $a_{0}$.

We show in figure 6.8 a fit to equation 6.4 when the data is plotted as $F$ (figure 6.8(a)) and $1-F / F_{0}$ (figure 6.8(b)) using $F_{0}=67.78 \mathrm{MHz}$ for $x=0$ and $F_{0}=68.60 \mathrm{MHz}$ for $x=0.2$. We first note that there is a small increase in $F_{0}$ that could be interpreted in terms of a small increase $(\backsim 1.1 \%)$ in the moment on the Mo site that could be associated with the electronic doping mentioned earlier. It was found that the data can be fitted with $n=1.86$ for $x=0$ and $x=0.2$ and $a_{0}=9.3 \times 10^{-6} \mathrm{~K}^{-1.86}$ for $x=0$ and $a_{0}=12.5 \times 10^{-6} \mathrm{~K}^{-1.86}$ for $x=0.2$. The value of $n$ is higher than that expected in a 3D Heisenberg model and it is also higher than that found when fitting the $6 \mathrm{~T}$ magnetisation data from the pure sample $(n=1.5)$ [67]. It should be noted that Mo ZFNMR only probes the Mo moment while the high-field magnetisation probes the Mo moment and the Fe moment. Thus, the appearance of a different $n$ for both measurement methods indicates that the spin-wave dispersion is complicated in this compound. Any additional analysis will require temperature-dependent neutron diffraction measurements.

There is a small increase in $a_{0}$ that might be expected from the decrease in $T_{c}$. This can be illustrated by noting that in a mean field model a decrease in $T_{c}$ can occur via a drop in $J$. This will affect the spin-wave dispersion and can lead to a decrease in $D$ and hence an increase in $a_{0}$. 

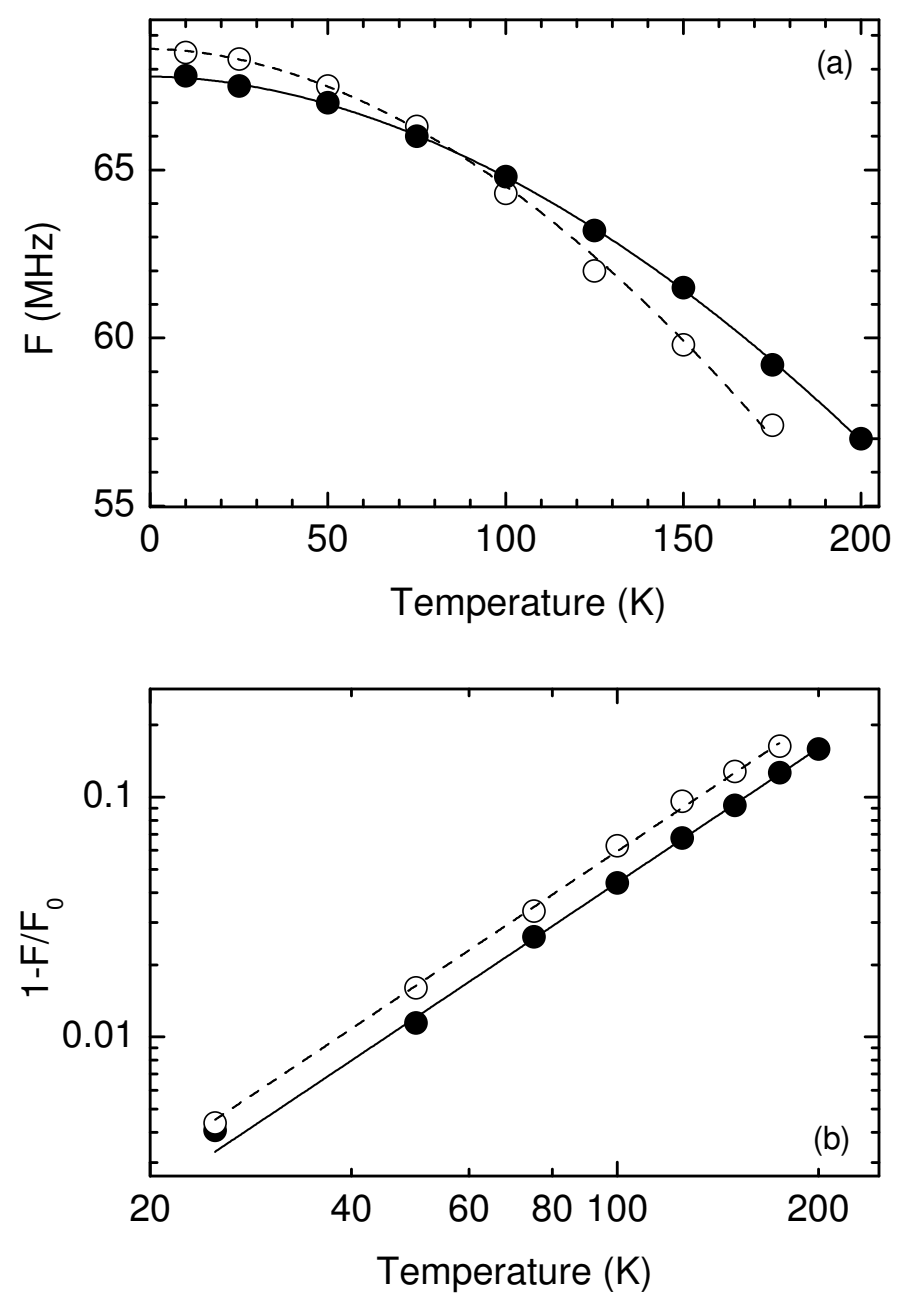

Figure 6.8: (a) Plot of the peak ZFNMR frequency, $F$, against temperature for $\mathrm{Sr}_{2} \mathrm{Fe}_{1-x} \mathrm{Al}_{x} \mathrm{MoO}_{6}$ with $x=0$ (filled circles) and $x=0.2$ (open circles). (b) Plot of the $\log$ of $1 / F-F_{0}$ against the $\log$ of the temperature for $\mathrm{Sr}_{2} \mathrm{Fe}_{1-x} \mathrm{Al}_{x} \mathrm{MoO}_{6}$ with $x=0$ (filled circles) and $x=0.2$ (open circles). The curves are fits to the data using equation 6.4. 


\subsection{Summary}

In summary, it was found that partial substitution of $\mathrm{Fe}$ by $\mathrm{Al}$ in $\mathrm{Sr}_{2} \mathrm{Fe}_{1-x} \mathrm{Al}_{x} \mathrm{MoO}_{6}$ leads to changes in the thermoelectric power that could be interpreted in terms of an electron-doping effect. This can occur if the Fe valence is below $3+$ as reported from a Mössbauer study on the pure compound. The Curie temperature decreases with increasing $x$, which can be attributed to $3 \mathrm{D}$ random site dilution. The saturation moment per f.u., as obtained from magnetisation measurements, is reduced with increasing $x$. However, the Mo moment, as probed by Mo ZFNMR, shows a small increase of $\sim 1.1 \%$ for $x=0.2$. The temperature-dependence of the Mo moment, as probed by Mo ZFNMR, can be fitted to a power law, $1-a_{0} T^{n}$, but $n(1.86)$ is greater than that expected in a 3D Heisenberg model $(n=1.5) . \quad a_{0}$ is found to increase with $x$, which implies a change in the spin-wave dispersion that might be expected from the decrease in $T_{c}$. While there are systematic changes in the thermoelectric power, magnetisation and ZFNMR data with increasing $x$, it was found that there are no consistent changes in the resistivity or MR at $77 \mathrm{~K}$. It is thus clear that the disorder in the grain boundaries varies non-systematically among the samples. 


\section{Chapter 7}

\section{Magnetic and transport properties of oxygen deficient $\mathrm{SrFeO}_{3-\delta}$}

Recently an increase in the resistivity has been reported below $70 \mathrm{~K}$ in $\mathrm{SrFeO}_{3-\delta}$ accompanied by a large magnetoresistance $[40,37,99,100]$. It has been noted that the increase in the resistivity as well as the magnetoresistance are reminiscent of the Verwey transition [40] long known in $\mathrm{Fe}_{3} \mathrm{O}_{4}[101,102]$ arising from a charge-ordered state of the Fe ions in one of the two inequivalent $\mathrm{Fe}$ sites.

The Verwey transition in $\mathrm{Fe}_{3} \mathrm{O}_{4}$ is also accompanied by a large change in the thermoelectric power (TEP) $[43,44]$ that is much larger than the one seen near the metal-insulator transition temperature in the manganites [103]. It is therefore of interest to see if a Verwey-like increase in the resistivity in $\mathrm{SrFeO}_{3-\delta}$ is also accompanied by a large change in the thermoelectric power and ascertain the $\delta$-values where this occurs. It should be noted that there is no temperature dependence of the thermoelectric power available in the literature on $\mathrm{SrFeO}_{3-\delta}$ and very few studies exist where jumps in the TEP have been observed and modelled [103]. In addition we will show that there is a close relationship between the thermoelectric power at room temperature and the oxygen content.

In this chapter we present magnetic and transport measurements per- 
formed on a series of oxygen deficient $\mathrm{SrFeO}_{3-\delta}$ compounds. $\mathrm{Many}_{\mathrm{SrFeO}} \mathrm{C}_{3-\delta}$ samples with various amounts of oxygen content were prepared by solidstate reaction (see chapter 3). As mentioned in chapter 3, thermal gravimetry analysis was not available at this time in order to determine the oxygen content. We show that the magnetic data provide a method to estimate the oxygen deficiency $(\delta)$ in $\mathrm{SrFeO}_{3-\delta}$. Some of the results presented in this chapter have been published in Physical Review B [104].

\subsection{X-ray diffraction of $\mathrm{SrFeO}_{3-\delta}$}

X-ray diffraction measurements were performed on all the samples and show single phase material within the limit of detectibility. Figure 7.1 presents the two end members, $\Delta \delta=0.05$ and 0.28 , and we show in the next section that they are close to $\mathrm{SrFeO}_{2.95}$ and $\mathrm{SrFeO}_{2.72}$ respectively. The XRD patterns clearly demonstrate a shift of the peaks to lower angles as the oxygen deficiency increases. In addition, a splitting of some of the peaks is observed (e.g. the (200) peak) as $\Delta \delta$ increases (see inset of figure 7.1). These features indicate a change in the structure, which has been already observed in $\mathrm{SrFeO}_{3-\delta}$ [41, 100]. It is known that this compound undergoes a change in the structure from cubic to orthorhombic passing through a tetragonal phase. 


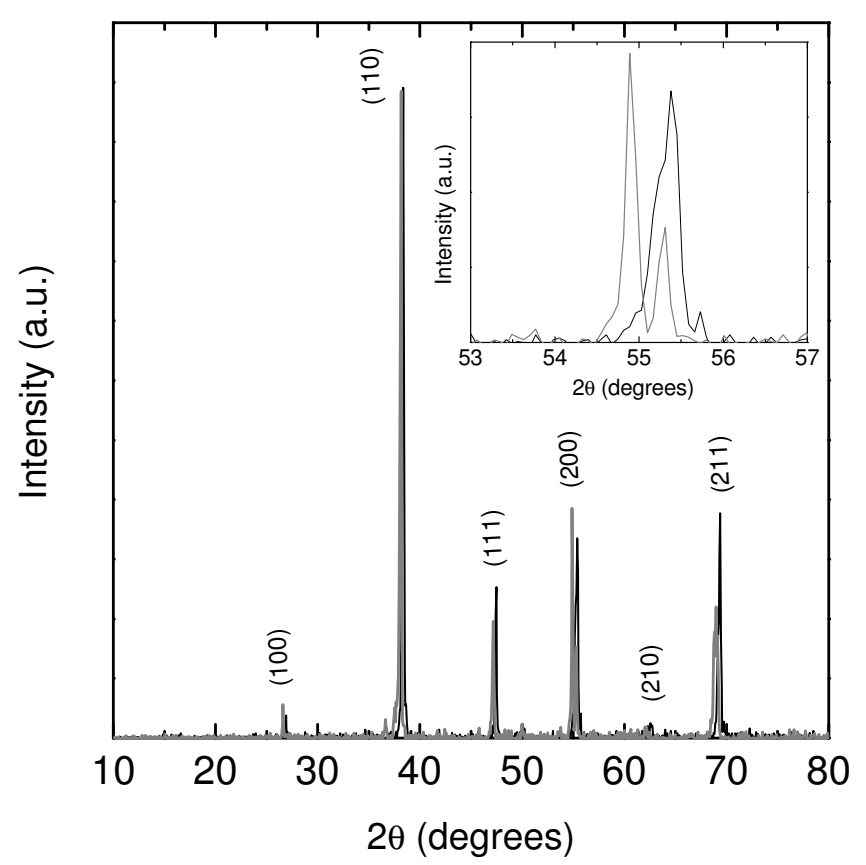

Figure 7.1: XRD patterns of the $\mathrm{SrFeO}_{2.95}$ (black) and $\mathrm{SrFeO}_{2.72}$ (grey) compounds. Inset: zoom of the (200) reflection peak where a splitting of the peak is observed.

\subsection{Magnetic response}

\subsubsection{Determination of $\delta$ from the magnetic measurements}

The Zero Field Cooled (ZFC) and Field Cooled (FC) magnetisation data are plotted in figure 7.2 at $1 \mathrm{~T}$ for three of the samples. Turning first to the asprepared sample $(\Delta \delta=0)$, the peak observed at $\sim 140 \mathrm{~K}$ is associated with helical antiferromagnetic order from $\mathrm{SrFeO}_{3}$ [34]. There is also a shoulder at $\sim 70 \mathrm{~K}$ that arises from antiferromagnetic order in the tetragonal $\mathrm{Sr}_{8} \mathrm{Fe}_{8} \mathrm{O}_{23}$ phase and by comparing these features with reports in the liter- 
ature $[40,37,105]$ we concluded that this sample contains a mixture of cubic and tetragonal phases and that the stoichiometry is close to $\mathrm{SrFeO}_{2.95}$.

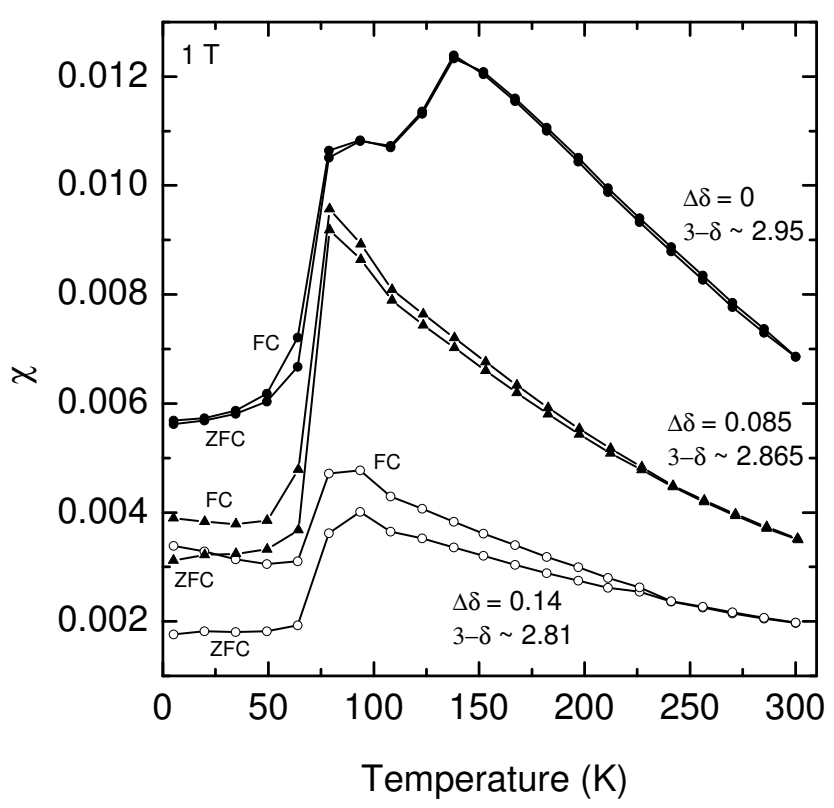

Figure 7.2: Zero Field Cooled (ZFC) and Field Cooled (FC) magnetisation at $1 \mathrm{~T}$ for $\Delta \delta=0\left(\mathrm{SrFeO}_{2.95}\right), \Delta \delta=0.085\left(\mathrm{SrFeO}_{2.865}\right)$ and $\Delta \delta=0.14$ $\left(\mathrm{SrFeO}_{2.81}\right)$.

The $140 \mathrm{~K}$ peak is entirely absent in the $\mathrm{SrFeO}_{2.865}$ sample, replaced by a strong and sharp peak at $\sim 70 \mathrm{~K}$, confirming that it is predominantly the $\mathrm{Sr}_{8} \mathrm{Fe}_{8} \mathrm{O}_{23}$ phase. There is, in addition, a ZFC-FC irreversibility starting at $\sim 240 \mathrm{~K}$, seen also in the $\mathrm{SrFeO}_{2.81}$ sample where the peak at $70 \mathrm{~K}$ is not as pronounced. These results establish the presence of the orthorhombic $\mathrm{Sr}_{4} \mathrm{Fe}_{4} \mathrm{O}_{11}$ phase $[100,39]$ that shows a Néel temperature of $\sim 240 \mathrm{~K}$. It is clear that the magnetic data can provide information on the oxygen 
deficiency in $\mathrm{SrFeO}_{3-\delta}$. Furthermore, additional ZFC and FC measurements were performed in other $\Delta \delta$ samples (not shown here) and confirmed this good correlation between the amplitude of the $T_{N}$ peaks and the stoichiometries. Finally, we will present later in this chapter that the transport measurements are also in good agreement with the given stoichiometries.

\subsubsection{Magnetic measurements}

ZFC and FC magnetisation data at $6 \mathrm{~T}$ are shown in figure 7.3 for the $\mathrm{SrFeO}_{2.95}, \mathrm{SrFeO}_{2.80}$ and $\mathrm{SrFeO}_{2.72}$ samples.

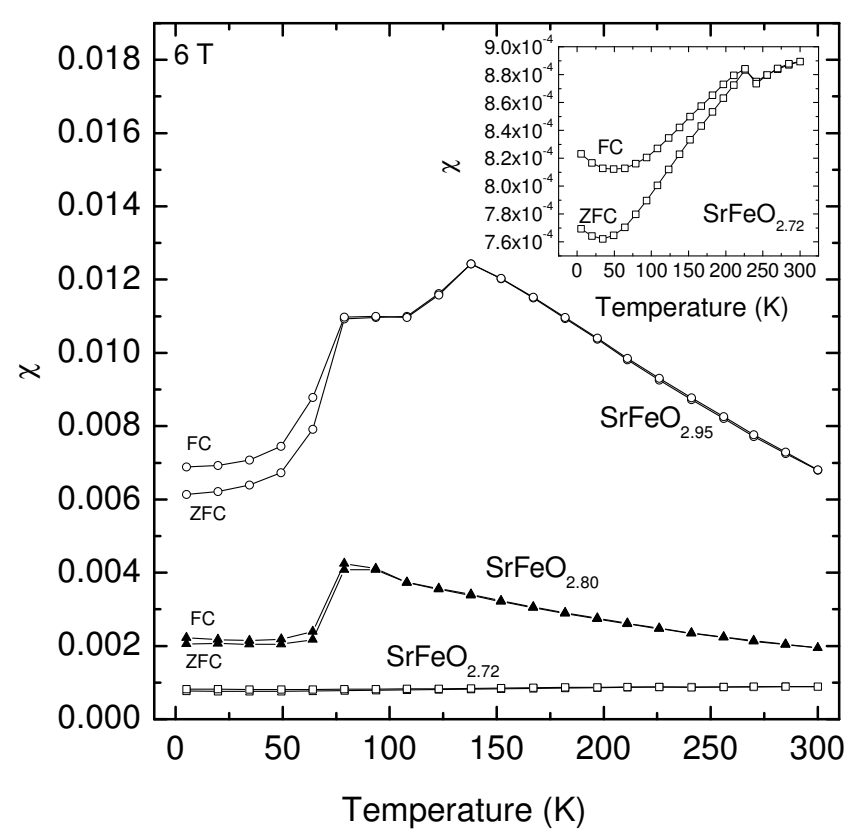

Figure 7.3: Zero Field Cooled (ZFC) and Field Cooled (FC) magnetisation at $6 \mathrm{~T}$ for $\mathrm{SrFeO}_{2.95}, \mathrm{SrFeO}_{2.80}$ and $\mathrm{SrFeO}_{2.72}$. Inset: zoom in of the $\mathrm{SrFeO}_{2.72}$ measurement. 
All the curves are in good agreement with the $1 \mathrm{~T}$ data presented before and therefore show that the determination of the oxygen content using the magnetisation data is not a function of the applied magnetic field. The third sample, $\mathrm{SrFeO}_{2.72}$ has a weak susceptibility and a zoom in is shown in the inset of figure 7.3. It clearly shows irreversibility at $240 \mathrm{~K}$ but without any sign of a peak at $\backsim 70 \mathrm{~K}$, which demonstrates the predominance of the orthorhombic phase. Therefore, all the samples show a departure between the FC and ZFC measurements starting at either $\backsim 70 \mathrm{~K}$ or $\backsim$ $240 \mathrm{~K}$ depending on their antiferromagnetic ordering temperature. These could originate from either a spin-glass behaviour or spin canting. In order to find out more about the origin of the ZFC-FC irreversibility further magnetisation measurements were performed such as ac magnetic susceptibility.

ac magnetic susceptibility $\left(\chi^{\prime}\right)$ measurements were carried out as a function of temperature on the $\mathrm{SrFeO}_{2.80}$ and $\mathrm{SrFeO}_{2.72}$ samples at five different frequencies $(\omega=33,100,303,690$ and $1000 \mathrm{~Hz})$ and are shown in figure 7.4. All the curves lie on top of each other for both samples, which implies that there is no frequency dependence up to $1000 \mathrm{~Hz}$. This is confirmed by Srinath et al. who have not seen any change in $\chi^{\prime}$ up to $10 \mathrm{kHz}$ [100]. This behaviour is not typical of a normal spin-glass system where a $\omega$ dependence is expected [106]. This result seems to be inconsistent with the density-functional calculation performed by Vidya et al. where a spin-glass like behaviour was expected either in $\mathrm{FeO}_{5}$ or distorted $\mathrm{FeO}_{6}$ and where the other phase was a G-type antiferromagnet [107].

In order to further investigate the ZFC-FC irreversibility, we focused our measurements on samples being between the tetragonal $(3-\delta=2.875)$ and orthorhombic $(3-\delta=2.75)$ phases, which contain the distorted $\mathrm{FeO}_{6}$ and five-fold coordinated $\mathrm{FeO}_{5}$ in various proportion (see chapter 2).

FC measurements were carried out at $25 \mathrm{mT}$ on four samples $3-\delta=2.81$, 2.80, 2.76 and 2.75 (see figure 7.5). It is clear that all the compounds have the FC departure at $240 \mathrm{~K}$ which is the mark of the orthorhombic phase. 


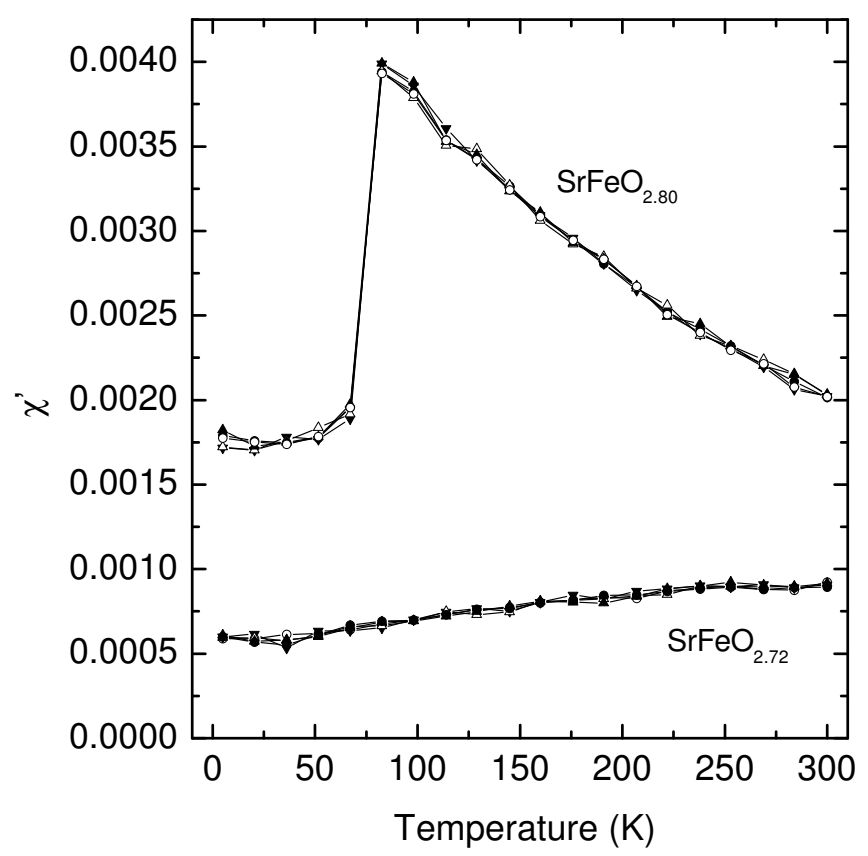

Figure 7.4: Temperature dependence of the ac susceptibility for the $\mathrm{SrFeO}_{2.80}$ and $\mathrm{SrFeO}_{2.72}$ samples at five different frequencies $\omega=33,100$, 303, 690 and $1000 \mathrm{~Hz}$.

The susceptibility at $5 \mathrm{~K}$ increases versus the oxygen deficiency and is plotted in the inset of figure 7.5. This increase could originate from the distorted $\mathrm{FeO}_{6}$ octahedra structure where spin canting could be present and therefore leading to a weak ferromagnetic component. This can occur via a Dzyaloshinsky-Moriya (DM) interaction [108, 109]. As the oxygen deficiency increases, the volume fraction of the orthorhombic phases increases and therefore the ferromagnetic component increases. 


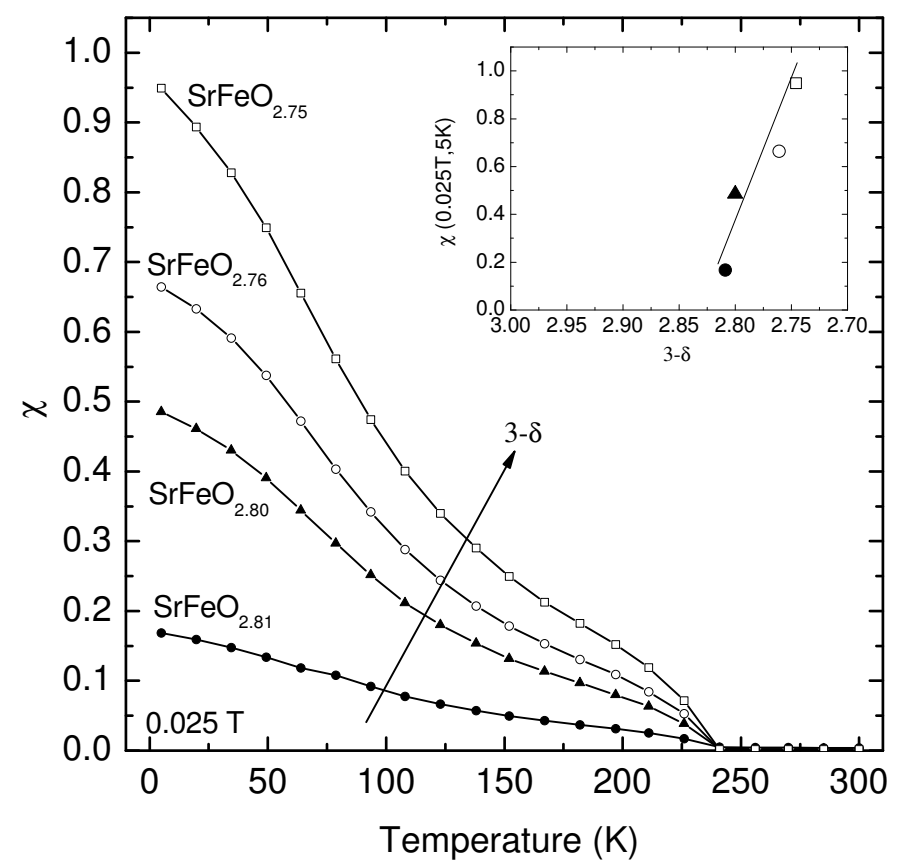

Figure 7.5: FC measurements at $25 \mathrm{mT}$ on samples having an oxygen deficiency of 3- $\delta=2.81$ (filled circle), 2.80 (filled triangle), 2.76 (open circle) and 2.75 (open square). Inset: $\chi$ at $5 \mathrm{~K}$ versus $3-\delta$. The solid line is a guide for the eye.

\subsection{Temperature dependence of the resistivity}

\subsubsection{Zero field resistivity measurements}

The resistivity data are plotted in figure 7.6 for the (a) $\mathrm{SrFeO}_{2.95}$, (b) $\mathrm{SrFeO}_{2.865}$ and (c) $\mathrm{SrFeO}_{2.81}$ samples. All the samples show a large increase in the resistivity at $\backsim 70 \mathrm{~K}$ of up to an order of magnitude associated with chargeordering in the tetragonal phase $[40,37,99,42]$. It is important to note that our polycrystalline samples possess inter-grain and intra-grain contribu- 


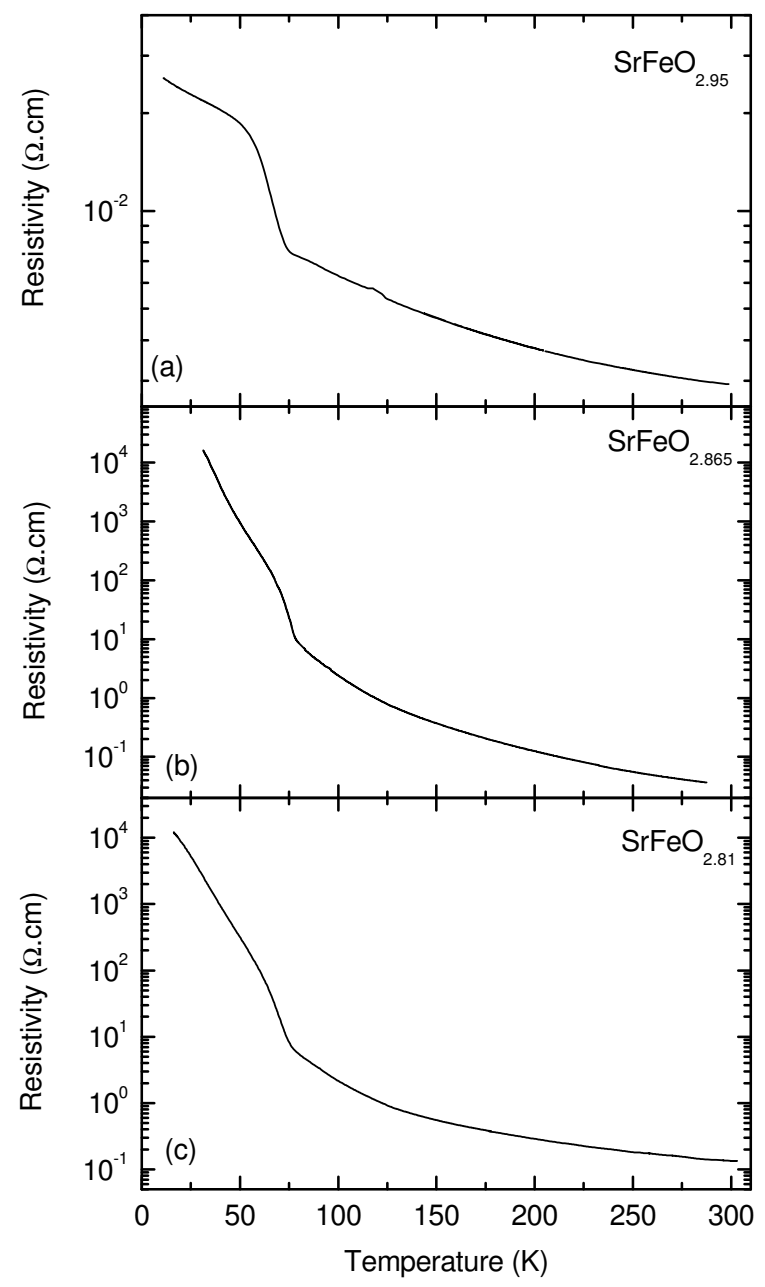

Figure 7.6: Temperature dependence of the resistivity for (a) $\mathrm{SrFeO}_{2.95}$, (b) $\mathrm{SrFeO}_{2.865}$ and (c) $\mathrm{SrFeO}_{2.81}$.

tions to the resistivity. The feature at $70 \mathrm{~K}$ originates from the intra-grain contribution while the general trend, the increase in the resistivity when the temperature decreases, is probably due to the inter-grain contribution. For this latter reason, the $\mathrm{SrFeO}_{2.95}$ sample does not show the metallic- 
like resistivity observed in single crystals above $\backsim 70 \mathrm{~K}$ [37]. The resistivity increases with increasing oxygen deficiency as reported by other studies $[37,100]$ arising from mixed-phase conduction involving an increasing fraction of semiconducting $\mathrm{Sr}_{4} \mathrm{Fe}_{4} \mathrm{O}_{11}$.

\subsubsection{Magneto-transport behaviour of $\mathrm{SrFeO}_{3-\delta}$}

The temperature dependence of the resistivity at zero field (grey curve) and $12 \mathrm{~T}$ (black curve) is plotted in figure 7.7 for the (a) $\mathrm{SrFeO}_{2.95}$, (b) $\mathrm{SrFeO}_{2.80}$ and (c) $\mathrm{SrFeO}_{2.72}$ samples.

Figures 7.7(a) and (b) present a large increase in the resistivity at $\backsim$ $70 \mathrm{~K}$, which has been associated with charge-ordering in the tetragonal phase as mentioned before. It means that these two samples contain some of the $\mathrm{Sr}_{8} \mathrm{Fe}_{8} \mathrm{O}_{23}$ phase, which is in perfect agreement with our given stoichiometries. In addition, at low temperature, $\mathrm{SrFeO}_{2.95}$ has a six orders of magnitude smaller resistivity than $\mathrm{SrFeO}_{2.80}$.

It can be seen in figures 7.7(a) and (b) that under an applied magnetic field of $12 \mathrm{~T}$, a hysterisis is present at the charge-ordering temperature. The origin of the hysterisis has not been clearly explained and was surprisingly observed by other investigators at zero field [37]. It is important to mention that our zero field measurements were performed in a dewar and not in the same cryostat where the $12 \mathrm{~T}$ measurements were performed. It is known that a small remanent field could be still present when a magnet is turned off and could have a possible effect on the zero field measurements. Furthermore, we have carried out the zero field measurements before the $12 \mathrm{~T}$ measurements to avoid any magnetic history behaviour. This could be another possible explanation for the hysterisis signal observed under zero field by the other investigators. Further work would be required to ascertain the origin of this hysterisis. 


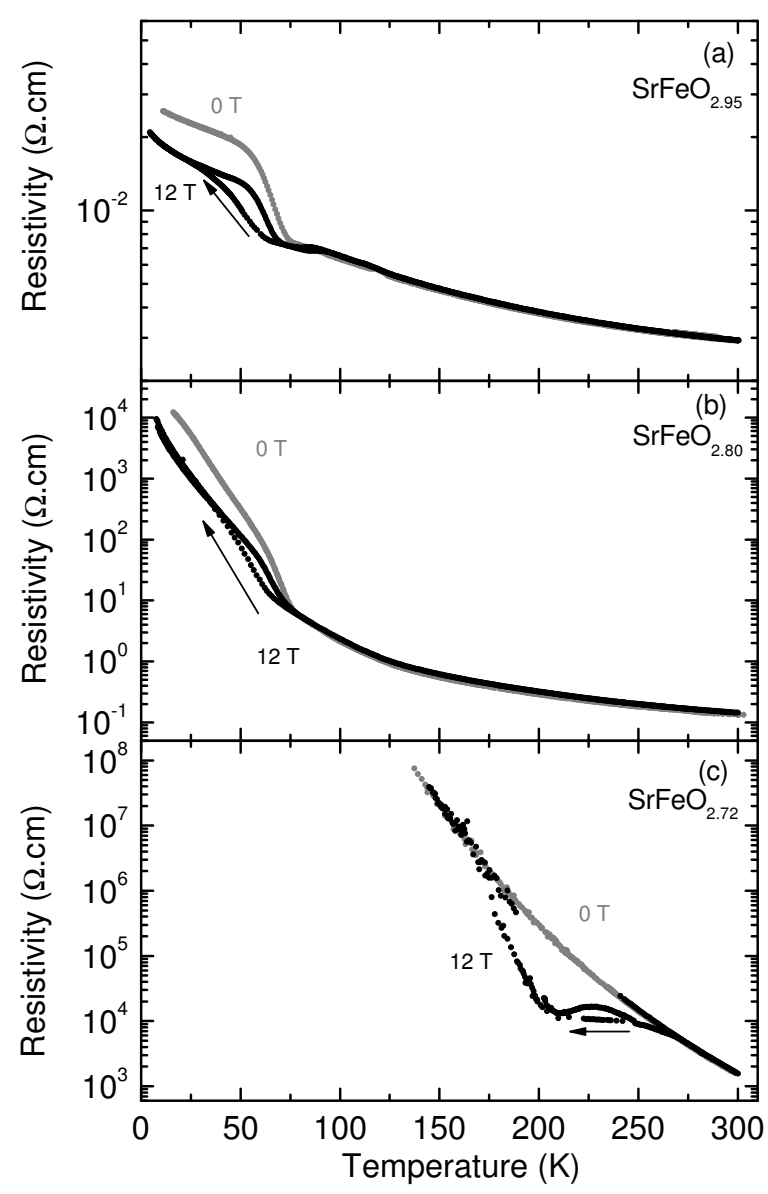

Figure 7.7: Temperature dependence of the resistivity at zero field (grey curve) and $12 \mathrm{~T}$ (black curve) for (a) $\mathrm{SrFeO}_{2.95}$, (b) $\mathrm{SrFeO}_{2.80}$ and (c) $\mathrm{SrFeO}_{2.72}$.

Moving now to figure 7.7(c), it can be seen that the sample shows a semiconductor type behaviour at zero field reaching values up to $10^{8} \Omega . \mathrm{cm}$ at around $130 \mathrm{~K}$. The measurement could not be performed at lower temperature as it was affected by the input impedance of the nanovoltmeter. It was shown from a band structure calculation that semiconductor be- 
haviour should be expected for $\mathrm{SrFeO}_{2.75}$ [107].

Under an applied magnetic field of $12 \mathrm{~T}$, a more complex feature is observed in the $\mathrm{SrFeO}_{2.72}$ compound in comparison to the other samples. First there is a hysterisis at around $240 \mathrm{~K}$, which is perfectly in agreement with the transition temperature of the orthorhombic phase measured in the magnetic data (figure 7.3). In addition, on the top of this hysterisis signal, a major deviation to lower resistivity values from the zero field data is observed, which switched back onto the zero field data at lower temperature $(\backsim 175 \mathrm{~K})$. Due to the unexpected nature of this signal, this measurement was performed a second time on this sample and on another sample having a similar oxygen deficiency $(3-\delta=2.75)$. Identical signals were recorded and therefore certify the existence of this magnetoresistance behaviour in the samples containing only the orthorhombic phase. On the other hand, this behaviour was not observed in $\mathrm{SrFeO}_{2.80}$, which contains the tetragonal and orthorhombic phases. This suggests that the tetragonal phase short circuits this magnetoresistance behaviour and is observed for compounds containing only the orthorhombic phase. Even in the presence of a mixture of the two phases, the tetragonal phase could be the preferential pathway and therefore the magnetoresistance behaviour from the orthorhombic phases would not be observed in the resistivity data. At this stage, this behaviour is not well-understood and further work would be required to establish its origin. However, these results are very interesting as for a first time magnetoresistance close to room temperature was observed in the $\mathrm{SrFeO}_{3-\delta}$ compound. Note that the magnetoresistance is also much larger than the one observed in the double perovskite (chapter 4). These results provide a clear direction for future work. 


\subsection{Thermoelectric power measurements}

\subsubsection{Anomalous temperature dependence of the thermo- electric power}

Thermoelectric power data for $3-\delta=2.95,2.865,2.81,2.79$ and 2.78 were measured as a function of temperature and the results are shown in figure 7.8. An abrupt drop in the TEP can be observed for the $\mathrm{SrFeO}_{2.865}$ sample (figure $7.8(\mathrm{~b})$ ) at $\sim 70 \mathrm{~K}$ where the drop in the TEP is $\Delta S \backsim 118 \mu \mathrm{V} / \mathrm{K}$. This decrease is clearly associated with the $\mathrm{Sr}_{8} \mathrm{Fe}_{8} \mathrm{O}_{23}$ phase, which is dominant in that sample. An abrupt fall in the TEP is observed for higher oxygen deficiencies, as expected for a mixed-phase sample, with a magnitude that decreases with $\delta$ and nearly disappears for $3-\delta=2.78$. Thus, the magnitude of the effect decreases as the $\mathrm{Sr}_{8} \mathrm{Fe}_{8} \mathrm{O}_{23}$ fraction decreases, although it disappears sooner than the limit 3- $\delta=2.75$ where $\mathrm{Sr}_{4} \mathrm{Fe}_{4} \mathrm{O}_{11}$ is expected to be the only remaining phase. The drop occurs at the magnetic ordering temperature and the resistivity change found in the $\mathrm{SrFeO}_{2.865}$ sample; the effect is clearly related to charge-ordering in the $\mathrm{Sr}_{8} \mathrm{Fe}_{8} \mathrm{O}_{23}$ fraction of the sample. The large drop in the TEP below $\backsim 70 \mathrm{~K}$ signals a change in the nature of the charge carriers that may be expected to follow from chargeordering.

A large decrease in the TEP was observed when the charge-ordering is associated with a Verwey-like transition. For example, a drop of more than $70 \mu \mathrm{V} / \mathrm{K}$ was reported from TEP measurements on $\mathrm{Fe}_{3} \mathrm{O}_{4}$ near the Verwey transition temperature where charge-ordering is not accompanied by a metal-insulator transition and the resistivity increases by approximately two orders of magnitude $[43,44]$.

This can be compared with $\mathrm{La}_{0.67} \mathrm{Ca}_{0.33} \mathrm{MnO}_{3}$ where a drop of $\sim 6 \mu \mathrm{V} / \mathrm{K}$ was observed near the metal-insulator transition temperature [103]. Thus, the large drop in the TEP observed for the $\mathrm{Sr}_{8} \mathrm{Fe}_{8} \mathrm{O}_{23}$ phase as well as the small increase in the resistivity ( $\sim 1$ order of magnitude) would appear to 


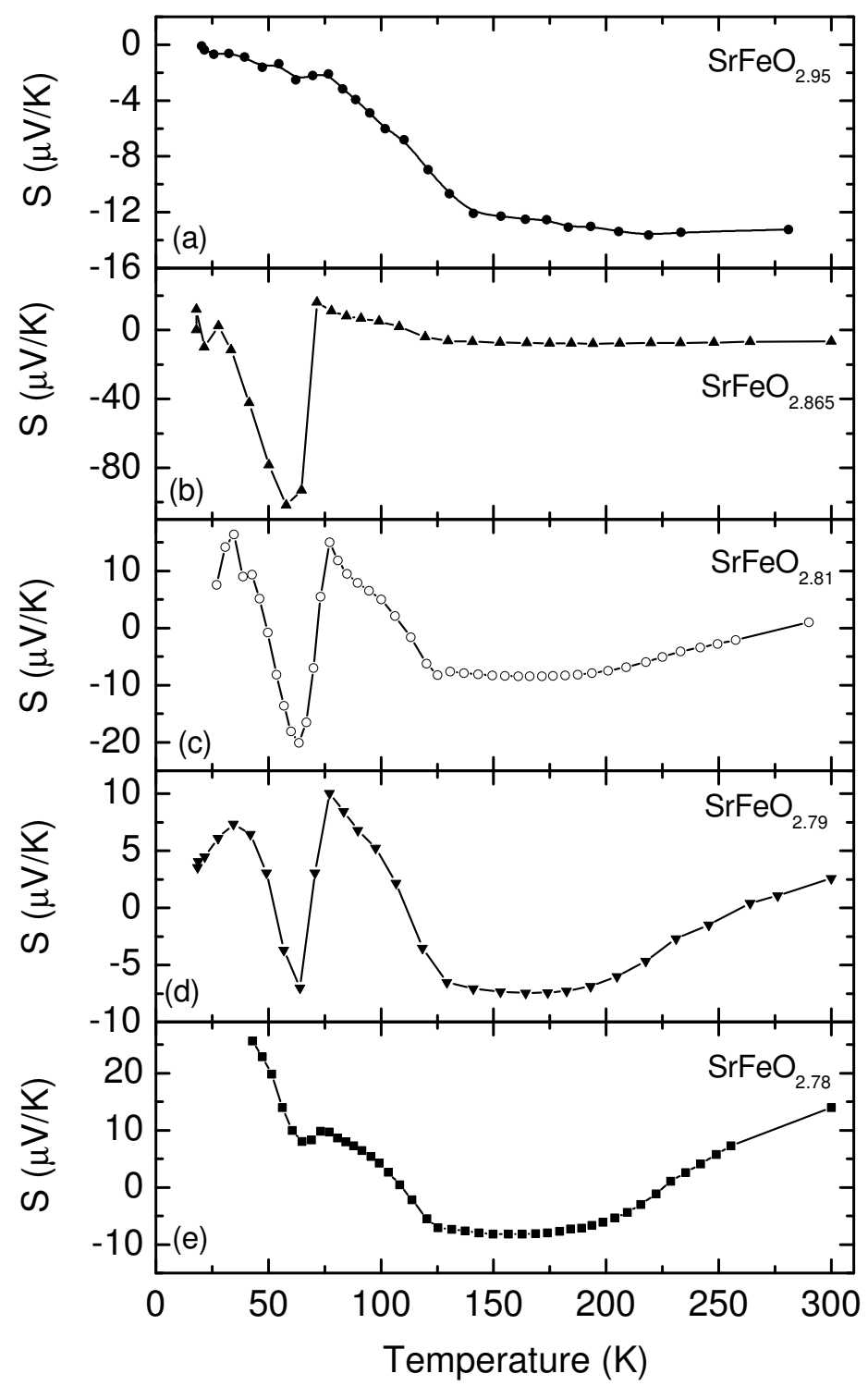

Figure 7.8: TEP versus temperature for (a) $\mathrm{SrFeO}_{2.95}$, (b) $\mathrm{SrFeO}_{2.865}$, (c) $\mathrm{SrFeO}_{2.81}$, (d) $\mathrm{SrFeO}_{2.79}$ and (e) $\mathrm{SrFeO}_{2.78}$. 
be reminiscent of a Verwey-like transition occurring at the magnetic ordering temperature in $\mathrm{Sr}_{8} \mathrm{Fe}_{8} \mathrm{O}_{23}$.

We show below that the composition dependence of the large drop in the TEP in our data can be described in terms of charge-ordering of the $\mathrm{Sr}_{8} \mathrm{Fe}_{8} \mathrm{O}_{23}$ phase within an inhomogeneous two-phase model similar to that used for $\mathrm{La}_{0.67} \mathrm{Ca}_{0.33} \mathrm{MnO}_{3}$ [103]. We based our model on the effectivemedium approach, in which the transport coefficient of a composite is represented by a weighted average of the two components, their volume fractions and geometric arrangements. Therefore, by using equation 6 from Jaime et al. we can rewrite the TEP under the following form [103],

$$
\begin{aligned}
S(x) & =S_{2}\left(\frac{\rho_{1}}{\rho_{1}-\rho_{2}}-\frac{\kappa(x) \rho(x)}{\kappa_{1} \rho_{1}-\kappa_{2} \rho_{2}}-\frac{\rho_{1} \rho_{2}\left(\kappa_{2}-\kappa_{1}\right)}{\left(\kappa_{1} \rho_{1}-\kappa_{2} \rho_{2}\right)\left(\rho_{1}-\rho_{2}\right)}\right) \\
& +S_{1}\left(\frac{\kappa(x) \rho(x)}{\kappa_{1} \rho_{1}-\kappa_{2} \rho_{2}}-\frac{\rho_{2}}{\rho_{1}-\rho_{2}}-\frac{\rho_{1} \rho_{2}\left(\kappa_{2}-\kappa_{1}\right)}{\left(\kappa_{1} \rho_{1}-\kappa_{2} \rho_{2}\right)\left(\rho_{1}-\rho_{2}\right)}\right)
\end{aligned}
$$

where $\rho_{1}, \rho_{2}$ and $\rho(x), \kappa_{1}, \kappa_{2}$ and $\kappa(x)$ are the resistivity and thermal conductivities of the various phases and the effective medium respectively. Thermal conductivity experiments would be required in single crystals in order to measure this quantity but no single crystal sample growth and instrument was available at this time. However, it is known that the thermal conductivity in polycrystalline compounds change much less than the electrical resistivity and therefore, as assumed by Jaime et al., $\kappa_{1} \approx \kappa_{2} \approx$ $\kappa(x)$ and equation 7.1 can be reduced to,

$$
S(x) \approx S_{2}\left(\frac{\rho_{1}-\rho(x)}{\rho_{1}-\rho_{2}}\right)+S_{1}\left(\frac{\rho(x)-\rho_{2}}{\rho_{1}-\rho_{2}}\right)
$$

if $y=\left(\rho_{1}-\rho(x) /\left(\rho_{1}-\rho_{2}\right), S_{2}=S_{T}\right.$ and $S_{1}=S_{O}$ then we can write that,

$$
S(x)=y S_{T}+(1-y) S_{O}
$$


where $S_{T}$ and $S_{O}$ are the TEPs of the tetragonal and orthorhombic phases respectively $[103,110]$. The fraction $y$ depends upon the electrical resistivities, their volume fractions and geometric arrangement. Note that it was assumed that $\kappa_{1} \approx \kappa_{2} \approx \kappa(x)$ to simplify the equations but one could leave the thermal conductivities in and still use equation 7.3 where $y$ would also have a dependence on $\kappa_{1}, \kappa_{2}$ and $\kappa(x)$.

The TEP from the tetragonal phase was modelled by using the results from the sample with $3-\delta=2.865$ (figure $7.8(\mathrm{~b})$ ), which is close to the $3-\delta$ $=2.875$ expected for that phase. The step at the charge-ordering temperature, $T_{C O}(69 \mathrm{~K})$, requires different fits above and below that temperature. The low temperature data was fitted to,

$$
S_{T}=149 \tanh (T / 26)-4.2 T, T<T_{C O}=69 K
$$

where $S_{T}$ is in units of $\mu \mathrm{V} / \mathrm{K}$. A similar function was used to fit the TEP from some other transition metal oxides $[67,70]$. The first term provides a positive drag-like TEP that vanishes at $T=0$. The second term is linear in temperature characteristic of the diffusion TEP. However, it is too large for a simple metal interpretation, but typical of the diffusion TEP found in the presence of charge hopping [111].

It can be seen in figure 7.9 that the TEP from all of the tetragonalorthorhombic composites track closely at temperatures above $T_{C O}$. Careful comparisons show negligible differences in the temperature range from 70 to $130 \mathrm{~K}$, indicating that in this temperature range the two phases show the same TEP. Thus, by subtracting the fraction of equation 7.4 to the TEP data using equation 7.3 we recovered the orthorhombic-phase TEP,

$$
S_{O}=(4443 / T-41.37)(\tanh (T / 39.1))^{3.256}, 0<T<130 K
$$

and that serves also as $S_{T}$ above $T_{C O}$. 

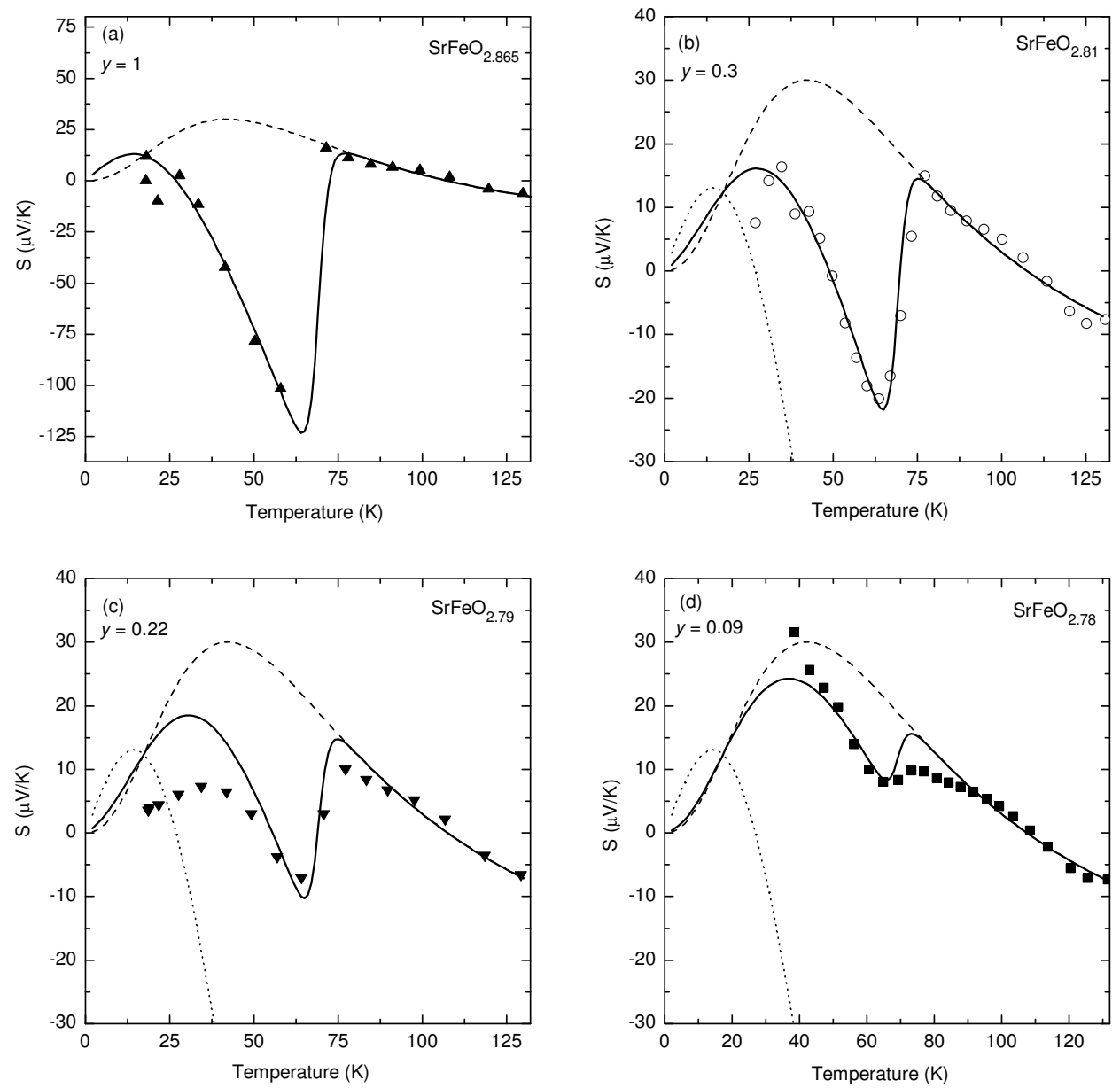

Figure 7.9: TEP versus temperature for (a) $\mathrm{SrFeO}_{2.865}$, (b) $\mathrm{SrFeO}_{2.81}$, (c) $\mathrm{SrFeO}_{2.79}$ and (d) $\mathrm{SrFeO}_{2.78}$. The solid curve is the two phase fit to the data with $y=1,0.3,0.22$ and 0.09 times the tetragonal TEP for (a) $\mathrm{SrFeO}_{2.865}$, (b) $\mathrm{SrFeO}_{2.81}$, (c) $\mathrm{SrFeO}_{2.79}$ and (d) $\mathrm{SrFeO}_{2.78}$ respectively. The orthorhombic TEP is the dashed curve (equation 7.5) and the dotted curve shows the low-temperature contribution of the tetragonal phase (equation 7.4). 
The transition between the low- and high-temperature expressions for $S_{T}$ can be represented by a broadened Heaviside function, $H_{B}=0.5(1-$ $\tanh ((T / 69) / 3))$ and $1-H_{B}$. Therefore by combining equation 7.4 and 7.5 and the Heaviside function, we can re-write $S_{T}$ from 0 to $130 \mathrm{~K}$,

$$
\begin{array}{r}
S_{T}=[149 \tanh (T / 26)-4.2 T][0.5(1-\tanh ((T / 69) / 3))] \\
+\left[(4443 / T-41.37)(\tanh (T / 39.1))^{3.256}\right] \\
{[0.5(1+\tanh ((T / 69) / 3))]} \\
0<T<130 K
\end{array}
$$

Therefore by substituting $S_{T}$ (equation 7.6) and $S_{O}$ (equation 7.5) in equation 7.3 it can be seen that this simple model provides a reasonable fit for all the oxygen concentration (see solid caurve in figure 7.9). We obtained $y$ $=1,0.3,0.22$ and 0.09 for (a) $\mathrm{SrFeO}_{2.865}$, (b) $\mathrm{SrFeO}_{2.81}$, (c) $\mathrm{SrFeO}_{2.79}$ and (d) $\mathrm{SrFeO}_{2.78}$ respectively. A discrepancy between the obtained $y$ values and the oxygen concentration was found. For example by looking at the $3-\delta=$ 2.81 sample it was found that the best fit is for $y=0.3$. However, from the oxygen content, it should contain 0.5 of the tetragonal phase but as mentioned before the $y$ parameter depends upon the electrical resistivities, the thermal conductivities, the volume fractions and geometric arrangement of the two phases.

It can be seen in figure 7.10 that there is an approximately linear decrease in the amplitude of the TEP drop $(\Delta S)$ near $70 \mathrm{~K}$ with increasing oxygen deficiency. It is important to note that the contribution from the tetragonal phase disappears before $3-\delta=2.75$. Sharp percolation effects familiar from resistance measurements of composite systems are not found in the TEP and it is relatively insensitive to the presence of electrically resistive grain boundaries. Therefore, the disappearance of the tetragonal phase before $3-\delta=2.75$ is unlikely to be related to percolative behaviour but almost certainly signals the absence of the tetragonal phase for 3- $\delta$ $<2.78$. Therefore, it is likely that the compounds between 2.75 and 2.78 are actually in the orthorhombic phase but contain some interstitial excess 
oxygen.

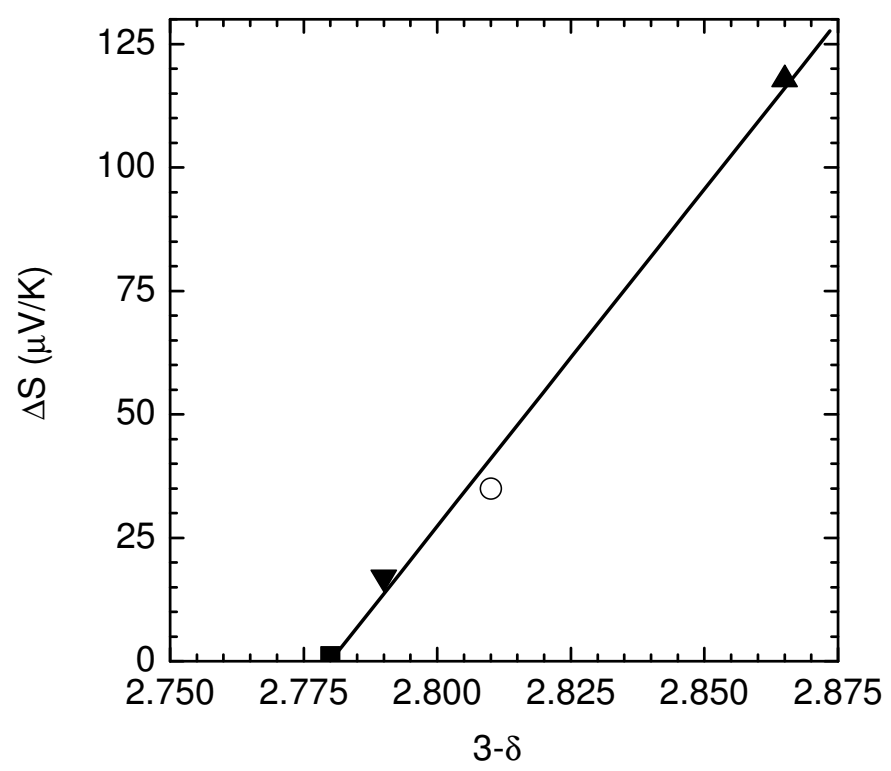

Figure 7.10: Plot of the drop in the TEP, $\Delta S$, against 3- $\delta$ where the up filled triangle is $\mathrm{SrFeO}_{2.865}$, the open circle is $\mathrm{SrFeO}_{2.81}$, the down filled triangle is $\mathrm{SrFeO}_{2.79}$ and the filled square is $\mathrm{SrFeO}_{2.78}$.

\subsubsection{Room temperature thermoelectric power}

The thermoelectric power at room temperature $(S(290 K))$ was measured for various oxygen deficient samples. $S(290 K)$ versus $\delta$ was obtained and converted to $S(290 K)$ versus $R=$ (fraction of $\mathrm{Fe}^{3+}$ )/(total of Fe ions) as presented by the filled circles in figure 7.11 .

It can be seen from this figure that the thermoelectric power increases gently from -13 to $0 \mu \mathrm{V} / \mathrm{K}$ and then rises sharply at $R=0.45$. Then, a drastic transition is observed at $3-\delta=2.75$ going from $\sim 100$ to $-250 \mu \mathrm{V} / \mathrm{K}$ for a 


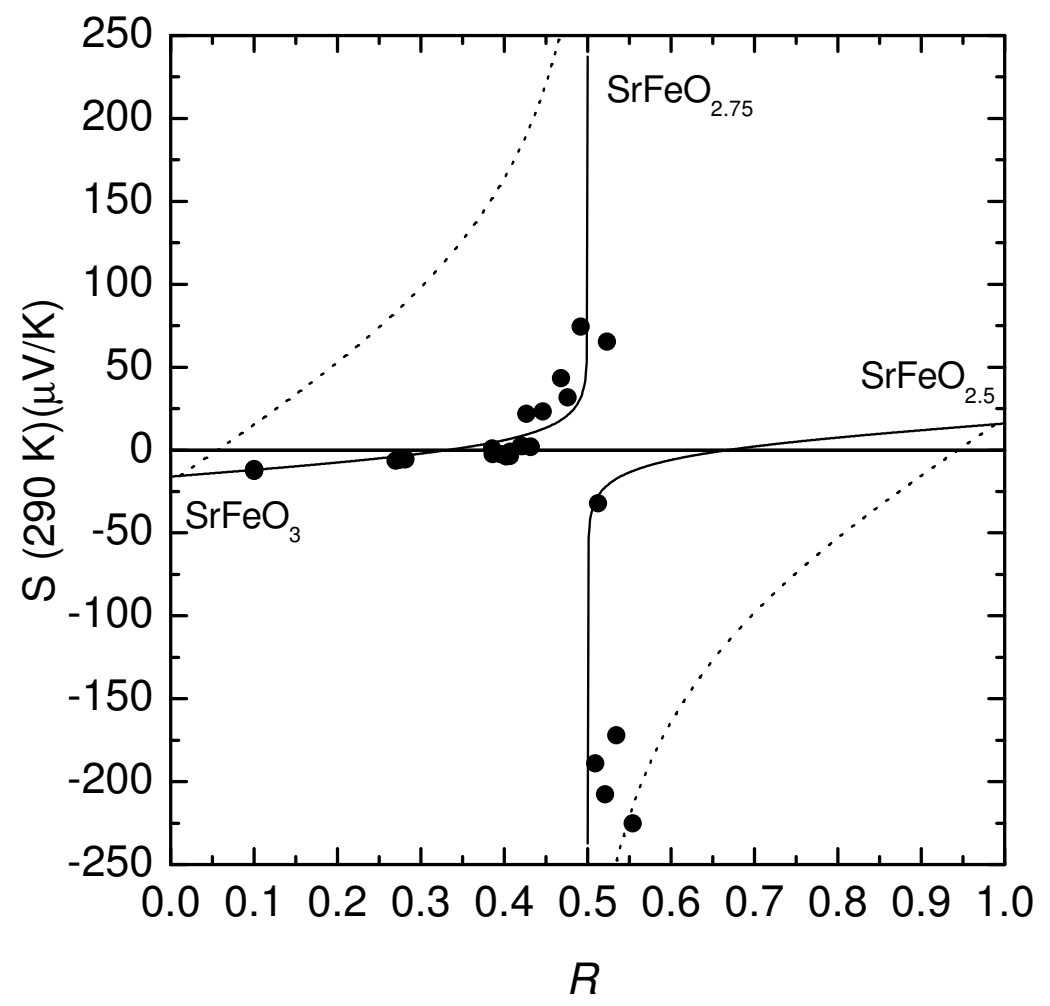

Figure 7.11: Thermoelectric power at room temperature, $S(290 K)$, versus $R=$ (fraction of $\left.\mathrm{Fe}^{3+}\right) /($ total of Fe ions). The dotted line is the Heikes formula (equation 7.7) and the solid line is the best fit (equation 7.8).

small change in $\delta$. It is interesting to see that this transition corresponds to the $50 \% \mathrm{Fe}^{4+}-50 \% \mathrm{Fe}^{3+}$ iron ratio. A similar behaviour was observed in other strongly correlated electron system such as the cobalt oxides [112], for example in $\mathrm{La}_{1-x} \mathrm{Sr}_{x} \mathrm{CoO}_{3}[113,114]$ or $\mathrm{GdBaCo}_{2} \mathrm{O}_{5+x}$ [115]. It has been clearly shown that the ratio of the ions are crucial to enhance the thermoelectric power $[112,113,114]$. This is in good agreement with our sharp transition at exactly $50 \% \mathrm{Fe}^{4+}-50 \% \mathrm{Fe}^{3+}$ iron ratio. This particular ra- 
tio is reinforced by the measurements performed by Poulsen et al. on two $\mathrm{SrFeO}_{3-\delta}$ samples who observed a change in the TEP sign in $\mathrm{SrFeO}_{2.85}$ and $\mathrm{SrFeO}_{2.65}$ and were expecting this change to occur at $\mathrm{SrFeO}_{2.75}$ [116]. The sharp transition observed in the cobalt oxides has been analysed in term of the Heikes formula given by,

$$
S=-\left(\frac{k_{B}}{e}\right) \ln \left(\frac{1-R}{R}\right)
$$

where $k_{B}$ is the Boltzmann constant, $e$ the charge of the electron and $R$ is the fraction of $\mathrm{Fe}^{3+}$ to the total of Fe ions. Equation 7.7 is valid in a narrowband Hubbard model and with a sizable on-site Coulomb interaction in a one dimensional system [117].

However, as can be seen by the dotted line in figure 7.11, the Heikes formula gives a poor representation of our experimental data. This discrepancy could be explained, for example, by the fact that our compounds are inhomogeneous and possess a mixture of phases as proved by our magnetic and transport measurements. Furthermore, this formula is known to be applicable at very high temperature where the TEP is independent of the temperature. Nevertheless, we can still obtain the best fit to our data (solid line in figure 7.11) by using the Heikes formula (equation 7.7) with additional empirical terms and this can be written as,

$$
S(290 K)=-\left(\frac{k_{B}}{10 * e}\right) \ln \left(\frac{1-R}{R}\right)-16
$$

Another possible reason for the sharp transition at $\mathrm{SrFeO}_{2.75}$ could be explained in terms of a change in the conduction carrier types. It was shown from band structure calculation that a gap in the DOS at the Fermi energy is present for the $\mathrm{SrFeO}_{2.75}$ compound [107]. Therefore, as 3- $\delta$ increases in $\mathrm{SrFeO}_{3-\delta}$, the $\mathrm{Fe}^{4+}$ concentration would increase and the carriers would be hole-like and this will provide a positive thermoelectric power. On the other hand, as $3-\delta$ decreases the amount of $\mathrm{Fe}^{3+}$ increases and the system becomes electron-doped and therefore gives a negative TEP re- 
sponse. Further experimental work and analysis would be necessary to ascertain the origin of this behaviour.

\subsection{Summary}

In summary, electronic and magnetic measurements were performed on a series of oxygen deficient $\mathrm{SrFeO}_{3-\delta}$ samples. From the magnetic measurements, it was demonstrated that spin canting could be present in the distorted $\mathrm{FeO}_{6}$ octahedra giving rise to a ferromagnetic component. This can occur via a Dzyaloshinsky-Moriya interaction. Thanks to the temperature dependence of the resistivity at $12 \mathrm{~T}$, we observed that samples containing the orthorhombic phase exhibit a large magnetoresistance at its antiferromagnetic ordering temperature ( $\sim 240 \mathrm{~K})$. The origin of this unusual behaviour is still unclear but this provides clear directions for future work. Regarding the transport measurements, a large drop in the TEP was observed in $\mathrm{SrFeO}_{3-\delta}$ for $3-\delta=2.865$ to 2.78 at the temperature where the $\mathrm{Sr}_{8} \mathrm{Fe}_{8} \mathrm{O}_{23}$ phase magnetically orders and displays charge-ordering. The drop in the TEP data was attributed to a Verwey-like charge-ordering from the $\mathrm{Sr}_{8} \mathrm{Fe}_{8} \mathrm{O}_{23}$ fraction where $\mathrm{SrFeO}_{3-\delta}$ is an inhomogeneous mixture of $\mathrm{SrFeO}_{3}, \mathrm{Sr}_{8} \mathrm{Fe}_{8} \mathrm{O}_{23}$ and $\mathrm{Sr}_{4} \mathrm{Fe}_{4} \mathrm{O}_{11}$. Finally, it was shown that the thermoelectric power at room temperature could be used to determine the oxygen content in $\mathrm{SrFeO}_{3-\delta}$ and where a sharp transition is observed at $\mathrm{SrFeO}_{2.75}$. This latter transition could be explained in terms of the Heikes formula but it is more likely to originate from a two band semiconductor model. 


\section{Summary and conclusions}

In this thesis, we reported new magnetic and transport results on the $\mathrm{Sr}_{2} \mathrm{FeMoO}_{6}$ double perovskite and $\mathrm{SrFeO}_{3-\delta}$ compounds. Chemical substitutions on the various atomic sites and oxygen deficient samples were studied to probe and further understand the unusual properties of these two strongly correlated electron systems. The main conclusions and outlook for future work are summarized here.

In chapter 2, a literature review of the two perovskite systems is presented. We first summarized the properties of the half-metallic and ferromagnetic double perovskites and pointed out some of the remaining questions. It is clear that the recent observation of a tunneling magnetoresistance at room temperature by Kobayashi et al. has triggered major interest in this family of compounds. The magnetoresistance together with the Curie temperature, usually above room temperature, make this compound a promising candidate for spintronics applications.

Then we moved to the oxygen deficient $\mathrm{Fe}$-based perovskite, $\mathrm{SrFeO}_{3-\delta}$, in which much less research has been reported in comparison to the double perovskites. $\mathrm{SrFeO}_{3-\delta}$ presents a wide variety of magnetic transition temperatures, magnetoresistances and resistivities with different oxygen deficiencies. However, the underlying physics is not clear.

Chapter 3 contains the sample preparation details for the two perovskite compounds and also includes the description of the instruments used for 
the characterisation of these compounds.

In chapter 4, we have investigated the structural, magnetic and transport properties of polycrystalline $\mathrm{Sr}_{2-x} \mathrm{Ba}_{x} \mathrm{FeMoO}_{6}$ samples. It was shown that the degree of Anti-Site Disorder (ASD) can be derived using the x-ray diffraction pattern and a single equation obtained from a model using the Powder Cell software. As observed by other investigators, a good correlation between the saturation magnetisation and the ASD was obtained and this was also found in the La-doped samples (chapter 5).

Regarding the magnetic properties, it was found that upon barium substitution the Curie temperature drops systematically from 400 to $315 \mathrm{~K}$. In addition, we have found a large Low Field Magnetoresistance (LFMR) for $x=2$ and shown that there is a correlation between the resistivity and the LFMR. Furthermore, the magnetoresistance can be fitted to the intergrain model of Serrate et al. where the tunneling MR is limited by a disordered region near the insulating grain boundaries. Thus, it is the electronic properties near the grain boundary that lead to the large MR. A model was developed to obtain a better fit in the low field region. High field magnetoresistance measurements were performed and validate our model up to $12 \mathrm{~T}$. In addition, a magnetoresistance as large as $-48 \%$ was measured at $12 \mathrm{~T}$ and $4.2 \mathrm{~K}$, which is close the maximum $-50 \%$ expected in the spin-dependent tunneling magnetoresistance model for polycrystalline samples. At this stage, we do not have any way of controlling the grain boundaries properties and therefore the magnetoresistance. It would be of interest to do further work on the sample preparation technique to optimize the magnetoresistance.

In chapter 5 we studied the effect of the La-substitution on the strontium site, which is expected to be an electronic doping substitution. Using the ASD model presented in chapter 4, it was found that the degree of antisite disorder increases versus the La-concentration from 21.2 to $36.0 \%$ 
for $x=0$ and 0.5 respectively.

The thermoelectric power of $\mathrm{Sr}_{2-x} \mathrm{La}_{x} \mathrm{FeMoO}_{6}$ displays a temperature dependence and shift with electron doping that is remarkably similar to that observed in the superconducting cuprates. We have modelled the Lainduced changes in terms of electronic doping that affects the diffusion and the phonon-drag components.

The decrease in the magnetisation at $6 \mathrm{~T}$ with increasing La-doping was shown to be predominantly due to ASD. The high field magnetisation is extrapolated to be zero at $\backsim 44 \%$ ASD, which is slightly less than the $50 \%$ or completely random Fe-Mo site occupancy. The low temperature magnetisation data was fitted to $m(T)=m_{s}\left(1-a_{0} T^{n}\right)$ and found an $n=1.5$ which is what would be expected for a 3D Heisenberg ferromagnet model. The Curie temperature obtained from the derivative of the temperature dependence of the magnetisation was found to increase versus electron doping from 390 to $415 \mathrm{~K} x=0$ and 0.5 respectively, which supports the band filling scenario.

Finally, it was found that the magnetoresistance in the La-doped samples correlate well with the the degree of antisite disorder. This could imply that the disorder is more likely to occur at the grain boundaries as the transport properties are dominated by spin-dependent tunneling.

In chapter 6, the results from the magnetic and transport measurements on $\mathrm{Sr}_{2} \mathrm{Fe}_{1-x} \mathrm{Al}_{x} \mathrm{MoO}_{6}$ are reported. It was found that the thermoelectric power could be interpreted in terms of electron-doping by the $\mathrm{Al}^{3+}$. This can occur if the Fe valence is below $3+$ as reported from a Mössbauer study on the pure compound.

The Curie temperature decreases with increasing $x$ from 400 to $285 \mathrm{~K}$ for only a $30 \%$ Al-substitution. This can be attributed to a 3D random site dilution such as the 3D Heisenberg or 3D Ising models. Optimising the sample preparation technique to obtain single phase materials with higher $\mathrm{Al}$ concentration is required to determine which of the two models 
fit the experimental data. In addition, theoretical calculations would help to make a rigorous differentiation among the two percolation models.

The saturation moment per f.u., as obtained from magnetisation measurements, is reduced with increasing $x$. The reduction of $m_{s}$ is only 0.39 $\mu_{B} / f . u$. instead of the $0.78 \mu_{B} / f$.u. expected and this is not associated with a change in the ASD as it is nearly constant versus the Al-concentration. The cause of this smaller drop in $m_{s}$ is unknown and would require further research.

The Mo moment, as probed by Mo ZFNMR, shows a small increase of $1.1 \%$ for $x=0.2$. The temperature-dependence of the Mo moment can be fitted to a power law, $1-a_{0} T^{n}$, but $n(1.86)$ is greater than that expected in a 3D Heisenberg model $(n=1.5) . a_{0}$ is found to increase with $x$, which implies a change in the spin-wave dispersion that might be expected from the decrease in $T_{c}$.

While there are systematic changes in the thermoelectric power, magnetisation and ZFNMR data with increasing $x$, it was found that there are no consistent changes in the resistivity or MR at $77 \mathrm{~K}$. It is thus clear that the disorder in the grain boundaries varies non-systematically among the samples. On the other hand, there is a consistent change in the MR at $300 \mathrm{~K}$ and it seems to correlate with the drop of the Curie temperature. A development of the linear behaviour for the larger $x$-concentration shows that the intra-grain contribution becomes more important when the measurement temperature is close to the critical temperature, as observed in other strongly correlated electron systems. This result could be clearly exploited as a linear MR behaviour would be of interest for application purposes.

Results from measurements on a series of oxygen deficient $\mathrm{SrFeO}_{3-\delta}$ samples are reported in chapter 7 . Low field magnetic measurements revealed an increase in the low temperature susceptibility versus the oxygen deficiency which could be interpreted in terms of the DzyaloshinskyMoriya interaction. Spin canting in the $\mathrm{FeO}_{6}$ distorted octahedra would 
be expected to give rise to a ferromagnetic component.

In terms of the magneto-transport measurements, we observed that samples containing mainly the orthorhombic phase exhibit a large magnetoresistance at the antiferromagnetic ordering temperature ( $\sim 240 \mathrm{~K}$ ). The origin of this unusual behaviour is still unclear but further work could be done as large magnetoresistance close to room temperature is of interest for devices.

Temperature dependent measurements of the thermoelectric power were made in $\mathrm{SrFeO}_{3-\delta}$ for the first time. A large drop in the TEP was observed in $\mathrm{SrFeO}_{3-\delta}$ for 3- $\delta=2.865$ to 2.78 at the temperature where the $\mathrm{Sr}_{8} \mathrm{Fe}_{8} \mathrm{O}_{23}$ phase magnetically orders and displays charge-ordering. The drop in the TEP data was attributed to a Verwey-like charge-ordering from the $\mathrm{Sr}_{8} \mathrm{Fe}_{8} \mathrm{O}_{23}$ fraction where $\mathrm{SrFeO}_{3-\delta}$ is an inhomogeneous mixture of $\mathrm{SrFeO}_{3}, \mathrm{Sr}_{8} \mathrm{Fe}_{8} \mathrm{O}_{23}$ and $\mathrm{Sr}_{4} \mathrm{Fe}_{4} \mathrm{O}_{11}$.

Finally, it was shown that the thermoelectric power at room temperature could be used to determine the oxygen content in $\mathrm{SrFeO}_{3-\delta}$. A change in the TEP sign was observed at $50 \% \mathrm{Fe}^{3+}-50 \% \mathrm{Fe}^{4+}$ and this could be explained in terms of a gap in the density of states at the Fermi energy. 


\section{References}

[1] P. A. Cox, Transition Metal Oxides, Clarendon Press - Oxford, 1992.

[2] J. G. Bednorz and K. A. Müller, Z. Phys. B 64, 189 (1986).

[3] J. G. Bednorz and K. A. Müller, Rev. Mod. Phys. 60, 585 (1988).

[4] L. Gao, Y. Y. Xue, F. Chen, Q. Xiong, R. L. Meng, D. Ramirez, C. W. Chu, J. H. Eggert, and H. K. Mao, Phys. Rev. B 50, 4260 (1994).

[5] K. Chahara, T. Ohno, M. Kasai, and Y. Kozono, Appl. Phys. Lett. 63, 1990 (1993).

[6] R. von Helmolt, J. Wecker, B. Holzapfel, L. Schultz, and K. Samwer, Phys. Rev. Lett. 71, 2331 (1993).

[7] T. Miyazaki and N. Tezuka, J. Magn. Magn. Mater. 139, L231 (1995).

[8] J. S. Moodera, L. R. Kinder, T. M. Wong, and R. Meservey, Phys. Rev. Lett. 74, 3273 (1995).

[9] G. A. Prinz, Science 282, 1660 (1998).

[10] G. A. Prinz, J. Magn. Magn. Mater. 200, 57 (1999).

[11] K.-I. Kobayashi, T. Kimura, H. Sawada, K. Terakura, and Y. Tokura, Nature 395, 677 (1998). 
[12] T. Saitoh, M. Nakatake, A. Kakizaki, H. Nakajima, O. Morimoto, S. Xu, Y. Morimoto, N. Hamada, and Y. Aiura, Phys. Rev. B 66, 035112 (2002).

[13] Y. Moritomo, S. Xu, T. Akimoto, A. Machida, N. Hamada, K. Ohoyama, E. Nishibori, M. Takata, and M. Sakata, Phys. Rev. B 62, 14224 (2000).

[14] Y. Moritomo, S. Xu, A. Machida, T. Akimoto, E. Nishibori, M. Takata, and M. Sakata, Phys. Rev. B 61, R7827 (2000).

[15] J. Navarro, C. Frontera, L. Balcells, B. Martínez, and J. Frontcuberta, Phys. Rev. B 64, 092411 (2001).

[16] D. Rubi, C. Frontera, J. Fontcuberta, M. Wojcik, E. Jedryka, and C. Ritter, Phys. Rev. B 70, 094405 (2004).

[17] C. Zener, Phys. Rev. B 82, 440 (1951).

[18] P. W. Anderson and H. Hasegawa, Phys. Rev. B 100, 67 (1955).

[19] D. D. Sarma, Curr. Opin. Solid State Mater. Sci. 5, 261 (2001).

[20] D. D. Sarma, P. Mahadevan, T. Saha-Dasgupta, S. Ray, and A. Kumar, Phys. Rev. Lett. 85, 2549 (2000).

[21] C. Ritter, M. R. Ibarra, L. Morellon, J. Blasco, J. García, and J. M. D. Teresa, J. Phys.: Condens. Matter 12, 8295 (2000).

[22] K.-I. Kobayashi, T. Okuda, Y. Tomioka, T. Kimura, and Y. Tokura, J. Mag. Magn. Mater. 17, 218 (2000).

[23] J. B. Philipp, P. Majewski, L. Alff, A. Erb, R. Gross, T. Graf, M. S. Brandt, J. Simon, T. Whather, W. Mader, D. Topwal, and D. D. Sarma, Phys. Rev. B 68, 144431 (2003). 
[24] A. S. Ogale, S. B. Ogale, R. Ramesh, and T. Venkatesan, Appl. Phys. Lett. 75, 537 (1999).

[25] J. Navarro, J. Nogués, J. S. Muñoz, and J. Fontcuberta, Phys. Rev. B 67, 174416 (2003).

[26] D. Niebieskikwiat, F. Prado, A. Caneiro, and R. D. Sánchez, Phys. Rev. B 70, 132412 (2004).

[27] N. Aschroft and N. Mermin, Solid State Physics, Thomson Learning Inc., 1976.

[28] M. S. Moreno, J. E. Gayone, M. Abbate, A. Caneiro, D. Niebieskikwiat, R. D. Sanchez, A. de Siervo, R. Landers, and G. Zampieri, Solid State Comm. 120, 161 (2001).

[29] J. Lindén, T. Yamamoto, M. Karppinen, H. Yamauchi, and T. Pietari, Appl. Phys. Lett. 76, 2925 (2000).

[30] B. Fisher, K. B. Chashka, L. Patlagan, and G. M. Reisner, Curr. Appl. Phys. 4, 512 (2004).

[31] A. H. Habib, A. Saleem, C. V. Tomy, and D. Bahadur, J. Appl. Phys 97, 10A906 (2005).

[32] J. B. Goodenough, Phys. Rev. B 100, 564 (1955).

[33] C. Gleitzer and J. B. Goodenough, Struct. Bonding 61, 1 (1985).

[34] T. Takeda, Y. Yamaguchi, and H. Watanabe, J. Phys. Soc. Jpn. 33, 967 (1972).

[35] P. K. Gallagher, J. B. MacChesney, and D. N. E. Buchanan, J. Chem. Phys. 41, 2429 (1964).

[36] H. Watanabe, J. Phys. Soc. Jpn. 12, 515 (1957). 
[37] P. Adler, A. Lebon, V. Damljanović, C. Ulrich, C. Bernhard, A. V. Boris, A. Maljuk, C. T. Lin, and B. Keimer, Phys. Rev. B 73, 094451 (2004).

[38] J. B. MacChesney, P. K. Gallagher, and D. N. E. Buchanan, J. Chem. Phys. 43, 1907 (1965).

[39] M. Schmidt, M. Hofmann, and S. J. Campbell, J. Phys.: Condens. Matter 15, 8691 (2003).

[40] A. Lebon, P. Adler, C. Berhard, A. V. Boris, A. V. Pimenov, A. Maljuk, C. T. Lin, C. Ulrich, and B. Keimer, Phys. Rev. Lett. 92, 037202 (2004).

[41] J. P. Hodges, S. Short, J. D. Jorgensen, X. Xiong, B. Dabrowski, S. M. Mini, and C. W. Kimball, J. Solid State Chem. 151, 190 (2000).

[42] S. K. Park, T. Ishikawa, Y. Tokura, J. Q. Li, and Y. Matsui, Phys. Rev. B 60, 10788 (1999).

[43] A. J. M. Kuipers and V. A. M. Brabers, Phys. Rev. B 20, 594 (1979).

[44] A. J. M. Kuipers and V. A. M. Brabers, Phys. Rev. B 14, 1401 (1976).

[45] E. K. Hemery, G. V. M. Williams, and H. Trodahl, Curr. Appl. Phys. 6, 312 (2006).

[46] R. B. Roberts, Philosophical Magazine 36, 91 (1977).

[47] M. McElresh, Fundamentals of magnetism and magnetic measurements, Quantum Design, Inc., 1994.

[48] S. K. Goh, G. V. M. Williams, E. K. Hemery, and H. K. Lee, Phys. Rev. B 74, 134506 (2006).

[49] D. Serrate, J. M. D. Teresa, P. A. Algarabel, M. R. Ibarra, and J. Galibert, Phys. Rev. B 71, 104409 (2005). 
[50] B.-G. Kim, Y.-S. Hor, and S.-W. Cheong, Appl. Phys. Lett. 79, 388 (2001).

[51] E. K. Hemery, G. V. M. Williams, and H. Trodahl, Physica B 390, 175 (2007).

[52] E. K. Hemery, G. V. M. Williams, and H. Trodahl, J. Magn. Magn. Mater. 310, 1958 (2007).

[53] R. Shannon, Acta Crystallogr., Sect. A : Cryst. Phys., Diffr., Theor. Gen. Crystallogr. 32, 751 (1976).

[54] L. Balcells, J. Navarro, M. Bibes, A. Roig, B. Martínez, and J. Frontcuberta, Appl. Phys. Lett. 78, 781 (2001).

[55] J. Navarro, L. Balcells, F. Sandiumenge, M. Bibes, A. Roig, B. Martínez, and J. Fontcuberta, J. Phys.: Condens. Matter 13, 8481 (2001).

[56] A. Arrott and J. E. Noakes, Phys. Rev. Lett. 19, 786 (1967).

[57] A. Maignan, B. Raveau, C. Martin, and C. M. Hervieu, J. Solid State Chem. 144, 224 (1999).

[58] J. Inoue and S. Maekawa, Phys. Rev. B 53, R11 927 (1996).

[59] M. Poz̀ek, A. Dulc̀ić, D. Paar, A. Hamzić, M. Basletić, E. Tafar, G. V. M. Williams, and S. Krämer, Phys. Rev. B 65, 174514 (2002).

[60] B. Fisher, K. B. Chashka, L. Patlagan, and G. M. Reisner, J. Mag. Magn. Mater. 272, 1790 (2004).

[61] J. Navarro, J. Fontcuberta, M. Izquierdo, J. Avila, , and M. C. Asensio, Phys. Rev. B 69, 115101 (2004).

[62] J. L. Alonso, L. A. Fernández, F. Guinea, F. Lesmes, and V. MartínMayor, Phys. Rev. B 67, 214423 (2003). 
[63] D. Serrate, J. M. D. Teresa, J. Blasco, M. R. Ibarra, L. Morellon, and C. Ritter, Eur. Phys. J. B 39, 35 (2004).

[64] D. Rubi, C. Frontera, G. Herranz, J. L. G. Muñoz, and J. Fontcuberta, J. Appl. Phys. 95, 7082 (2004).

[65] M. Wojcik, E. Jedryka, S. Nadolski, J. Navarro, D. Rubi, and J. Fontcuberta, Phys. Rev. B 69, 100407(R) (2004).

[66] J. Lindén, T. Shimada, T. Motohashi, H. Yamauchi, and M. Karppinen, Solid State Commun. 129, 129 (2004).

[67] E. K. Hemery, G. V. M. Williams, and H. Trodahl, Phys. Rev. B 74, 054423 (2006).

[68] Y. Tomioka, T. Okuda, Y. Okimoto, R. Kumai, K.-I. Kobayashi, and T. Tokura, Phys. Rev. B 61, 422 (2000).

[69] D. Niebieskikwiat, A. Caneiro, R. D. Sánchez, and J. Fontcuberta, Phys. Rev. B 64, 180406(R) (2001).

[70] H. J. Trodahl, Phys. Rev. B 51, R6175 (1995).

[71] S. D. Obertelli, J. R. Cooper, and J. L. Tallon, Phys. Rev. B 46, R14928 (1992).

[72] R. D. Barnard, Thermoelectricity in metals and alloys, Taylor and Francis, 1972.

[73] G. C. McIntosh and A. B. Kaiser, Phys. Rev. B 54, 12569 (1996).

[74] T. Saitoh, M. Nakatake, H. Nakajima, O. Morimoto, A. Kakizaki, S. Xu, Y. Moritomo, N. Hamada, and Y. Aiura, Phys. Rev. B 72, 045107 (2005).

[75] V. A. Rowe and P. A. Schroeder, J. Phys. Chem. Solids 31, 1 (1970). 
[76] D. Jiles, Introduction to Magnetism and Magnetic Materials, Chapman and Hall/CRC, 1998.

[77] J. Lindén, T. Yamamoto, J. Nakamura, M. Karppinen, and H. Yamauchi, Appl. Phys. Lett. 78, 2736 (2001).

[78] T. H. Kim, M. Uehara, and S.-W. Cheong, Appl. Phys. Lett. 74, 1737 (1999).

[79] Z. Fang, K. Terakura, and J. Kanamori, Phys. Rev. B 63, 180407(R) (2001).

[80] J. H. Kim, G. Y. Ahn, S.-I. Park, and C. S. Kim, J. Magn. Magn. Mater. 282, 295 (2004).

[81] J. Blasco, C. Ritter, L. Morellon, P. A. Algarabel, J. M. D. Teresa, D. Serrate, J. Garcia, and M. I. Ibarra, Solid State Sci. 4, 651 (2002).

[82] X. M. Feng, G. H. Rao, G. Y. Liu, H. F. Yang, W. F. Liu, Z. W. Ouyang, and J. K. Liang, Physica B 344, 21 (2004).

[83] C. Ritter, J. Blasco, J. M. D. Teresa, D. Serrate, L. Morellon, J. García, and M. R. Ibarra, Solid State Sci. 6, 419 (2004).

[84] A. Arulraj, K. Ramesha, J. Gopalakrishnan, and C. N. R. Rao, J. Solid State Chem. 155, 233 (2000).

[85] E. K. Hemery, G. V. M. Williams, and H. Trodahl, Physica B 394, 74 (2007).

[86] Y. Moritomo, H. Kusuya, A. Machida, E. Nishibori, M. Takata, M. Sakata, and A. Nakamura, J. Phys. Soc. Jpn 70, 3182 (2001).

[87] M. Itoh, I. Ohta, and Y. Inaguma, Mater. Sci. Eng. B 41, 55 (1996).

[88] G. Popov, M. Greenblatt, and M. Croft, Phys. Rev. B 67, 024406 (2003). 
[89] F. Sher, A. Venimadhav, M. G. Blamire, B. Dabrowski, S. Kolesnik, and J. P. Attfield, Solid State Sci. 7, 912 (2005).

[90] A. Peña, J. Gutiérrez, L. M. Rodríguez-Martínez, J. M. Barandiarán, T. Hernández, and T. Rojo, J. Phys. Condens. Matter 13, 6535 (2001).

[91] A. Komoda and A. Pekalski, J. Phys. C: Solid State Phys. 16, 3167 (1983).

[92] S. Kirkpatrick, Rev. Mod. Physics 45, 574 (1973).

[93] Z. Feng and M. S. Seehra, Phys. Rev. B 45, 2184 (1992).

[94] M. Tovar, M. T. Causa, A. Butera, J. Navarro, B. Martínez, J. Fontcuberta, and M. C. G. Passeggi, Phys. Rev. B 66, 024409 (2002).

[95] C. Kapusta, P. C. Riedi, D. Zajac, M. Sikora, J. M. D. Teresa, L. Morellon, and M. R. Ibarra, J. Mag. Magn. Mater. 242, 701 (2002).

[96] O. Chmaissem, R. Kruk, B. Dabrowski, D. E. Brown, X. Xiong, S. Kolesnik, J. D. Jorgensen, and C. W. Kimball, Phys. Rev. B 62, 14197 (2000).

[97] R. Harris, Nuclear Magnetic Resonance Spectroscopy, John Wiley and Sons Inc., 1983.

[98] D. Zajac, C. Kapusta, P. C. Riedi, M. Sikora, C. Oates, D. Rybicki, J. M. D. Teresa, D. Serrate, C. Marquina, and M. R. Ibarra, Acta Physica Polonica A 106, 759 (2004).

[99] Y. M. Zhao, R. Mahendiran, N. Nguyen, B. Raveau, and R. H. Yao, Phys. Rev. B 64, 024414 (2001).

[100] S. Srinath, M. M. Kumar, M. L. Post, and H. Srikanth, Phys. Rev. B 72, 054425 (2006).

[101] E. J. W. Verwey and P. W. Haayman, Physica 8, 979 (1941). 
[102] F. Walz, J. Phys.: Condens. Matter 14, R285 (2002).

[103] M. Jaime, P. Lin, S. H. Chun, M. B. Salamon, P. Dorsey, and M. Rubinstein, Phys. Rev. B 60, 1028 (1999).

[104] E. K. Hemery, G. V. M. Williams, and H. Trodahl, Phys. Rev. B 75, 092403 (2007).

[105] S. Nakamura and S. Iida, Jpn. J. Appl. Phys. 34, 291 (1995).

[106] K. Binder and A. P. Young, Review of Modern Physics 58, 801 (1986).

[107] R. Vidya, P. Ravindran, H. Fjellvàg, and A. Kjekshus, Phys. Rev. B 74, 054422 (2006).

[108] I. Dzyaloshinski, J. Phys. Chem. Solids 4, 241 (1958).

[109] T. Moriya, Phys. Rev. 120, 91 (1960).

[110] D. Bergman and D. Stroud, Solid State Physics, Academic Press, 1992.

[111] N. J. Mott, Proc. Phys. Soc. A. 62, 416 (1949).

[112] W. Koshibae, K. Tsutsui, and S. Maekawa, Phys. Rev. B 62, 6869 (2000).

[113] K. Berggold, M. Kriener, C. Zobel, A. Reichl, M. Reuther, R. Muller, A. Freimuth, and T. Lorenz, Solid State Sci. 72, 155116 (2005).

[114] A. Maignan, D. Flahaut, and S. Herbert, Eur. Phys. J. B 39, 145 (2004).

[115] A. A. Taskin, A. N. Lavrov, and Y. Ando, Phys. Rev. B 73, 121101(R) (2006).

[116] F. Poulsen, G. Lauvstad, and R. Tunold, Solid State Ionics 72, 47 (1994).

[117] P. M. Chaikin and G. Beni, Phys. Rev. B 13, 647 (1976). 


\section{List of Publications}

1. E. K. Hemery, G. V. M. Williams, J. D. Graham and H. J. Trodahl, Reduced Magnetization and Magneto-resistance in $\mathrm{Sr}_{2} \mathrm{Fe}_{1-x} \mathrm{Cr}_{x} \mathrm{MoO}_{6}$ with increasing $\mathrm{Cr}$ substitution, Curr. Appl. Phys. (in press).

2. E. K. Hemery, G. V. M. Williams, and H. J. Trodahl, The effect of isoelectronic substitution on the magneto-resistance of $\mathrm{Sr}_{2-x} \mathrm{Ba}_{x} \mathrm{FeMoO}$, Physica B 390, 175 (2007).

3. E. K. Hemery, G. V. M. Williams, and H. J. Trodahl, Anomalous thermoelectric power in $\mathrm{SrFeO}_{3-\delta}$ from charge-ordering and phase separation, Phys. Rev. B 75, 092403 (2007).

4. E. K. Hemery, G. V. M. Williams, and H. J. Trodahl, Isoelectronic and electronic doping in $\mathrm{Sr}_{2} \mathrm{FeMoO}_{6}$, J. Magn. Magn. Mater. 310, 1958 (2007).

5. E. K. Hemery, G. V. M. Williams, and H. J. Trodahl, The effects of Al substitution on the magnetic and electronic properties of $S r_{2} \mathrm{Fe}_{1-x} A l_{x} \mathrm{MoO}_{6}$, Physica B 394, 74 (2007).

6. E. K. Hemery, G. V. M. Williams, and H. J. Trodahl, $S r_{2} \mathrm{FeMoO}_{6}$ Double perovskites and the effects of the aluminium substitution, Proceedings of the $17^{\text {th }}$ National Congress, Brisbane, Australian Institute of Physics ISBN 09598064-7-4 (2007). 
7. E. K. Hemery, G. V. M. Williams, and H. J. Trodahl, La-induced changes in the magnetic and electronic properties of $\mathrm{Sr}_{2-x} \mathrm{La}_{x} \mathrm{FeMoO}{ }_{6}$, Phys. Rev. B 74, 054423 (2006).

8. E. K. Hemery, G. V. M. Williams, and H. J. Trodahl, The effect of the preparation method and grain morphology on the physical properties of $A_{2} \mathrm{FeMoO}_{6}(A$ $=S r, B a)$, Curr. Appl. Phys. 6, 312 (2006).

9. E. K. Hemery, G. V. M. Williams, and H. J. Trodahl, $\mathrm{Sr}_{2} \mathrm{FeMoO}_{6} \mathrm{Dou}^{-}$ ble perovskites and the effects of Electronic doping by La substitution, Proceedings of the $30^{\text {th }}$ Annual Condensed Matter and Materials Meeting, Wagga Wagga, Australian Institute of Physics ISBN 1-920791-09-4 (2006).

10. S. K. Goh, G. V. M. Williams, E. K. Hemery, and H. K. Lee, Superconducting and magnetic properties of $\mathrm{Ru}_{1-y} \mathrm{M}_{y} \mathrm{Sr}_{2} \mathrm{Eu}_{2-x} \mathrm{Ce} \mathrm{Cu}_{2} \mathrm{O}_{10+\delta} \mathrm{M}=\mathrm{Nb}$,Sn, Phys. Rev. B 74, 134506 (2006).

11. S. K. Goh, E. K. Hemery, G. V. M. Williams, and H. K. Lee, Magnetic and electronic properties of $\mathrm{Ru}_{1-y} \mathrm{M}_{y} \mathrm{Sr}_{2} \mathrm{Eu}_{2-x} \mathrm{Ce}_{x} \mathrm{Cu}_{2} \mathrm{O}_{10+\delta}(\mathrm{M}=\mathrm{Nb}$, Sn), Proceedings of the $30^{\text {th }}$ Annual Condensed Matter and Materials Meeting, Wagga Wagga, Australian Institute of Physics ISBN 1-920791-09-4 (2006). 